\title{
On Keller's Conjecture in Dimension Seven
}

\author{
Andrzej P. Kisielewicz Magdalena Łysakowska \\ Wydział Matematyki, Informatyki i Ekonometrii, Uniwersytet Zielonogórski \\ ul. Z. Szafrana 4a, 65-516 Zielona Góra, Poland \\ \{A.Kisielewicz, M.Lysakowska\}@wmie.uz.zgora.pl
}

Submitted: Mar 5, 2014; Accepted: Dec 30, 2014; Published: Jan 20, 2015

Mathematics Subject Classifications: 05B45, 52C22

\begin{abstract}
A cube tiling of $\mathbb{R}^{d}$ is a family of pairwise disjoint cubes $[0,1)^{d}+T=\left\{[0,1)^{d}+\right.$ $t: t \in T\}$ such that $\bigcup_{t \in T}\left([0,1)^{d}+t\right)=\mathbb{R}^{d}$. Two cubes $[0,1)^{d}+t,[0,1)^{d}+s$ are called a twin pair if $\left|t_{j}-s_{j}\right|=1$ for some $j \in[d]=\{1, \ldots, d\}$ and $t_{i}=s_{i}$ for every $i \in[d] \backslash\{j\}$. In 1930, Keller conjectured that in every cube tiling of $\mathbb{R}^{d}$ there is a twin pair. Keller's conjecture is true for dimensions $d \leqslant 6$ and false for all dimensions $d \geqslant 8$. For $d=7$ the conjecture is still open. Let $x \in \mathbb{R}^{d}, i \in[d]$, and let $L(T, x, i)$ be the set of all $i$ th coordinates $t_{i}$ of vectors $t \in T$ such that $\left([0,1)^{d}+t\right) \cap\left([0,1]^{d}+x\right) \neq \emptyset$ and $t_{i} \leqslant x_{i}$. Let $r^{-}(T)=\min _{x \in \mathbb{R}^{d}} \max _{1 \leqslant i \leqslant d}|L(T, x, i)|$ and $r^{+}(T)=\max _{x \in \mathbb{R}^{d}} \max _{1 \leqslant i \leqslant d}|L(T, x, i)|$. It is known that if $r^{-}(T) \leqslant 2$ or $r^{+}(T) \geqslant 6$, then Keller's conjecture is true for $d=7$. In the present paper we show that it is also true for $d=7$ if $r^{+}(T)=5$. Thus, if $[0,1)^{d}+T$ is a counterexample to Keller's conjecture in dimension seven, then $r^{-}(T), r^{+}(T) \in\{3,4\}$.
\end{abstract}

Key words: box; cube tiling; Keller's conjecture; rigidity.

\section{Introduction}

A cube tiling of $\mathbb{R}^{d}$ is a family of pairwise disjoint cubes $[0,1)^{d}+T=\left\{[0,1)^{d}+t: t \in T\right\}$ such that $\bigcup_{t \in T}\left([0,1)^{d}+t\right)=\mathbb{R}^{d}$. Two cubes $[0,1)^{d}+t,[0,1)^{d}+s$ are called a twin pair if $\left|t_{j}-s_{j}\right|=1$ for some $j \in[d]=\{1, \ldots, d\}$ and $t_{i}=s_{i}$ for every $i \in[d] \backslash\{j\}$. In 1907, Minkowski [18] conjectured that in every lattice cube tiling of $\mathbb{R}^{d}$, that is, when $T$ is a lattice in $\mathbb{R}^{d}$, there is a twin pair, and in 1930, Keller [7] generalized this conjecture to arbitrary cube tiling of $\mathbb{R}^{d}$. Minkowski's conjecture was confirmed by Hajós [6] in 1941 . In 1940, Perron [19] proved that Keller's conjecture is true for all dimensions $d \leqslant 6$ (see also [15]).

In 1992, Lagarias and Shor [12], using ideas from Corrádi's and Szabó's papers [3, 20], constructed a cube tiling of $\mathbb{R}^{10}$ which does not contain a twin pair and thereby refuted Keller's cube tiling conjecture. In 2002, Mackey [17] gave a counterexample to Keller's 
conjecture in dimension eight, which also shows that this conjecture is false in dimension nine. For $d=7$ Keller's conjecture is still open.

Let $[0,1)^{d}+T$ be a cube tiling, $x \in \mathbb{R}^{d}$ and $i \in[d]$, and let $L(T, x, i)$ be the set of all $i$ th coordinates $t_{i}$ of vectors $t \in T$ such that $\left([0,1)^{d}+t\right) \cap\left([0,1]^{d}+x\right) \neq \emptyset$ and $t_{i} \leqslant x_{i}$ (Figure 1 ). For every $x \in \mathbb{R}^{d}$ and $i \in[d]$ the set $L(T, x, i)$ contains at most $2^{d-1}$ elements.

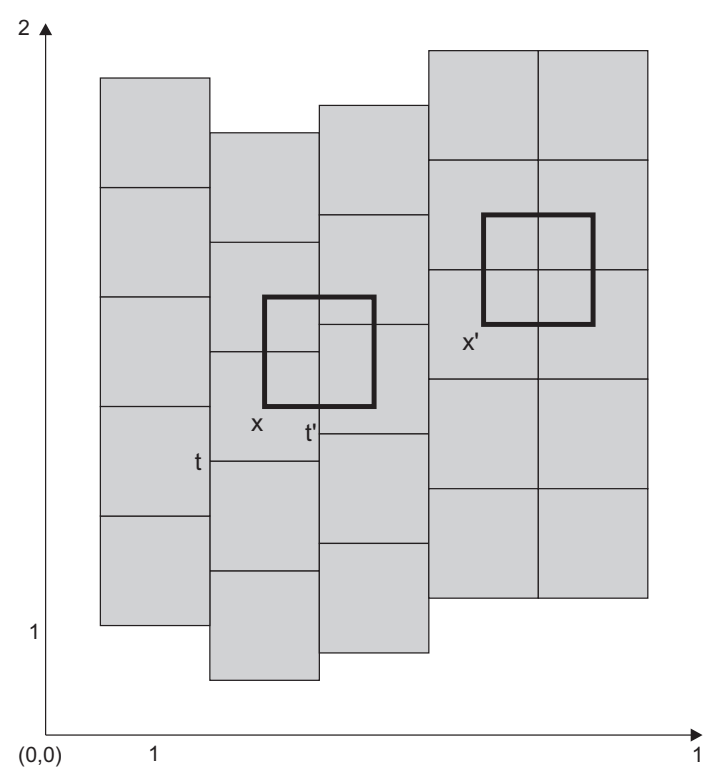

Figure 1: A portion of a cube tiling $[0,1)^{2}+T$ of $\mathbb{R}^{2}$. The number of elements in $L(T, x, i)$ depends on the position of $x \in \mathbb{R}^{2}$. For $x=(2,3)$, we have $L(T, x, 1)=\{3 / 2\}\left(=\left\{t_{1}\right\}\right)$ and $L(T, x, 2)=\{5 / 2,11 / 4\}\left(=\left\{t_{2}, t_{2}^{\prime}\right\}\right)$, while for $x^{\prime}=(4,15 / 4)$, we have $L\left(T, x^{\prime}, 1\right)=\{7 / 2\}$ and $L\left(T, x^{\prime}, 2\right)=\{13 / 4\}$. This portion of the tiling $[0,1)^{2}+T$ shows that $r^{-}(T)=1$ and $r^{+}(T)=2$.

Let

$$
r^{-}(T)=\min _{x \in \mathbb{R}^{d}} \max _{1 \leqslant i \leqslant d}|L(T, x, i)| \quad \text { and } \quad r^{+}(T)=\max _{x \in \mathbb{R}^{d}} \max _{1 \leqslant i \leqslant d}|L(T, x, i)| .
$$

In 2010, Debroni et al. [4] computed, using the supercomputer Cray XT5 Kraken, that Keller's conjecture is true for all cube tilings $[0,1)^{7}+T$ of $\mathbb{R}^{7}$ such that $T \subset(1 / 2) \mathbb{Z}^{7}$. This result shows that Keller's conjecture is true for cube tilings of $\mathbb{R}^{7}$ with $r^{-}(T) \leqslant 2$ ([10]). In [10] we showed that Keller's conjecture is true for cube tilings $[0,1)^{7}+T$ of $\mathbb{R}^{7}$ for which $r^{+}(T) \geqslant 6$. In this paper we prove that

Theorem 1. Keller's conjecture is true for all cube tilings $[0,1)^{7}+T$ of $\mathbb{R}^{7}$ for which $r^{+}(T)=5$.

It follows from the above results that if $[0,1)^{7}+T$ is a counterexample to Keller's conjecture in dimension seven, then $r^{-}(T), r^{+}(T) \in\{3,4\}$ (Corollary 30). 
Keller's cube tiling conjecture was not as clearly motivated as Minkowski's conjecture was. Recall that, the existence of a twin pair in a lattice tiling $[0,1)^{d}+T$ determines the form of a basis for the lattice $T$. Keller's conjecture was rather a generalization of Minkowski's conjecture. In our opinion the paper of Lagarias and Shor [13] presents the problem of the existence of twin pairs in cube tilings of $\mathbb{R}^{d}$ in an appropriate manner. In this important work, roughly speaking, the authors measure distances between some of the cubes in a tiling $[0,1)^{d}+T$. When the dimension of the space increases, the distances between cubes can also increase. In [13] Lagarias and Shor gave an estimation of how fast the distances between cubes increase. A twin pair is a pair of cubes with the minimal possible distance in a cube tiling. It follows from Perron's result that for $d \leqslant 6$ in an arbitrary cube tiling of $\mathbb{R}^{d}$ there are cubes which are closed (twin pairs). From Mackey's example [17] it is known that in dimension eight the process of cubes moving away in cube tilings has started. Resolving Keller's conjecture for $d=7$ will answer the question whether this process had already begun in dimension seven.

Working on Keller's conjecture has, on the one hand, provided the opportunity of answering an old query in tiling theory, and on the other hand is the beginning of a much deeper and more interesting investigation into the structure of cube tilings of $\mathbb{R}^{d}$ in the spirit of Lagarias's and Shor's ideas contained the paper [13]. These investigations, besides describing the structure of tilings, can provide new ideas in topics related to cube tilings. For example, in [9] we showed how a cube tiling code designed in [13] can be used to obtain an interesting partitions and matchings of a $d$-dimensional cube, and in [16] a surprising structure of cube tilings of $\mathbb{R}^{3}$ is described (see also [8]). Moreover, the computations made by David Applegate (see Section 1 in [4]) prove that there is a twin pair in every cube tiling $[0,1)^{6}+T$ of $\mathbb{R}^{6}$ with $r^{-}(T) \leqslant 2$. On the other hand, Theorem 5.2 in [10] shows that there is a twin pair in every cube tiling $[0,1)^{6}+T$ of $\mathbb{R}^{6}$ such that $r^{+}(T) \geqslant 3$. Thus, these two results give a new proof of Keller's conjecture in dimensions $d \leqslant 6([10$, Theorem 5.4]).

To make the paper self-contained we have collected the basic notions in Section 2. We use a very abstract language, in the form of systems of abstract words, but in the long run such an approach simplifies the reasoning. Therefore, Section 2 is very expanded. It contains numerous examples and figures. We belive that it will help the reader in understanding the basic concepts contained in the paper (Section 2 in the presented paper is almost the same as Section 2 in [10].)

The proof of Theorem 1 is based on a structural result dealing with two systems of abstract words having 12 words each (Theorem 27). It can be interpreted by means of systems of cubes in the flat torus $\mathbb{T}^{d}=\left\{\left(x_{1}, \ldots, x_{d}\right)(\bmod 2):\left(x_{1}, \ldots, x_{d}\right) \in \mathbb{R}^{d}\right\}$ as follows: A set $F \subset \mathbb{T}^{d}$ is called a polycube if $F$ has a tiling by translates of the unit cube, that is, there is a family of pairwise disjoint translates of the unit cube $\mathscr{F}=[0,1)^{d}+T, T \subset \mathbb{T}^{d}$, such that $\bigcup_{t \in T}[0,1)^{d}+t=F$. The question of how many tilings the polycube $\mathrm{F}$ has is a basic in tiling theory. As we show in Sections 2 and 6 the case $r^{+}(T)=5$ is reduced to the following task: For $d \in\{4,5,6\}$ determine all polycubes $F \subset \mathbb{T}^{d}$ which have at least two twin pair free cube tilings $\mathscr{F}$ and $\mathscr{G}$ such that $\mathscr{F} \cap \mathscr{G}=\emptyset$ and $|\mathscr{F}|=|\mathscr{G}|=12$. The case $r^{+}(T) \geqslant 6$ resolved in [10] relies on showing that no two different twin pair free cube 
tilings exist for a polycube $F$ with 11 cubes or less.

In graph theory knowing the structure of small graphs (graphs with a few vertices) plays an important role. Similarly in cube tilings it is very useful to know the structures of all tilings of polycubes with a few cubes. The essential in the paper small systems of abstract words, which describe the structures of small polycubes, are given in Section 3. In Section 4 we establish necessary conditions that have to be fulfilled by the above mentioned two systems of words. The results from this section allow us to make reductions in the computations which are described in Section 5. The reductions are necessary to make the computations in a reasonable time as the number of all cases that would have to be considered by the computer program is more than $\left(\begin{array}{l}64 \\ 12\end{array}\right) 3^{72}$. At the end of Section 5 based on the results of the computations we give the forms of two systems of abstract words which describe the structures of two disjoint twin pair free cube tilings of a polycube $F$ with 12 cubes (Theorem 27). Finally, in Section 6 using Theorem 27 we prove Theorem 1.

\section{Basic notions}

In this section we present the basic notions on dichotomous boxes and words (details can be found in $[5,11])$. We start with systems of boxes.

In the whole paper, if $\mathscr{X}$ is a family of sets, then $\bigcup \mathscr{X}=\bigcup_{A \in \mathscr{X}} A$. Moreover, if $Y$ is a set, then a partition of $Y$ is a family $\mathscr{Y}$ of its pairwise disjoint subsets such that $\bigcup \mathscr{Y}=Y$.

\subsection{Dichotomous boxes and polyboxes}

Let $X_{1}, \ldots, X_{d}$ be non-empty sets with $\left|X_{i}\right| \geqslant 2$ for every $i \in[d]$. The set $X=X_{1} \times \cdots \times X_{d}$ is called a $d$-box. A non-empty set $K \subseteq X$ is called a box if $K=K_{1} \times \cdots \times K_{d}$ and $K_{i} \subseteq X_{i}$ for each $i \in[d]$. By $\operatorname{Box}(X)$ we denote the set of all boxes in $X$.

The box $K$ is said to be proper if $K_{i} \neq X_{i}$ for each $i \in[d]$ (Figure 2).
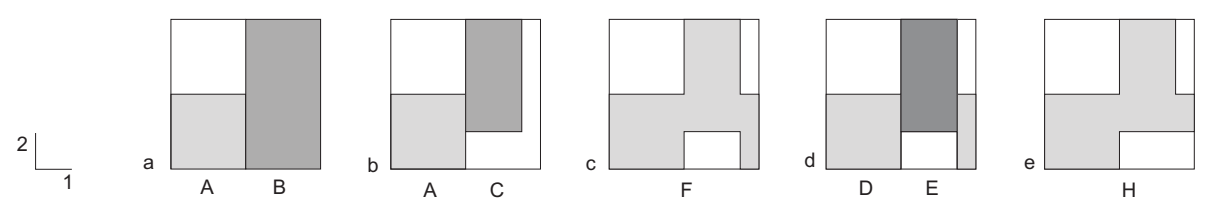

Figure 2: The box $A \subset[0,1]^{2}$ is proper, and $B$ is not. The boxes $A$ and $B$ are dichotomous, while $A$ and $C$ are not. The set $F \subset[0,1]^{2}$ is a polybox, and $\mathscr{F}=\{D, E\}$ is suit for it. Moreover, $F$ is rigid. The set $H$ is not a polybox.

Two boxes $K$ and $G$ in $X$ are called dichotomous if there is $i \in[d]$ such that $K_{i}=$ $X_{i} \backslash G_{i}$. A suit is any collection of pairwise dichotomous boxes. A suit is proper if it consists of proper boxes. A non-empty set $F \subseteq X$ is said to be a polybox if there is a suit $\mathscr{F}$ for $F$, that is, if $\bigcup \mathscr{F}=F$. In other words, $F$ is a polybox if it has a partition into pairwise dichotomous boxes. A polybox $F$ is rigid if it has exactly one suit, that is, if $\mathscr{F}$ 
and $\mathscr{G}$ are suits for a rigid polybox, then $\mathscr{F}=\mathscr{G}$. (Figures $2 c$ and $3 d, e$; the polyboxes $\bigcup \mathscr{F}^{3, B}$ and $\bigcup \mathscr{F}^{3, B^{c}}$ in Figure 5 are not rigid.)
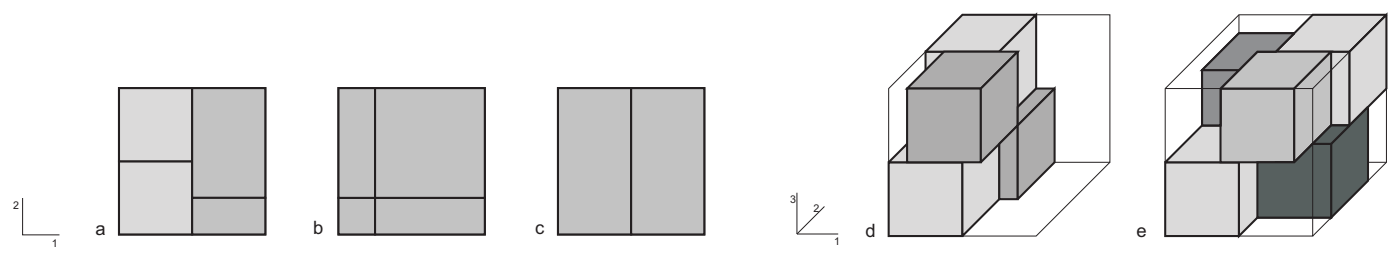

Figure 3: Partition $a$ is a minimal partition of $[0,1]^{2}$, and Partition $b$ is a minimal partition which is at the same time a simple partition. Partition $c$ is a simple partition which is not a minimal partition. The systems of boxes $d, e$ are suits for rigid polyboxes in the 3-box $[0,1]^{3}$.

The important property of proper suits is that, for every proper suits $\mathscr{F}$ and $\mathscr{G}$ for a polybox $F$, we have $|\mathscr{F}|=|\mathscr{G}|$ (see the suits $\mathscr{F}^{3, B}$ and $\mathscr{F}^{3, B^{c}}$ in Figure 5). Thus, we can define a box number $|F|_{0}=$ the number of boxes in any proper suit for the polybox $F$ (compare (2.4) and Theorem 2.4 in [11]). In Figure 5 we have $\left|\bigcup \mathscr{F}^{3, B}\right|_{0}=3$. Obviously, the above property is not true for suits which are not proper (compare suits in Figure $3 a$ and $3 c$ ). A proper suit for a $d$-box $X$ is called a minimal partition of $X$ (Figures 3 and $5)$. In [5] we showed that a suit $\mathscr{F}$ is a minimal partition of a $d$-box $X$ if and only if $|\mathscr{F}|=2^{d}$.

A family $\mathscr{C} \subset \operatorname{Box}(X)$ is called a simple partition of $X$ if for every $K, G \in \mathscr{C}$ and every $i \in[d]$ we have $K_{i}=G_{i}$ or, if $G_{i} \neq X_{i}, K_{i}=X_{i} \backslash G_{i}$ and $\mathscr{C}$ is a suit for $X$ (Figures $3 b$ and $3 c$ ).

Two boxes $K, G \subset X$ are said to be a twin pair if $K_{j}=X_{j} \backslash G_{j}$ for some $j \in[d]$ and $K_{i}=G_{i}$ for every $i \in[d] \backslash\{j\}$. Alternatively, two dichotomous boxes $K, G$ are a twin pair if $K \cup G$ is a box. (The suits in Figures $3 a, b, c$ contains twin pairs, while the suits in Figures $3 d$, e do not contain a twin pair). Observe that the suit for a rigid polybox cannot contain a twin pair.

\subsection{The structure of minimal partitions}

In order to sketch our approach to the problem of the existence of twin pairs in a cube tiling of $\mathbb{R}^{d}$ we describe the structure of a minimal partition. A graph-theoretic description of this structure can be found in $[2,14]$ (see also [13]).

Let $X$ be a $d$-box. A set $l_{i}=\left\{x_{1}\right\} \times \cdots \times\left\{x_{i-1}\right\} \times X_{i} \times\left\{x_{i+1}\right\} \times \cdots \times\left\{x_{d}\right\}$, where $x_{j} \in X_{j}$ for $j \in[d] \backslash\{i\}$, is called a line in $X$. A set $F \subseteq X$ is called an $i$-cylinder (Figure 4) if for every line $l_{i}$ one has $l_{i} \cap F=l_{i}$ or $l_{i} \cap F=\emptyset$.

Let $\mathscr{F}$ be a minimal partition, and let $B \subset X_{i}$ be a proper subset of $X_{i}$ set such that there is a box $K \in \mathscr{F}$ with $K_{i} \in\left\{B, B^{c}\right\}$, where $B^{c}=X_{i} \backslash B$. Let

$$
\mathscr{F}^{i, B}=\left\{K \in \mathscr{F}: K_{i}=B\right\} \quad \text { and } \quad \mathscr{F}^{i, B^{c}}=\left\{K \in \mathscr{F}: K_{i}=B^{c}\right\} .
$$




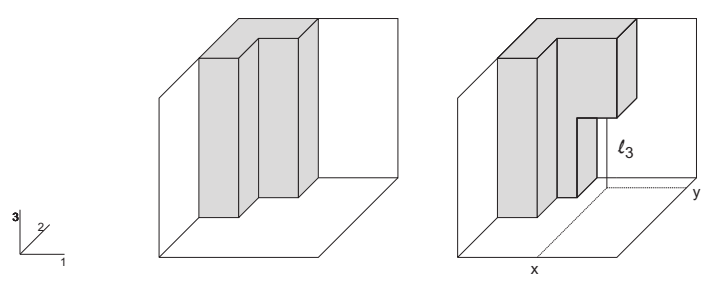

Figure 4: The set on the left is a 3-cylinder in $X=[0,1]^{3}$, and the set on the right is not because the line $l_{3}=\{x\} \times\{y\} \times[0,1]$ has a non-empty intersection with this set but $l_{3}$ is not entire contained in it.

Since boxes in $\mathscr{F}$ are pairwise dichotomous, the set $\bigcup\left(\mathscr{F}^{i, B} \cup \mathscr{F}^{i, B^{c}}\right)$ is an $i$-cylinder, and the set of boxes $\mathscr{F}^{i, B} \cup \mathscr{F}^{i, B^{c}}$ is a suit for it (Figure 5). The partition $\mathscr{F}$ is minimal, and therefore $|\mathscr{F}|=2^{d}$. Thus, the boxes in $\mathscr{F}$ can form at most $2^{d-1}$ pairwise disjoint $i$-cylinders. More precisely, for every $i \in[d]$ there are sets $B^{1}, \ldots, B^{k_{i}} \subset X_{i}$ such that $B^{n} \notin\left\{B^{m},\left(B^{m}\right)^{c}\right\}$ for every $n, m \in\left[k_{i}\right], n \neq m$, and

$$
\mathscr{F}=\mathscr{F}^{i, B^{1}} \cup \mathscr{F}^{i,\left(B^{1}\right)^{c}} \cup \cdots \cup \mathscr{F}^{i, B^{k_{i}}} \cup \mathscr{F}^{i,\left(B^{k_{i}}\right)^{c}} .
$$

The boxes in $\mathscr{F}$ are proper, and hence $\left|\mathscr{F}^{i, B^{n}} \cup \mathscr{F}^{i,\left(B^{n}\right)^{c}}\right| \geqslant 2$ for every $n \in\left[k_{i}\right]$. Thus, $k_{i} \leqslant 2^{d-1}$ for every $i \in[d]$. (That is why $1 \leqslant|L(T, x, i)| \leqslant 2^{d-1}$ for every cube tiling $[0,1)^{d}+T, x \in \mathbb{R}^{d}$ and $\left.i \in[d].\right)$

If $K$ is a box in $X$ and $\mathscr{G}$ is a family of boxes, then let

$$
K_{i^{c}}=K_{1} \times \cdots \times K_{i-1} \times K_{i+1} \times \cdots \times K_{d} \quad \text { and } \quad \mathscr{G}_{i^{c}}=\left\{K_{i^{c}}: K \in \mathscr{G}\right\} .
$$

(In [11] the set $K_{i^{c}}$ is denoted by $K_{i^{\prime}}$.) Moreover, let $x_{i^{c}}=\left(x_{1}, \ldots, x_{i-1}, x_{i+1}, \ldots, x_{d}\right)$ for $x \in X$.

Since $\bigcup\left(\mathscr{F}^{i, B} \cup \mathscr{F}^{i, B^{c}}\right)$ is an $i$-cylinder, we have $\bigcup \mathscr{F}^{i, B}=\bigcup \mathscr{F}^{i c}{ }^{i, B^{c}}$. Moreover, the sets of boxes $\mathscr{F}_{i^{c}}^{i, B}=\left(\mathscr{F}^{i, B}\right)_{i^{c}}$ and $\mathscr{F}_{i^{c}}^{i, B^{c}}=\left(\mathscr{F}^{i, B^{c}}\right)_{i^{c}}$ are two suits for the polybox $\bigcup \mathscr{F}_{i^{c}}^{i, B}$, which is a polybox in the $(d-1)$-box $X_{i^{c}}$ (Figure 5). Note that as $\mathscr{F}_{i^{c}}^{i, B}$ and $\mathscr{F}_{i^{c}}^{i, B^{c}}$ are proper suits for the polybox $\bigcup \mathscr{F}_{i^{c}}^{i, B}$, we have $\left|\mathscr{F}_{i^{c}}^{i, B}\right|=\left|\mathscr{F}_{i^{c}}^{i, B^{c}}\right|$.

If now $K, G \in \mathscr{F}$ is a twin pair, then there is a suit $\mathscr{F}^{i, B} \cup \mathscr{F}^{i, B^{c}} \subset \mathscr{F}$ for some $i$-cylinder such that $K, G \in \mathscr{F}^{i, B} \cup \mathscr{F}^{i, B^{c}}$. Thus, $K, G \in \mathscr{F}^{i, B}$ or $K, G \in \mathscr{F}^{i, B^{c}}$ or $K \in \mathscr{F}^{i, B}$ and $G \in \mathscr{F}^{i, B^{c}}$. In the third case $K_{i^{c}} \in \mathscr{F}_{i^{c}}^{i, B} \cap \mathscr{F}_{i^{c}}^{i, B^{c}}$, where $K_{i^{c}}=G_{i^{c}}$. So, if $\mathscr{F}_{i^{c}}^{i, B} \cap \mathscr{F}_{i^{c}}^{i, B^{c}} \neq \emptyset$, then there is a twin pair in $\mathscr{F}$. (In $[10$, Section 2.2] we present a comparison of the structure of a minimal partition and a graph-theoretic description of this structure given in $[2,14]$.)

\subsection{Cube tilings and dichotomous boxes}

Every two cubes $[0,1)^{d}+t$ and $[0,1)^{d}+p$ in an arbitrary cube tiling $[0,1)^{d}+T$ of $\mathbb{R}^{d}$ satisfy Keller's condition: There is $i \in[d]$ such that $t_{i}-p_{i} \in \mathbb{Z} \backslash\{0\}$ ([7]). For any cube $[0,1]^{d}+x$, where $x \in \mathbb{R}^{d}$, the family of boxes $\mathscr{F}_{x}=\left\{\left([0,1)^{d}+t\right) \cap\left([0,1]^{d}+x\right) \neq\right.$ 


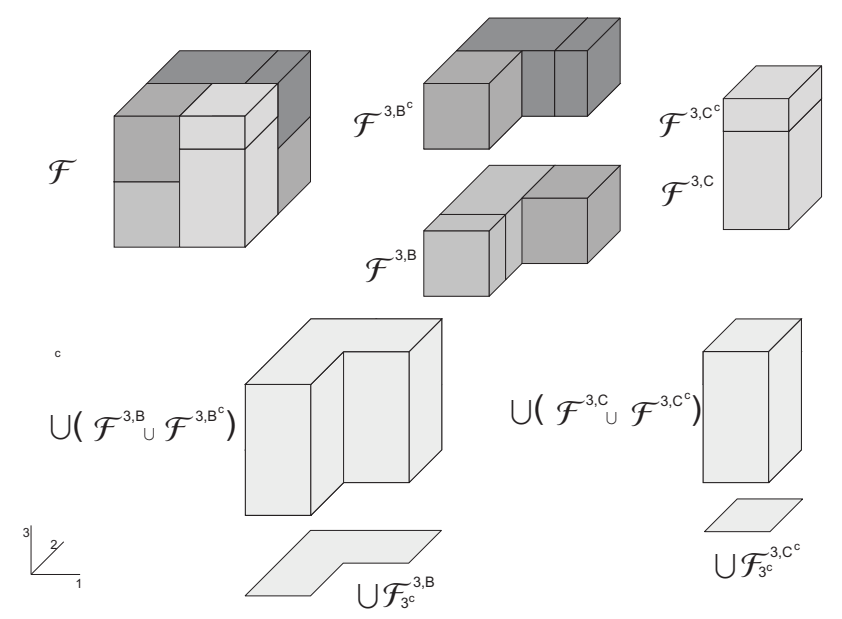

Figure 5: The minimal partition $\mathscr{F}=\mathscr{F}^{3, B} \cup \mathscr{F}^{3, B^{c}} \cup \mathscr{F}^{3, C} \cup \mathscr{F}^{3, C^{c}}$ of the 3 -box $X=[0,1]^{3}$ $(B=[0,1 / 2), C=[0,3 / 4))$. The set $\bigcup\left(\mathscr{F}^{3, B} \cup \mathscr{F}^{3, B^{c}}\right)$ is a 3 -cylinder and $\mathscr{F}^{3, B} \cup \mathscr{F}^{3, B^{c}}$ is a proper suit for it.

$\emptyset: t \in T\}$ is a partition of the cube $[0,1]^{d}+x$ in which, because of Keller's condition, every two boxes $K, G \in \mathscr{F}_{x}$ are dichotomous: There is $i \in[d]$ such that $K_{i}$ and $G_{i}$ are disjoint and $K_{i} \cup G_{i}=[0,1]+x_{i}$. Moreover, since cubes in cube tilings are half-open, every box in $\mathscr{F}_{x}$ is proper, and consequently the family $\mathscr{F}_{x}$ is a minimal partition. The structure of the partition $\mathscr{F}_{x}$ reflects the local structure of the cube tiling $[0,1)^{d}+T$. Obviously, a cube tiling $[0,1)^{d}+T$ contains a twin pair if and only if the partition $\mathscr{F}_{x}$ contains a twin pair for some $x \in \mathbb{R}^{d}([14,19])$ (see Figure 1). Observe also that if $\mathscr{F}_{x}=\mathscr{F}_{x}^{i, B^{1}} \cup \mathscr{F}_{x}^{i,\left(B^{1}\right)^{c}} \cup \cdots \cup \mathscr{F}_{x}^{i, B^{k_{i}(x)}} \cup \mathscr{F}_{x}^{i,\left(B^{k_{i}(x)}\right)^{c}}$, then $|L(T, x, i)|=k_{i}(x)$ (compare $(2.1))$.

The idea to consider the cubes $[0,1)^{d}+t, t \in T$, for which $\left([0,1)^{d}+t\right) \cap\left([0,1]^{d}+x\right) \neq \emptyset$ comes from Perron [19].

\subsection{Key result in proving Theorem 1}

Let $[0,1)^{7}+T$ be a cube tiling of $\mathbb{R}^{7}$, and let $\mathscr{F}_{x}$ be as defined in the previous section. If $r^{+}(T)=5$, then

$$
\mathscr{F}_{x}=\mathscr{F}^{i, B^{1}} \cup \mathscr{F}^{i,\left(B^{1}\right)^{c}} \cup \cdots \cup \mathscr{F}^{i, B^{5}} \cup \mathscr{F}^{i,\left(B^{5}\right)^{c}} .
$$

for some $x \in \mathbb{R}^{7}$ and some $i \in[7]$.

Assume that there are no twin pairs in the tiling $[0,1)^{7}+T$. Then $\mathscr{F}_{x}$ does not contain a twin pair. It follows from [10, Corollary 4.6] (compare Theorem 15 in Section 3) that $\left|\mathscr{F}^{i, B^{n}}\right| \geqslant 12$ for every $n \in[5]$. Thus, there is at least one $n \in[5]$ such that $\left|\mathscr{F}^{i, B^{n}}\right|=12$ because $\left|\mathscr{F}_{x}\right|=128$ and $\left|\mathscr{F}^{i, B^{j}}\right|=\left|\mathscr{F}^{i,\left(B^{j}\right)^{c}}\right|$ for every $j \in[5]$. The suits $\mathscr{F}_{i^{c}}^{i, B^{n}}$ and 
$\mathscr{F}_{i^{c}}^{i,\left(B^{n}\right)^{c}}$ are disjoint and do not contain a twin pair (see the last part of Section 2.2).

The main effort in the paper will be rely on describing the forms of all disjoint and twin pair free suits $\mathscr{F}_{i^{c}}^{i, B^{n}}$ and $\mathscr{F}_{i^{c}}^{i,\left(B^{n}\right)^{c}}$ such that $\left|\mathscr{F}_{i^{c}}^{i, B^{n}}\right|=\left|\mathscr{F}_{i^{c}}^{i,\left(B^{n}\right)^{c}}\right|=12$ and $\bigcup \mathscr{F}_{i^{c}}^{i, B^{n}}=$ $\bigcup \mathscr{F}_{i^{c}}^{i,\left(B^{n}\right)^{c}}$. In Theorem 27, which is our key result in proving Theorem 1, we give the forms of the suits $\mathscr{F}_{i^{c}}^{i, B^{n}}$ and $\mathscr{F}_{i^{c}}^{i,\left(B^{n}\right)^{c}}$. Having this, we will prove Theorem 1 quite easily.

\subsection{Dichotomous words and polybox codes}

The results in the present paper are formulated and proved in full generality. Suits have the form of systems of abstract words. We collect below basic notions concerning words (details can be found in [11]).

A set $S$ of arbitrary objects will be called an alphabet, and the elements of $S$ will be called letters. A permutation $s \mapsto s^{\prime}$ of the alphabet $S$ such that $s^{\prime \prime}=\left(s^{\prime}\right)^{\prime}=s$ and $s^{\prime} \neq s$ is said to be a complementation. Each sequence of letters $s_{1} \ldots s_{d}$ from the set $S$ is called a word. The set of all words of length $d$ is denoted by $S^{d}$. Two words $u=u_{1} \ldots u_{d}$ and $v=v_{1} \ldots v_{d}$ are dichotomous if there is $j \in[d]$ such that $u_{j}^{\prime}=v_{j}$. If $V \subseteq S^{d}$ consists of pairwise dichotomous words, then we call it a polybox code (or polybox genome). (In the next section we give examples of polybox codes and their relationships with suits.) Two words $u, v \in S^{d}$ form a twin pair if there is $j \in[d]$ such that $u_{j}^{\prime}=v_{j}$ and $u_{i}=v_{i}$ for every $i \in[d] \backslash\{j\}$.

Let $A=\left\{i_{1}<\cdots<i_{n}\right\} \subseteq[d]$ and $A^{c}=[d] \backslash A$. Then $u_{A}=u_{i_{1}} \ldots u_{i_{n}}$ and $V_{A}=\left\{v_{A}\right.$ : $v \in V\}$ for $V \subseteq S^{d}$. If $i \in[d]$ and $A=\{i\}^{c}$, then we write $u_{i^{c}}$ and $V_{i^{c}}$ instead of $u_{\{i\}^{c}}$ and $V_{\{i\}^{c}}$, respectively. If $V \subseteq S^{d}, l \in S$ and $i \in[d]$, then $V^{i, l}=\left\{v \in V: v_{i}=l\right\}$. The representation

$$
V=V^{i, l_{1}} \cup V^{i, l_{1}^{\prime}} \cup \cdots \cup V^{i, l_{k_{i}}} \cup V^{i, l_{k_{i}}^{\prime}},
$$

where $l_{j}, l_{j}^{\prime} \in S$ and $V^{i, l_{j}} \cup V^{i, l_{j}^{\prime}} \neq \emptyset$ for $j \in\left[k_{i}\right]$, will be called a distribution of words in $V$. For every $l \in S$ and $i \in[d]$ let $V_{i^{c}}^{i, l}=\left(V^{i, l}\right)_{i^{c}}$.

Let us discuss briefly a connection between dichotomous words and adjacent vertices in a d-dimensional Keller graph ([3]), which is a graph on the vertex set $\{0,1,2,3\}^{d}$ in which two vertices $v, w \in\{0,1,2,3\}^{d}$ are adjacent if there are $i, j \in[d], i \neq j$, such that $v_{i} \neq w_{i}, v_{j} \neq w_{j}$ and $\left|v_{i}-w_{i}\right|=2$ or $\left|v_{j}-w_{j}\right|=2$. Define a complementation on the alphabet $\{0,1,2,3\}$ by $0^{\prime}=2$ and $1^{\prime}=3$. Thus, two vertices $v, w \in\{0,1,2,3\}^{d}$ are adjacent in the Keller graph if and only if the words $v, w$ are dichotomous and they do not form a twin pair. In the paper we consider polybox codes $V$ whose words are written down in an alphabet $S$ which has more then four letters and therefore the elements of $V$ cannot be considered as vertices of the Keller graph. But when $V \subset\left\{a, a^{\prime}, b, b^{\prime}\right\}^{d}$, the reader who is familiar with the Keller graphs may assume that $0=a, 2=a^{\prime}, 1=b$ and $3=b^{\prime}$.

\subsection{Realizations of polybox codes}

Let $X=X_{1} \times \cdots \times X_{d}$ be a $d$-box. Suppose that for each $i \in[d]$ a mapping $f_{i}: S \rightarrow$ $\operatorname{Box}\left(X_{i}\right) \backslash\left\{X_{i}\right\}$ is such that $f_{i}\left(s^{\prime}\right)=X_{i} \backslash f_{i}(s)$ for $s \in S$. We define the mapping 
$f: S^{d} \rightarrow \operatorname{Box}(X)$ by

$$
f\left(s_{1} \ldots s_{d}\right)=f_{1}\left(s_{1}\right) \times \cdots \times f_{d}\left(s_{d}\right) .
$$

About such defined $f$ we will say that it preserves dichotomies. If $V \subseteq S^{d}$, then the set of boxes $f(V)=\{f(v): v \in V\}$ is said to be a realization of the set of words $V$. Figures 6 and 7 present realizations of polybox codes in various $d$-boxes $X$ (see also Example 4).

Clearly, if $V$ is a polybox code, then $f(V)$ is a suit for the polybox $\bigcup f(V)$. The realization is said to be exact if for each pair of words $v, w \in V$, if $v_{i} \notin\left\{w_{i}, w_{i}^{\prime}\right\}$, then $f_{i}\left(v_{i}\right) \notin\left\{f_{i}\left(w_{i}\right), X_{i} \backslash f_{i}\left(w_{i}\right)\right\}$ (Figure 6).

A polybox code $V \subseteq S^{d}$ is called a partition code if any realization $f(V)$ of $V$ is a suit for a $d$-box $X$. For example, $V=\left\{a a, a a^{\prime}, a^{\prime} b, a^{\prime} b^{\prime}\right\}$ is a partition code (Figure 6), while the polybox codes $V, W$ whose realizations are presented in Figure 7 are not partition codes. Observe that, if $V \subseteq S^{d}$ is a partition code, then $f(V)$ is a minimal partition. Indeed, for every $v \in V$ the box $f(v)$ is proper and thus $f(V)$ is a proper suit for $X$. Moreover, if a partition code $V \subseteq S^{d}$ has a distribution of words of the form (2.2), and $\mathscr{F}$ is an exact realization of $V$, then for every $j \in\left[k_{i}\right]$ the set $\mathscr{F}^{i, B^{j}} \cup \mathscr{F}^{i,\left(B^{j}\right)^{c}}$ is an exact realization of the polybox code $V^{i, l_{j}} \cup V^{i, l_{j}^{\prime}}$, where $\mathscr{F}^{i, B^{j}} \cup \mathscr{F}^{i,\left(B^{j}\right)^{c}}$, for $j \in\left[k_{i}\right]$, are as in (2.1).

A partition code $C \subseteq S^{d}$ is said to be simple if for every $v, w \in C$ and every $i \in[d]$ we have $v_{i}=w_{i}$ or $v_{i}=w_{i}^{\prime}$. For example, $U=\left\{a b, a^{\prime} b, a b^{\prime}, a^{\prime} b^{\prime}\right\}$, where $a, b \in S$, is a simple partition code, while $V=\left\{a a, a a^{\prime}, a^{\prime} b, a^{\prime} b^{\prime}\right\}$ is not a simple partition code.

We will exploit some abstract but very useful realization of polybox codes. This sort of realization was invented in [1], where it was the crucial tool in proving the main theorem of that paper (compare [11, Section 2]).

Let $S$ be an alphabet with a complementation, and let

$$
\begin{gathered}
E S=\left\{B \subset S:\left|\left\{s, s^{\prime}\right\} \cap B\right|=1, \text { whenever } s \in S\right\}, \\
E s=\{B \in E S: s \in B\}
\end{gathered}
$$

Let $V \subseteq S^{d}$ be a polybox code, and let $v \in V$. The equicomplementary realization of the word $v$ is the box

$$
\breve{v}=E v_{1} \times \cdots \times E v_{d}
$$

in the $d$-box $(E S)^{d}=E S \times \cdots \times E S$. The equicomplementary realization of the code $V$ is the family

$$
E(V)=\{\breve{v}: v \in V\} .
$$

If $S$ is finite, $s_{1}, \ldots, s_{n} \in S$ and $s_{i} \notin\left\{s_{j}, s_{j}^{\prime}\right\}$ for every $i \neq j$, then

$$
\left|E s_{1} \cap \cdots \cap E s_{n}\right|=\left(1 / 2^{n}\right)|E S| .
$$

In the paper we will assume that $S$ is finite, unless it will be explicitly stated otherwise.

The value of the realization $E(V)$, where $V \subseteq S^{d}$, lies in the equality (2.3) (which does not hold for translates of the unit interval $[0,1)$ in $\left.\mathbb{T}^{1}\right)$. In particular, boxes in $E(V)$ are of the same size: $\left|E v_{i}\right|=(1 / 2)|E S|$ for every $i \in[d]$ and consequently $|\breve{v}|=\left(1 / 2^{d}\right)|E S|^{d}$ 
for $v \in E(V)$. Thus, two boxes $\breve{v}, \breve{w} \subset(E S)^{d}$ are dichotomous if and only if $\breve{v} \cap \breve{w}=\emptyset$. The same is true for cubes in a cube tiling of a polycube $F \subset \mathbb{T}^{d}$ and therefore working with the boxes $\breve{v}, v \in V$, we can think of them as translates of the unit cube in $\mathbb{T}^{d}$.

Moreover, from (2.3) we obtain the following important lemma ([10]).

Lemma 2. Let $w, u, v \in S^{d}$, and let $\mathscr{D}$ be a simple partition of the $d$-box $\breve{w}$. If boxes $\breve{w} \cap \breve{u}$ and $\breve{w} \cap \breve{v}$ belong to $\mathscr{D}$, then there is a simple partition code $C \subseteq S^{d}$ such that $u, v \in C$. In particular, if $\breve{w} \cap \breve{u}$ and $\breve{w} \cap \breve{v}$ form a twin pair, then $u$ and $v$ are a twin pair.

In a general case the above lemma is not true; for a given three boxes $K, G$ and $H$ in a $d$-box $X$ such that $K$ and $G$ are dichotomous and $K \cap H, G \cap H$ belong to a simple partition of $H$, it can happen that $K$ and $G$ are not members of the same simple partition of $X$. For example, let $X=[0,4] \times[0,4], K=[2,4] \times[2,4], G=[2,3) \times[0,2)$ and $H=[1,3) \times[1,3)$. Then the boxes $K \cap H=[2,3) \times[2,3), G \cap H=[2,3) \times[1,2)$ belong to a simple partition $\mathscr{C}=\{[1,2) \times[1,2),[1,2) \times[2,3),[2,3) \times[1,2),[2,3) \times[2,3)\}$ of $H$. But $K$ and $G$ do not belong to the same simple partition of $X$ because $K_{1} \notin\left\{G_{1}, X_{1} \backslash G_{1}\right\}$.

Let $V \subseteq S^{d}$ be a polybox code, and $f(V)$ an exact realization of $V$. The set $f(V)$ is a suit (a set of pairwise dichotomous boxes), while $V$ describes the structure of it. The code $V$ has infinitely many exact realizations which may be very different from each other. For example, the partitions in Figures $6 a, b, c$ are pairwise different but they are all the exact realizations of the polybox code $V=\left\{a a, a a^{\prime}, a^{\prime} b, a^{\prime} b^{\prime}\right\}$. The differences can even be related to the number of dimensions of a specific partition; the sets in Partition $6 c$ are 3-dimensional, but this partition can be regarded as 2-dimensional minimal partition with the same structure as Partitions $6 a$ and $6 b$.

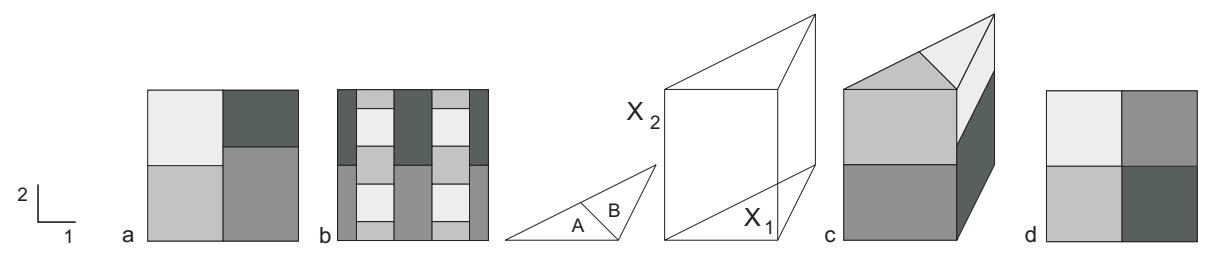

Figure 6: Partitions $a-c$ are exact realization of the code $V=\left\{a a, a a^{\prime}, a^{\prime} b, a^{\prime} b^{\prime}\right\}$. Partition $d$ is also a realization of $V$ but not exact. Partition $c$ is a minimal partition of the 2-box $X=X_{1} \times X_{2}$, where $X_{1}$ is the triangle $A \cup B$, and $X_{2}=[0,1]$. The realizations $a, b, d$ are partitions of 2-box $[0,1]^{2}$; in the first realization we have $f_{1}(a)=f_{2}(a)=[0,1 / 2)$ and $f_{2}(b)=[0,5 / 8)$; in the second one we have $f_{1}(a)=[1 / 8,3 / 8) \cup(5 / 8,7 / 8), f_{2}(a)=[0,1 / 2)$ and $f_{2}(b)=[1 / 8,3 / 8) \cup(5 / 8,7 / 8)$; in the third case $f_{1}(a)=A$ (and then $f_{1}\left(a^{\prime}\right)=B$ ), $f_{2}(a)=[0,1 / 2)$ and $f_{2}(b)=[0,5 / 8)$; in the last partition we have $f_{i}(a)=f_{i}(b)=[0,1 / 2)$ for $i=1,2$.

\subsection{Equivalent and rigid polybox codes}

Let $V, W \subseteq S^{d}$ be polybox codes, and let $v \in S^{d}$. We say that $v$ is covered by $W$, and write $v \sqsubseteq W$, if $f(v) \subseteq \bigcup f(W)$ for every mapping $f$ that preserves dichotomies. 
For example, the word $v=b b$ is not covered by the code $W=\left\{a a, a^{\prime} b, a^{\prime} b^{\prime}\right\}$ because there is a realization $f(W)$ such that $f(b b) \not \subset \bigcup f(W)$. For example, if $X=[0,1]^{2}$, $f_{1}(a)=f_{2}(a)=[0,1 / 2)$ and $f_{1}(b)=[1 / 3,2 / 3), f_{2}(b)=[0,2 / 3)$, then $f(b b)=[1 / 3,2 / 3) \times$ $[0,2 / 3) \not \subset[0,1 / 2)^{2} \cup[1 / 2,1] \times[0,1]$ (obviously, one can find a realization $g(W)$ such that $g(v) \subset \bigcup g(W))$. It can be easily checked that for every $l \in S$ the word $w=l a$ is covered by $W$ (see also Example 4 ).

If $v \sqsubseteq W$ for every $v \in V$, then we write $V \sqsubseteq W$.

Polybox codes $V, W \subseteq S^{d}$ are said to be equivalent if $V \sqsubseteq W$ and $W \sqsubseteq V$ (Figure 7). Thus, $V$ and $W$ are equivalent if and only if $\bigcup f(V)=\bigcup f(W)$ for every mapping $f$ that preserves dichotomies. Obviously, if $V$ and $W$ are equivalent, then $|V|=|W|$ (see also comments below (2.6)).

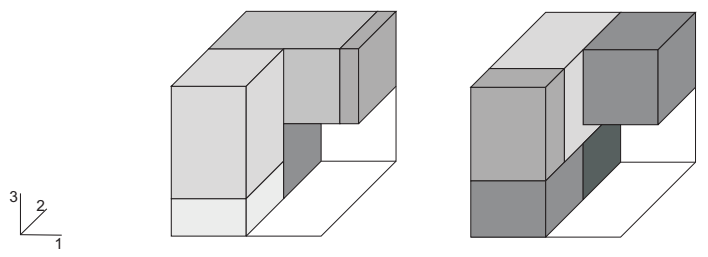

Figure 7: Realizations $f(V), f(W)$ in the 3-box $X=[0,1]^{3}$ of equivalent polybox codes $V, W \subset\left\{a, a^{\prime}, b, b^{\prime}, c, c^{\prime}\right\}^{3}$, where $V=\left\{a a a, a a a^{\prime}, a a^{\prime} b, b a^{\prime} b^{\prime}, b^{\prime} a^{\prime} b^{\prime}\right\}$ (on the left) and $W=$ $\left\{a a c, a a^{\prime} c, a b c^{\prime}, a b^{\prime} c^{\prime}, a^{\prime} a^{\prime} b^{\prime}\right\}$ (on the right). In this example we have $f_{1}(a)=[0,1 / 2)$, $f_{1}(b)=[0,7 / 8), f_{2}(a)=[0,1 / 2), f_{2}(b)=[0,1 / 4), f_{3}(a)=[0,1 / 4), f_{3}(b)=[0,1 / 2)$ and $f_{3}(c)=[0,3 / 8)$.

A polybox code $V \subset S^{d}$ is called rigid if there is no code $W \subset S^{d}$ which is equivalent to $V$ and $V \neq W$ (Example 3). Thus, if polybox codes $V, W$ are equivalent and one of them is rigid, then $V=W$. Observe that, rigid polybox codes cannot contain a twin pair.

The following result describes one of the most important property of polybox codes ([11, Theorem 10.6]).

If $w \in S^{d}$ and $V \subseteq S^{d}$ is a polybox code such that $w \sqsubseteq V, w \notin V$, then there is a simple partition code $C \subseteq S^{d}$ and there are two words $v, u \in V \cap C$ such that

$$
\left|\left\{i \in[d]: v_{i}=u_{i}^{\prime}\right\}\right| \equiv 1 \quad(\bmod 2) .
$$

(see Examples 3 and 4). Observe that it follows from the above that if a polybox codes $V \subseteq S^{d}$ is not rigid (which in particular means that $w \sqsubseteq V$ and $w \notin V$ for some $w \in S^{d}$ ), then $V$ has to contain the words $v$ and $u$ such as in (2.4). This property is a starting point in the computations in Section 5.

The definition of the relation $\sqsubseteq$ is rather cumbersome tool to decide whether $w \sqsubseteq V$. Below we give very useful and easy test, especially in the computations, to check whether $w \sqsubseteq V([11$, Theorem 10.4]). 
Let $g: S^{d} \times S^{d} \rightarrow \mathbb{Z}$ be defined by the formula

$$
g(v, w)=\prod_{i=1}^{d}\left(2\left[v_{i}=w_{i}\right]+\left[w_{i} \notin\left\{v_{i}, v_{i}^{\prime}\right\}\right]\right),
$$

where $[p]=1$ if the sentence $p$ is true and $[p]=0$ if it is false.

Let $w \in S^{d}$, and let $V \subseteq S^{d}$ be a polybox code. Then

$$
\breve{w} \subseteq \bigcup E(V) \Leftrightarrow w \sqsubseteq V \Leftrightarrow \sum_{v \in V} g(v, w)=2^{d} .
$$

It follows from the definition of equivalent polybox codes $V, W \subseteq S^{d}$ and (2.6) that $V$ and $W$ are equivalent if and only if $\bigcup E(V)=\bigcup E(W)$. Another characterization of equivalent polybox codes $V, W \subseteq S^{d}$ which stems from (2.6) is the following: Polybox codes $V, W \subseteq S^{d}$ are equivalent if and only if $\sum_{v \in V} g(v, w)=2^{d}$ for every $w \in W$ and $\sum_{w \in W} g(w, v)=2^{d}$ for every $v \in V$.

Example 3. Let $V=\left\{a a a a, a^{\prime} a^{\prime} a^{\prime} a, b a a^{\prime} a, a^{\prime} b a a, a a^{\prime} b a, b b b a^{\prime}\right\}$. If $l \notin\left\{a, a^{\prime}\right\}$, then $\sum_{v \in V} g(v, b b b l)=1+1+2+2+2+8=2^{4}$, and if $l=a$, then $\sum_{v \in V} g(v, b b b l)=$ $2+2+4+4+4+0=2^{4}$. Therefore, for every $l \in S$, by (2.6), bbbl $\sqsubseteq V$. (Thus, $V$ has to contain two words $v, u$ described in (2.4). These are aaaa and $a^{\prime} a^{\prime} a^{\prime} a$.) In particular, for every $l \in S$ the twin pair $b b b l, b b b l^{\prime}$ is covered by $V$. Obviously, again by (2.6), this means that for every realization $f(V)$ the boxes $f(b b b l)$ and $f\left(b b b l^{\prime}\right)$ are contained in $\bigcup f(V)$. It can be checked that $\sum_{v \in V} g(v, w)<2^{d}$ for every $w \in S^{d}$ such that $w \notin \bigcup_{l \in S}\left\{b b b l, b b b l^{\prime}\right\}$. Thus, every such word $w$ is not covered by $V$. Occasionally, we will denote this fact by $w \nsubseteq V$. Moreover, it follows from the above that $V$ is rigid.

Let $X=X_{1} \times \cdots \times X_{d}$ be a $d$-box. For every $i \in[d]$ let $S_{i}$ be the set of all pairs $(A, i)$, where $A$ is a proper subset of $X_{i}$. With the set of all proper boxes in $X$ we associate the set of words $S^{d}$, where $S=\bigcup_{i=1}^{d} S_{i}$. We define a complementation $(A, i) \mapsto(A, i)^{\prime}$ on $S$ by the formula $(A, i)^{\prime}=\left(A^{c}, i\right)$, where $A^{c}=X_{i} \backslash A$. Obviously, if $X_{i}$ is infinite for some $i \in[d]$, then $S$ is infinite.

Observe now that if $\mathscr{F}$ is a proper suit for a polybox $F \subseteq X$, then the set of words $V=\left\{\bar{A}_{1} \ldots \bar{A}_{d}: A_{1} \times \cdots \times A_{d} \in \mathscr{F}\right\} \subseteq S^{d}$, where $\bar{A}_{i}=\left(A_{i}, i\right)$ for $i \in[d]$ is a polybox code, and the suit $\mathscr{F}$ is an exact realization of $V$. (Of course, this is one of the many ways of receiving polybox codes for suits.)

\subsection{Geometry of dichotomous boxes}

In this section we describe the main techniques used in the paper. They are based on the properties of the realization $E(V)$.

Usually we will consider two disjoint sets $V, W \subset S^{d}$ which form equivalent polybox codes. Recall, that polybox codes $V$ and $W$ are equivalent if and only if $\bigcup E(V)=$ $\bigcup E(W)$, where $\bigcup E(V)=\bigcup_{\breve{v} \in E(V)} \breve{v}$ (compare the comment below (2.6)). Moreover, let us recall that if $i \in[d]$ and $v \in S^{d}$, then $v_{i}$ denotes the letter standing in the word $v$ at 
the $i$ th position, while $v_{i^{c}} \in S^{d-1}$ is the word that arises from $v$ by skipping the letter $v_{i}$ in the word $v$. Moreover, $V_{i^{c}}=\left\{v_{i^{c}}: v \in V\right\}$.

Our goal is to reveal the structure of $V$ and $W$ or estimate the cardinality of $V$ (since $V, W$ are equivalent, we have $|V|=|W|$ ). Below we describe the most important techniques applied in the paper.

- The structure of $V$ from the suit for $\breve{w}$. Let $w \sqsubseteq V$. Then $\breve{w} \subseteq \cup E(V)$ and the set of boxes $\mathscr{F}=\{\breve{w} \cap \breve{v}: v \in V\}$ is a suit for $\breve{w}$. In Example 4 we show what kind of information can be obtained from the structure of $\mathscr{F}$.

Example 4. In Figure 8 the five boxes on the left are a realization of the polybox code $V=\left\{a a a, a^{\prime} a^{\prime} a^{\prime}, b a a^{\prime}, a^{\prime} b a, a a^{\prime} b\right\}$, and the box in the middle is a realization of the word $w=b b b$. Since $w \sqsubseteq V$, we have $\breve{w} \subset \bigcup E(V)$. Thus, the 3-box $\breve{w}$ is divided into pairwise dichotomous boxes of the form $\breve{w} \cap \breve{v}$ for $v \in V$, and the set $\bigcup(\{\breve{w} \cap \breve{v}: v \in Q\} \cup\{\breve{w} \cap \breve{v}$ : $v \in P\}$ ), where $P=\left\{v \in V^{3, a}: \breve{w} \cap \breve{v} \neq \emptyset\right\}$ and $Q=\left\{v \in V^{3, a^{\prime}}: \breve{w} \cap \breve{v} \neq \emptyset\right\}$, is a 3-cylinder in the box $\breve{w}$. Therefore, $\bigcup\left\{(\breve{w} \cap \breve{v})_{3^{c}}: v \in Q\right\}=\bigcup\left\{(\breve{w} \cap \breve{v})_{3^{c}}: v \in P\right\}$. Thus, the polybox $\bigcup\left\{(\breve{w} \cap \breve{v})_{3^{c}}: v \in Q\right\}$ is divided twice into pairwise dichotomous boxes without twin pairs and $|Q|=|P|=2$. In Lemma 14 we will show that these two information allow us to predict the form of $Q_{3^{c}}$ and $P_{3^{c}}: Q_{3^{c}}=\left\{b a, a^{\prime} a^{\prime}\right\}$ and $P_{3^{c}}=\left\{a a, a^{\prime} b\right\}$.

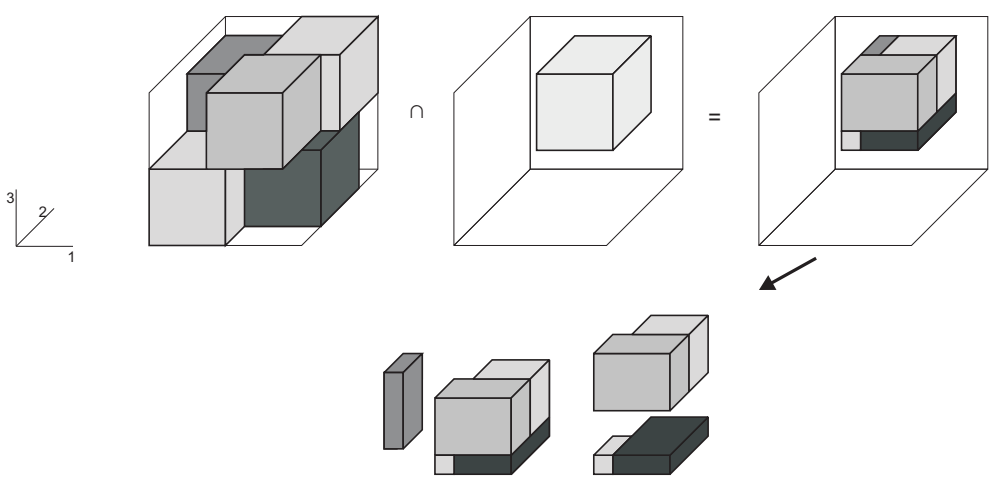

Figure 8: On the top: The light box (in the middle) is contained in the sum of five pairwise dichotomous boxes (the boxes on the left). These boxes determine a partition of the light box into pairwise dichotomous boxes (the partition on the right). On the bottom: The boxes in this partition are arranged into 3-cylinders.

- The structure of $W$ from the distribution of words in $V$. Below, in $(\mathbf{P}),(\mathbf{V}),(\mathbf{C})$ and $(\mathbf{C o})$ we show how to use an information on a distribution of words in $V$ of the form (2.2) to say something about the distribution of words in $W$.

Let $V, W, U \subseteq S^{d}$ be polybox codes and assume that $V$ and $W$ are equivalent. Recall that $V_{i^{c}}^{i, l}=\left(V^{i, l}\right)_{i^{c}}$ for every $i \in[d]$ and $l \in S$.

$(\mathbf{P})$ : Projections. Suppose that there is $x \in \bigcup E\left(V^{i, l^{\prime}}\right)$ such that $x_{i^{c}} \notin \bigcup E\left(V_{i^{c}}^{i, l}\right)$ (see Figure $9 \mathrm{~A}$, where $l=a$ ). Since $\bigcup E(V)=\bigcup E(W)$ and (2.3), the point $x$ can be covered 


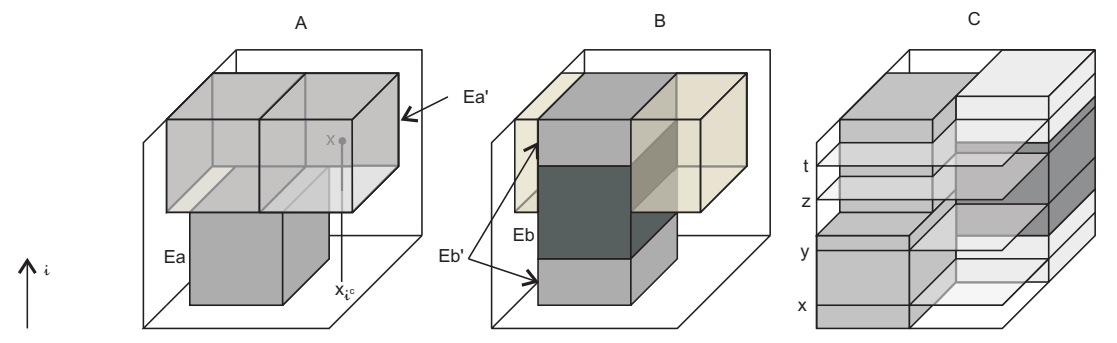

Figure 9: Figure 9: A scheme of realizations $E(V)(\mathrm{A}), E(W)(\mathrm{B})$ and $E(U)(\mathrm{C})$, where $V=V^{i, a} \cup V^{i, a^{\prime}}, W=W^{i, a^{\prime}} \cup W^{i, b} \cup W^{i, b^{\prime}}$ and $U=U^{i, a} \cup U^{i, a^{\prime}} \cup U^{i, b} \cup U^{i, b^{\prime}}$. We assume that the codes $V$ and $W$ are equivalent, and thus $\bigcup E(V)=\bigcup E(W)$.

only by a box $\breve{w} \in E(W)$ such that $w_{i}=l^{\prime}$. Thus, $W^{i, l^{\prime}} \neq \emptyset$. In particular, if $W^{i, l}=\emptyset$ and $W^{i, l^{\prime}} \neq \emptyset$, then $W^{i, l^{\prime}} \sqsubseteq V^{i, l^{\prime}}$ (compare Figures 9A and 9B).

$(\mathbf{S})$ : Slices. By (2.3), for every $r \in E l \cap E s, l \notin\left\{s, s^{\prime}\right\}$ the set $\pi_{r}^{i}=E S \times \cdots \times E S \times$ $\{r\} \times E S \times \cdots \times E S$, where $\{r\}$ stands at the $i$ th position, slices the sets $\bigcup E\left(U^{i, l}\right)$ and $\bigcup E\left(U^{i, s}\right)$ simultaneously (Figure 9C, where $r \in\{x, y, z, t\}$ ).

$(\mathbf{V})$ : Volumes. Let $\left|V^{i, l}\right|=n$ and $\left|V^{i, l^{\prime}}\right|=m$, and let $n<m$. Since all boxes $\breve{u}, u \in S^{d}$, are of the same size and $n<m$, by $(\mathbf{P}),\left|W^{i, l^{\prime}}\right| \geqslant m-n$ (compare Figures 9A and 9B).

(C): Cylinders. Suppose that $V^{i, l} \cup V^{i, l^{\prime}}=\emptyset$ and $W^{i, l} \cup W^{i, l^{\prime}} \neq \emptyset$ for some $l \in S$. Then $\bigcup E\left(W_{i^{c}}^{i, l}\right)=\bigcup E\left(W_{i^{c}}^{i, l^{\prime}}\right)$, and hence the set $\bigcup E\left(W^{i, l} \cup W^{i, l^{\prime}}\right)$ in an $i$-cylinder in the $d$-box $(E S)^{d}$ (compare Figures $9 \mathrm{~A}$ and $9 \mathrm{~B}$, where $\left.l=b\right)$. Indeed, if the set $\bigcup E\left(W^{i, l} \cup W^{i, l^{\prime}}\right)$ is not an $i$-cylinder, then $\bigcup E\left(W_{i^{c}}^{i, l}\right) \neq \bigcup E\left(W_{i^{c}}^{i, l^{\prime}}\right)$. By $(\mathbf{P}), V^{i, l} \cup V^{i, l^{\prime}} \neq \emptyset$, which is not true. Observe that, by (2.6), the codes $W_{i^{c}}^{i, l}$ and $W_{i c}^{i, l^{\prime}}$ are equivalent.

(Co): Covers. Suppose that polybox codes $V_{i^{c}}^{i, l}$ and $W_{i^{c}}^{i, l} \cup W_{i^{c}}^{i, s_{1}} \cup \cdots \cup W_{i^{c}}^{i, s_{k}}$ are equivalent, where $s_{n} \notin\left\{l, l^{\prime}, s_{j}, s_{j}^{\prime}\right\}$ for every $n, j \in[k], n \neq j$. Then

$$
W_{i^{c}}^{i, s_{1}} \cup \cdots \cup W_{i^{c}}^{i, s_{k}} \sqsubseteq V_{i^{c}}^{i, l^{\prime}} \quad \text { and } \quad V_{i^{c}}^{i, l} \sqsubseteq W_{i^{c}}^{i, l} \cup W_{i^{c}}^{i, s_{1}^{\prime}} \cup \cdots \cup W_{i^{c}}^{i, s_{k}^{\prime}} .
$$

(In Figures 9A and 9B the codes $V_{i^{c}}^{i, a}$ and $W_{i^{c}}^{i, a} \cup W_{i^{c}}^{i, b}$ are equivalent, where $a=l, b=s_{1}$, and $\left.W^{i, a}=\emptyset\right)$. Indeed, since boxes in $E(V)$ are pairwise dichotomous and, by (2.6), $\breve{w}_{i^{c}} \subseteq \cup E\left(V_{i^{c}}^{i, l}\right)$ for every $w \in W^{i, s_{1}} \cup \cdots \cup W^{i, s_{k}}$, it follows that each point $x \in \breve{w} \backslash \bigcup E\left(V^{i, l}\right)$ has to be covered by the set $\bigcup E\left(V^{i, l^{\prime}}\right)$. Therefore, $\breve{w}_{i^{c}} \subseteq \bigcup E\left(V_{i c}^{i, l^{\prime}}\right)$ for every $w \in$ $W^{i, s_{1}} \cup \cdots \cup W^{i, s_{k}}$, and consequently, by (2.6), $w_{i^{c}} \sqsubseteq V_{i^{c}}^{i, l^{\prime}}$ for $w \in W^{i, s_{1}} \cup \cdots \cup W^{i, s_{k}}$. Thus, $W_{i c}^{i, s_{1}} \cup \cdots \cup W_{i c}^{i, s_{k}} \sqsubseteq V_{i^{c}}^{i, l^{\prime}}$. In the similar manner we show that $V_{i^{c}}^{i, l} \sqsubseteq W_{i c^{c}}^{i, l} \cup W_{i^{c}}^{i, s_{1}^{\prime}} \cup$ $\cdots \cup W_{i^{c}}^{i, s_{k}^{\prime}}$.

For fixed $x \in E S$ and $i \in[d]$ let

$$
\pi_{x}^{i}=E S \times \cdots \times E S \times\{x\} \times E S \times \cdots \times E S,
$$

where $\{x\}$ stands at the $i$ th position. 
- The structure of $V$ and $W$ from slices of the sets $\bigcup E(V)$ and $\bigcup E(W)$ by a set $\pi_{x}^{i}$. Let $V, W \subseteq S^{d}$ be equivalent polybox codes. Then, by (2.6), $\bigcup E(V)=\bigcup E(W)$, and therefore $\pi_{x}^{i} \cap \bigcup E(V)=\pi_{x}^{i} \cap \bigcup E(W)$ for every $x \in E S$. In the next lemma we show that this last equality implies that the codes $\left\{v_{i^{c}}: v \in V\right.$ and $\left.\pi_{x}^{i} \cap \breve{v} \neq \emptyset\right\}$ and $\left\{w_{i^{c}}: w \in W\right.$ and $\left.\pi_{x}^{i} \cap \breve{w} \neq \emptyset\right\}$ are equivalent. Moreover, we will pay attention whether these codes are rigid (Figure 10) because their rigidity can help to estimate the number of words in $V$ and $W$.

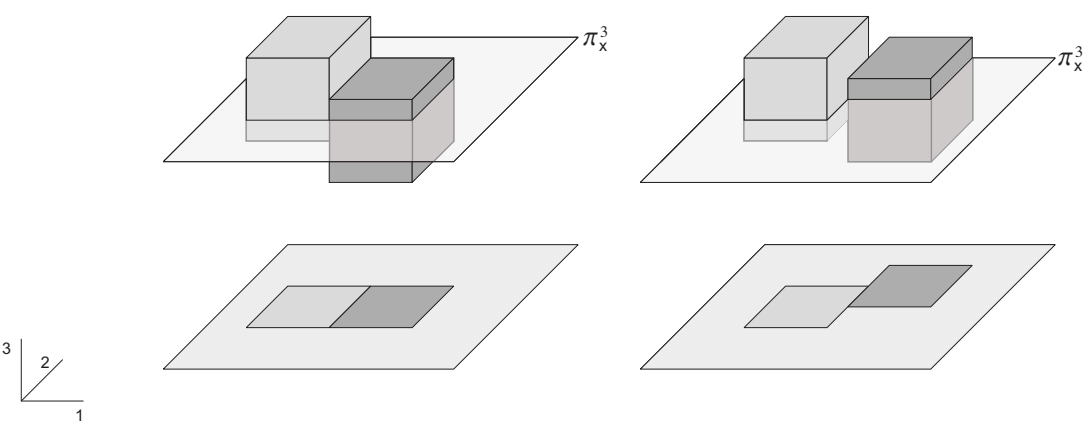

Figure 10: The realizations $f(V)$ and $f(U)$ of two polybox codes $V=\{v, u\}$, where $v=a a a$ (the light box) and $u=a^{\prime} a b$ (boxes on the left) and $U=\{v, w\}$, where $w=a^{\prime} b b$ (boxes on the right) in the 3-box $X=[0,1]^{3}$ ( $X$ is not pictured). The polyboxes $\bigcup f(V)$ and $\bigcup f(U)$ are sliced by the set $\pi_{x}^{3}=[0,1]^{2} \times\{x\}$, where $x \in f_{3}(a) \cap f_{3}(b)$. Below each slice we have polyboxes in $X_{i^{c}}=[0,1]^{2}$ for $i=3$, which are realizations of the codes $\left\{v_{i^{c}}, u_{i^{c}}\right\}$ (on the left) and $\left\{v_{i^{c}}, w_{i^{c}}\right\}$ (on the right). The polybox code $\left\{v_{i^{c}}, w_{i^{c}}\right\}$ is rigid, while $\left\{v_{i^{c}}, u_{i^{c}}\right\}$ is not because it is a twin pair.

Lemma 5. Let $S=\left\{l_{1}, l_{1}^{\prime}, \ldots, l_{m}, l_{m}^{\prime}\right\}, \varepsilon \in\{0,1\}^{m}$ and $A_{\varepsilon}=\left\{l_{1}^{\varepsilon_{1}}, \ldots, l_{m}^{\varepsilon_{m}}\right\}$, where $l_{k}^{0}=l_{k}$ and $l_{k}^{1}=l_{k}^{\prime}$ for $k \in[m]$. If $V, W \subseteq S^{d}$ are equivalent polybox codes, then for every $i \in[d]$ and $\varepsilon \in\{0,1\}^{m}$ the polybox codes

$$
\bigcup_{l \in A_{\varepsilon}} V_{i^{c}}^{i, l} \text { and } \bigcup_{l \in A_{\varepsilon}} W_{i^{c}}^{i, l}
$$

are equivalent.

Proof. Since the polybox codes $V$ and $W$ are equivalent, by (2.6), $\bigcup E(V)=\bigcup E(W)$. Thus, $\pi_{x}^{i} \cap \bigcup E(V)=\pi_{x}^{i} \cap \bigcup E(W)$ for every $i \in[d]$ and $x \in E S$. By (2.3), the set $\bigcap_{l \in A_{\varepsilon}} E l$ is non-empty. Let $z \in \bigcap_{l \in A_{\varepsilon}} E l$. Since $\pi_{z}^{i} \cap \bigcup E(V)=\bigcup\left\{\pi_{z}^{i} \cap \breve{v}: v \in \bigcup_{l \in A_{\varepsilon}} V^{i, l}\right\}$ and $\pi_{z}^{i} \cap$ $\bigcup E(W)=\bigcup\left\{\pi_{z}^{i} \cap \breve{w}: w \in \bigcup_{l \in A_{\varepsilon}} W^{i, l}\right\}$, it follows that $\bigcup E\left(\bigcup_{l \in A_{\varepsilon}} V_{i^{c}}^{i, l}\right)=\bigcup E\left(\bigcup_{l \in A_{\varepsilon}} W_{i^{c}}^{i, l}\right)$. By (2.6), the codes $\bigcup_{l \in A_{\varepsilon}} V_{i^{c}}^{i, l}$ and $\bigcup_{l \in A_{\varepsilon}} W_{i^{c}}^{i, l}$ are equivalent.

- The structure of $V$ from the equality $\sum_{v \in V} g(v, w)=2^{d}$. Let $V, W \subset S^{d}$ be disjoint sets which are equivalent polybox codes. Then for every $w \in W$ we have $w \sqsubseteq V, w \notin V$, 
and, by $(2.6), \sum_{v \in V} g(v, w)=2^{d}$, where $g(v, w) \in\left\{0,1,2, \ldots, 2^{d-1}\right\}$ for every $v \in V$. Assume that $w=b \ldots b$ and let $\left\{v^{1}, \ldots, v^{m}\right\} \subseteq V$ be such that $\breve{w} \cap \breve{v}^{i} \neq \emptyset$ for every $i \in[m]$ and $w \sqsubseteq\left\{v^{1}, \ldots, v^{m}\right\}$. The solutions of the system of the equations $\sum_{i=0}^{d-1} x_{i} 2^{i}=$ $2^{d}, \sum_{i=0}^{d-1} x_{i}=m$, where $x_{i}$ are non-negative integers for $i \in\{0,1, \ldots, d-1\}$, show the frequency of the letter $b$ in the words from the set $\left\{v^{1}, \ldots, v^{m}\right\}$. We explain this on the following example. Recall first that $g(v, w)=2^{i}$ if and only if $v_{j}=b$ for every $j \in I \subset[d],|I|=i$ and $v_{j} \notin\left\{b, b^{\prime}\right\}$ for $j \in[d] \backslash I$. In the example we assume that $d=3$, $w=b b b$ and $m=5$. The above system has two solutions: $x_{0}=2, x_{1}=3, x_{2}=0$ and $x_{0}=4, x_{1}=0, x_{2}=1$. It follows from the first solution that in the set $\left\{v^{1}, \ldots, v^{5}\right\}$ there are exactly three words such that each of them contains exactly one letter $b$ and two words which have no letter $b$ or, by the second solution, in the set $\left\{v^{1}, \ldots, v^{5}\right\}$ there is exactly one word with two letters $b$ and the rest four words have no letter $b$. This observation is quite useful in the computations as it allows us to restrict the number of words which have to be considered during the computations (see Section 5).

\subsection{Graph of siblings on a polybox code}

In the previous section we described slices of a polybox $\bigcup E(V)$ by the sets $\pi_{x}^{i}$. Observe that if the set of boxes $\left\{v_{i^{c}}: v \in V\right.$ and $\left.\pi_{x}^{i} \cap \breve{v} \neq \emptyset\right\}$ contains a twin pair, say $v_{i^{c}}, u_{i^{c}}$, and $V$ does not contain a twin pair, then $v_{i} \notin\left\{u_{i}, u_{i}^{\prime}\right\}$ (see Figure 10, the picture on the left). As we will see the knowledge of the number of such pairs $v, u$ in $V$ can help in the estimation of the number of words in the polybox code $V$.

In [10] we defined a graph on a polybox code $V$. We now recall the definition of it.

Two words $v, u \in S^{d}$ such that $v_{i} \notin\left\{u_{i}, u_{i}^{\prime}\right\}$ for some $i \in[d]$, and $v_{i^{c}}, u_{i^{c}}$ is a twin pair are called $i$-siblings (in Figure 10 the boxes on the left are a realization of $i$-siblings for $i=3)$.

Let $V \subseteq S^{d}$ be a polybox code. A graph of siblings on $V$ is a graph $G=(V, \mathscr{E})$ in which two vertices $v, u \in V$ are adjacent if they are $i$-siblings for some $i \in[d]$. We colour each edge in $\mathscr{E}$ with the colours from the set $[d]$ : An edge $(v, u) \in \mathscr{E}$ has a colour $i \in[d]$ if $v, u$ are $i$-siblings. The graph $G$ is simple and, if $V$ does not contain a twin pair, $d(v) \leqslant d$ for every $v \in V$, where $d(v)$ denotes the number of neighbors of $v$. (To show that $d(v) \leqslant d$, suppose on the contrary that it is not true. Then there are two vertices $u$ and $w$ which are adjacent to $v$ such that $v_{j}^{\prime}=u_{j}$ and $v_{j}^{\prime}=w_{j}$ for some $j \in[d]$. Since $v, u$ are $k$-siblings, and $v, w$ are $n$-siblings for some $k, n \in[d] \backslash\{j\}$, we have $v_{k} \notin\left\{u_{k}, u_{k}^{\prime}\right\}, v_{n} \notin\left\{w_{n}, w_{n}^{\prime}\right\}$ and $v_{\{k, j\}^{c}}=u_{\{k, j\}^{c}}, v_{\{n, j\}^{c}}=w_{\{n, j\}^{c}}$. The vertices $u$ and $w$ are dichotomous, and therefore it must be $u_{k}^{\prime}=w_{n}$ and $k=n$. Thus, $u, w$ are a twin pair, a contradiction.)

Observe that if $v, u$ are $i$-siblings in a polybox code $V$ such that $v_{i}=l$ and $u_{i}=s$, $l \notin\left\{s, s^{\prime}\right\}$, then for every $x \in E l \cap E s$ the set $\left\{v_{i^{c}}: v \in V\right.$ and $\left.\pi_{x}^{i} \cap \breve{v} \neq \emptyset\right\}$ contains the twin pair $v_{i^{c}}, u_{i^{c}}$ (see Figure 10).

In $[10]$ we proved the following two lemmas.

Lemma 6. Let $G=(V, \mathscr{E})$ be a graph of siblings on a polybox code $V \subset S^{d}$, u and $v$ be adjacent vertices, and let $d(u)=n$ and $d(v)=m$. If $n+m=2 d$, then there are $i \in[d]$ 
and $l \in S$ such that

$$
\left|V^{i, l} \cup V^{i, l^{\prime}}\right| \geqslant 2 d-2
$$

and if $n+m \leqslant 2 d-1$, then

$$
\left|V^{i, l} \cup V^{i, l^{\prime}}\right| \geqslant n+m-1
$$

for some $i \in[d]$ and some $l \in S$.

By $d(G)$ we denote the average degree of a graph $G$.

Lemma 7. Let $G=(V, \mathscr{E})$ be a simple graph, and let

$$
m=\max \{d(v)+d(u): v, u \in V \text { and } v, u \text { are adjacent }\} .
$$

Then $d(G) \leqslant m / 2$.

Lemma 8. Let $S=\left\{a, a^{\prime}, b, b^{\prime}\right\}$, and let $G=(V, \mathscr{E})$ be a graph of siblings on a polybox code $V \subset S^{d}$. In every cycle in $G$ the number of edges with a colour $i \in[d]$ is even. In particular, there are no odd cycles in $G$.

Proof. Let $v^{1},\left(v^{1}, v^{2}\right), v^{2}, \ldots, v^{n},\left(v^{n}, v^{1}\right), v^{1}$ be a cycle in $V, i \in[d]$, and let

$$
\left(v^{k_{1}}, v^{k_{1}+1}\right), \ldots,\left(v^{k_{j}}, v^{k_{j}+1}\right)
$$

be all edges in this cycle with the colour $i$, where $1 \leqslant k_{1}<\cdots<k_{j} \leqslant n$ and $k_{j}+1$ is taken modulo $n+1$. Assume that $v_{i}^{1}=a$. It follows from the definition of $i$-siblings that alternately $v_{i}^{m} \in\left\{a, a^{\prime}\right\}$ for every $m \in\left\{1, \ldots, k_{1}\right\}, v_{i}^{m} \in\left\{b, b^{\prime}\right\}$ for every $m \in$ $\left\{k_{1}+1, \ldots, k_{2}\right\}, v_{i}^{m} \in\left\{a, a^{\prime}\right\}$ for every $m \in\left\{k_{2}+1, \ldots, k_{3}\right\}, \ldots, v_{i}^{m} \in\left\{b, b^{\prime}\right\}$ for every $m \in\left\{k_{j-1}+1, \ldots, k_{j}\right\}$. Thus, $j$ is even.

\section{Small polybox codes}

In this section we collect basic results about polybox codes with a small number of words.

The proofs of the following two lemmas can be found in [10, Corollary 3.3 and 3.4].

Lemma 9. Let $V \subset S^{d}$ be a polybox code without twin pairs, and let $w \in S^{d}$ be a word such that $w \sqsubseteq V$ and $w \notin V$. Then the code $V$ contains at least five words. If the code $V$ has exactly five words, then there are a set $A=\left\{i_{1}<i_{2}<i_{3}\right\} \subseteq[d]$ and letters $l_{1}, l_{2}, l_{3} \in S$ such that

$$
V_{A}=\left\{l_{1} l_{2} l_{3}, l_{1}^{\prime} l_{2}^{\prime} l_{3}^{\prime}, w_{i_{1}} l_{2} l_{3}^{\prime}, l_{1}^{\prime} w_{i_{2}} l_{3}, l_{1} l_{2}^{\prime} w_{i_{3}}\right\} \quad \text { and } \quad V_{A^{c}}=\left\{w_{A^{c}}\right\}
$$

where $l_{k} \notin\left\{w_{i_{k}}, w_{i_{k}}^{\prime}\right\}$ for $k=1,2,3$. Moreover, the code $V$ is rigid, and for $d=3$ the code $V$ is the unique code without twin pair which can cover a word $w \in S^{3} \backslash V$. 
Lemma 10. Let $V \subset S^{d}$ be a polybox code without twin pairs, and let $w \in S^{d}$ be a word such that $w \sqsubseteq V$ and $\breve{w} \cap \breve{v} \neq \emptyset$ for every $v \in V$. If the code $V$ has exactly six words, then there are a set $A=\left\{i_{1}<i_{2}<i_{3}<i_{4}\right\} \subseteq[d]$ and letters $l_{1}, l_{2}, l_{3}, l_{4} \in S$ such that

$$
V_{A}=\left\{l_{1} l_{2} l_{3} l_{4}, l_{1}^{\prime} l_{2}^{\prime} l_{3}^{\prime} l_{4}, w_{i_{1}} l_{2} l_{3}^{\prime} l_{4}, l_{1}^{\prime} w_{i_{2}} l_{3} l_{4}, l_{1} l_{2}^{\prime} w_{i_{3}} l_{4}, w_{i_{1}} w_{i_{2}} w_{i_{3}} l_{4}^{\prime}\right\} \quad \text { and } \quad V_{A^{c}}=\left\{w_{A^{c}}\right\}
$$

where $l_{k} \notin\left\{w_{i_{k}}, w_{i_{k}}^{\prime}\right\}$ for $k=1,2,3,4$.

Lemma 11. Let $V \subset S^{d}$ be a polybox code without twin pairs. If words $w, u \in S^{d}$ are dichotomous, do not form a twin pair and $w, u \sqsubseteq V$ but $w, u \notin V$, then $|V| \geqslant 7$.

Proof. Let $W=\{v \in V: \breve{v} \cap \breve{w} \neq \emptyset\}$ and $U=\{v \in V: \breve{v} \cap \breve{u} \neq \emptyset\}$. By Lemma 9, $|W| \geqslant 5$ and $|U| \geqslant 5$. We will consider two cases: The first is $|W|=5$ and $|U|=5$, and the second is $|W|=6$.

Let $|W|=5$ and $|U|=5$. By Lemma 9 , there is a set $A=\left\{i_{1}<i_{2}<i_{3}\right\} \subset[d]$ and letters $l_{1}, l_{2}, l_{3} \in S, l_{k} \notin\left\{w_{i_{k}}, w_{i_{k}}^{\prime}\right\}$ for $k=1,2,3$, such that

$$
W_{A}=\left\{l_{1} l_{2} l_{3}, l_{1}^{\prime} l_{2}^{\prime} l_{3}^{\prime}, w_{i_{1}} l_{2} l_{3}^{\prime}, l_{1}^{\prime} w_{i_{2}} l_{3}, l_{1} l_{2}^{\prime} w_{i_{3}}\right\} \quad \text { and } \quad W_{A^{c}}=\left\{w_{A^{c}}\right\} .
$$

Clearly, if $|W \cap U| \leqslant 3$, then $|V| \geqslant 7$. Therefore, we may assume that $|W \cap U|=4$ or $|W \cap U|=5$.

Let $|W \cap U|=4$. Since $w$ and $u$ are dichotomous, there is $i \in[d]$ such that $w_{i}^{\prime}=u_{i}$. If $i \in A^{c}$, then $W \cap U=\emptyset$ because $w_{A^{c}}=v_{A^{c}}$ for every $v \in W$. Thus, $i \in A$. Assume without loss of generality that $i=i_{1}$. Since the form of $U$ is such as described in Lemma $9,|W \cap U|=4$ and $w_{i}^{\prime}=u_{i}$, it follows that

$$
U_{A}=\left\{l_{1} l_{2} l_{3}, l_{1}^{\prime} l_{2}^{\prime} l_{3}^{\prime}, w_{i_{1}}^{\prime} l_{2} l_{3}^{\prime}, l_{1}^{\prime} w_{i_{2}} l_{3}, l_{1} l_{2}^{\prime} w_{i_{3}}\right\} \quad \text { and } \quad U_{A^{c}}=\left\{w_{A^{c}}\right\} .
$$

Hence, $w_{i_{1}}^{\prime}=u_{i_{1}}, w_{i_{2}}=u_{i_{2}}, w_{i_{3}}=u_{i_{3}}$ and $w_{A^{c}}=u_{A^{c}}$. Then $w$ and $u$ are a twin pair, a contradiction.

If $|W \cap U|=5$, that is, $W=U$, then $u=w$, which contradicts the assumption.

To consider the case $|W|=6$, let us assume on the contrary that $|V|=6$. Then $V=W$. By Lemma 10,

$$
W_{A}=\left\{l_{1} l_{2} l_{3} l_{4}, l_{1}^{\prime} l_{2}^{\prime} l_{3}^{\prime} l_{4}, w_{i_{1}} l_{2} l_{3}^{\prime} l_{4}, l_{1}^{\prime} w_{i_{2}} l_{3} l_{4}, l_{1} l_{2}^{\prime} w_{i_{3}} l_{4}, w_{i_{1}} w_{i_{2}} w_{i_{3}} l_{4}^{\prime}\right\} \quad \text { and } \quad W_{A^{c}}=\left\{w_{A^{c}}\right\},
$$

where $A=\left\{i_{1}<i_{2}<i_{3}<i_{4}\right\} \subseteq[d]$ and $l_{k} \notin\left\{w_{i_{k}} . w_{i_{k}}^{\prime}\right\}$ for $k=1,2,3,4$.

Let $p \in W$ be such that $p_{A}=w_{i_{1}} w_{i_{2}} w_{i_{3}} l_{4}^{\prime}$. Since, by Lemma $9, p_{i_{4}^{c}} \sqsubseteq(W \backslash\{p\})_{i_{4}^{c}}$, we have $u_{i_{4}^{c}} \sqsubseteq(W \backslash\{p\})_{i_{4}^{c}}$. Indeed, if $u_{i_{4}} \notin\left\{l_{4}, l_{4}^{\prime}\right\}$, then $u_{i_{4}^{c}}=p_{i_{4}^{c}}$, for otherwise $u$ is not covered by $W$, which contradicts the assumption; if $u_{i_{4}}=l_{4}^{\prime}$, then $u=p$ which is impossible; if $u_{i_{4}}=l_{4}$, then $u \sqsubseteq W \backslash\{p\}$. Note that the form of the code $(W \backslash\{p\})_{i_{4}^{c}}$ is as in Lemma 9. In particular, $w_{i_{4}^{c}}$ is one and only word which is covered by the set $(W \backslash\{p\})_{i_{4}^{c}}$ and does not belong to it. Hence, $u_{i_{4}^{c}}=w_{i_{4}^{c}}$. Consequently, $w$ and $u$ are a twin pair, a contradiction. Thus, $|V| \geqslant 7$. 
Lemma 12. Let $V \subset S^{d}$ be a polybox code, and let $w \in S^{d}$ be a word such that $w \sqsubseteq V$. Assume that there are $i \in[d]$ and $l \in S, l \notin\left\{w_{i}, w_{i}^{\prime}\right\}$, such that the sets $Q=\left\{v \in V^{i, l}: \breve{w} \cap\right.$ $\breve{v} \neq \emptyset\}, P=\left\{v \in V^{i, l^{\prime}}: \breve{w} \cap \breve{v} \neq \emptyset\right\}$ are non-empty. Then the set $\breve{w} \cap \bigcup E\left(V^{i, l} \cup V^{i, l^{\prime}}\right)$ is an $i$-cylinder in the $d$-box $\breve{w}$ and consequently

$$
\bigcup\left\{(\breve{w} \cap \breve{v})_{i^{c}}: v \in Q\right\}=\bigcup\left\{(\breve{w} \cap \breve{v})_{i^{c}}: v \in P\right\} .
$$

Moreover, if $|P|=1$ and $1 \leqslant|Q| \leqslant 4$, then there is a twin pair in $V$.

Proof. The set $\breve{w} \cap \bigcup E\left(V^{i, l} \cup V^{i, l^{\prime}}\right)$ is an $i$-cylinder in the $d$-box $\breve{w}$ because the set of boxes $\mathscr{F}=\{\breve{w} \cap \breve{v}: v \in V\}$ is a suit for $\breve{w}$ (compare Example 4). Therefore, $\bigcup\left\{(\breve{w} \cap \breve{v})_{i^{c}}: v \in\right.$ $\left.V^{i, l}\right\}=\bigcup\left\{(\breve{w} \cap \breve{v})_{i^{c}}: v \in V^{i, l^{\prime}}\right\}$.

The proof the second part of the lemma can be found in [10, Lemma 4.1].

Lemma 13. Under the assumptions of Lemma 12, if the code $V$ does not contain a twin pair and $|P|=1$, then $|Q| \geqslant 5$ and $P_{i^{c}} \sqsubseteq Q_{i^{c}}$.

Proof. Let $P=\{u\}$. By Lemma 12, $|Q| \geqslant 5$ and the set $\breve{w} \cap \breve{u} \cup \bigcup\{\breve{w} \cap \breve{v}: v \in Q\}$ is an $i$-cylinder in the box $\breve{w}$. Then $(\breve{w} \cap \breve{u})_{i^{c}}=\bigcup\left\{(\breve{w} \cap \breve{v})_{i^{c}}: v \in Q\right\}$. Thus, the family $\mathscr{G}=\left\{(\breve{w} \cap \breve{v})_{i^{c}}: v \in Q\right\}$ is a suit for the $(d-1)$-box $(\breve{w} \cap \breve{u})_{i^{c}}$, and then $E w_{j} \cap E v_{j} \subseteq$ $E w_{j} \cap E u_{j}$ for every $j \in[d] \backslash\{i\}$ and $v \in Q$. Therefore, by (2.3), if $w_{j} \neq u_{j}$, then $v_{j}=u_{j}$ for every $v \in Q$. Indeed, if on the contrary $w_{j} \neq u_{j}$ and $v_{j} \neq u_{j}$ for some $j \in[d] \backslash\{i\}$ and some $v \in V$, then, by (2.3), there is a point $x \in E w_{j} \cap E v_{j}$ such that $x \notin E w_{j} \cap E u_{j}$, and then $E w_{j} \cap E v_{j} \nsubseteq E w_{j} \cap E u_{j}$, a contradiction. Let $D=\left\{j \in[d] \backslash\{i\}: w_{j}=u_{j}\right\}$ and $D^{c}=[d] \backslash(D \cup\{i\})$. Since $u_{D^{c}}=v_{D^{c}}$ for every $v \in Q$ and $\breve{u}_{D} \subseteq \bigcup_{v \in Q} \breve{v}_{D}$, and consequently, by $(2.6), u_{D} \sqsubseteq Q_{D}$ (because $w_{j}=u_{j}$ for $j \in D$ and $\mathscr{G}$ is a suit for $(\breve{w} \cap \breve{u})_{i^{c}}$ ), it follows that $u_{i^{c}} \sqsubseteq Q_{i^{c}}$.

The last lemma of this section describes a general form of the configuration of four words which appeared in Example 4. The proof of this lemma can be found in [10, Lemma $3.1]$.

Lemma 14. Under the assumptions of Lemma 12, if the code $V$ does not contain a twin pair and $|P|=|Q|=2$, then there are a set $A=\left\{i_{1}<i_{2}\right\} \subseteq[d] \backslash\{i\}$ and letters $l_{1}, l_{2} \in S$ such that

$$
P_{A}=\left\{w_{i_{1}} l_{2}, l_{1}^{\prime} l_{2}^{\prime}\right\} \quad \text { and } \quad Q_{A}=\left\{l_{1} l_{2}, l_{1}^{\prime} w_{i_{2}}\right\}
$$

where $l_{k} \notin\left\{w_{i_{k}}, w_{i_{k}}^{\prime}\right\}$ for $k=1,2$. Moreover, $P_{A^{c}}=Q_{A^{c}}=\left\{p_{A^{c}}\right\}$ for some $p \in S^{d}$, where $A^{c}=[d] \backslash\left\{i, i_{1}, i_{2}\right\}$.

\section{The structure of equivalent polyboxes codes with 12 words: Necessary conditions}

To show that Keller's conjecture is true in dimension seven for a cube tiling $[0,1)^{7}+T$ for which $r^{+}(T) \geqslant 6$, it was sufficient to prove the following theorem ([10]): 
Theorem 15. If $V, W \subset S^{d}$ are disjoint sets which are equivalent polybox codes without twin pairs, then $|V| \geqslant 12$.

To show that the conjecture is true in dimension seven for a cube tiling $[0,1)^{7}+T$ with $r^{+}(T)=5$, we will describe the structure of all twin pair free disjoint and equivalent polybox codes $V$ and $W$, with 12 words each, in dimensions four, five and six (compare Section 2.4).

In this section we determine necessary conditions which have to be fulfilled by such the codes $V$ and $W$.

It was shown in [10] that a polybox code without twin pairs having at most seven words is rigid. Now we need a slightly better rigidity result:

Lemma 16. Let $S=\left\{a, a^{\prime}, b, b^{\prime}\right\}$. If a polybox code $V \subset S^{d}$ does not contain a twin pair and $|V| \leqslant 9$, then it is rigid.

Proof. We proceed by induction on $d$. By Lemma 9 , for $d \leqslant 3$ every polybox code $V \subset S^{d}$ without twin pairs is rigid. Thus, the lemma is true for $d \leqslant 3$. Let $d \geqslant 4$. Suppose on the contrary that there is a polybox code $V \subset S^{d}$ having at most nine words which is not rigid. Thus, there is a polybox code $W \subset S^{d}$ which is equivalent to $V$ and $V \cap W=\emptyset$. (It can happen that $W$ contains a twin pair.) We will show first that $V^{i, l} \neq \emptyset$ for every $i \in[d]$ and $l \in S$.

Suppose that this is not true. We may assume that $V^{i, a} \neq \emptyset$ and $V^{i, b}=\emptyset$ for some $i \in[d]$. By Lemma 5, the polybox codes $V_{i^{c}}^{i, a}$ and $W_{i^{c}}^{i, a} \cup W_{i^{c}}^{i, b}$ are equivalent, and by the inductive hypothesis the code $V_{i^{c}}^{i, a} \subset S^{d-1}$ is rigid. Thus, $V_{i^{c}}^{i, a}=W_{i^{c}}^{i, a} \cup W_{i^{c}}^{i, b}$. Since $V$ and $W$ are disjoint, we have $V_{i^{c}}^{i, a}=W_{i^{c}}^{i, b}$. Then, by (Co) in Section 2.3, $V_{i^{c}}^{i, a} \sqsubseteq V_{i^{c}}^{i, a^{\prime}}$, and thus $V^{i, a^{\prime}} \neq \emptyset$. In the same way we show that $V_{i^{c}}^{i, a^{\prime}} \sqsubseteq V_{i^{c}}^{i, a}$. Consequently the codes $V_{i^{c}}^{i, a^{\prime}}, V_{i^{c}}^{i, a}$ are equivalent. Since $V_{i^{c}}^{i, a}$ is rigid, these codes are equal. Then the set $V^{i, a} \cup V^{i, a^{\prime}}$ contains a twin pair, which is impossible.

Thus, $V^{i, l} \neq \emptyset$ for every $i \in[d]$ and $l \in S$.

We now show that for every $l, s \in S, l \notin\left\{s, s^{\prime}\right\}$, the code $V^{i, l} \cup V^{i, s}$ contains $i$-siblings.

To do this, let us suppose on the contrary that there are $i \in[d]$ and two letters in $S$, say $a$ and $b$, such that there are no $i$-siblings in $V^{i, a} \cup V^{i, b}$, that is, the polybox code $V_{i^{c}}^{i, a} \cup V_{i^{c}}^{i, b}$ does not contain a twin pair. This means, by the inductive hypothesis, that the polybox code $V_{i^{c}}^{i, a} \cup V_{i^{c}}^{i, b}$ is rigid. Then, by Lemma $5, V_{i^{c}}^{i, a} \cup V_{i^{c}}^{i, b}=W_{i^{c}}^{i, a} \cup W_{i^{c}}^{i, b}$. Since $V \cap W=\emptyset$, it follows that $V_{i^{c}}^{i, a}=W_{i^{c}}^{i, b}$ and $V_{i^{c}}^{i, b}=W_{i^{c}}^{i, a}$. By $(\mathbf{C o}), V_{i^{c}}^{i, a} \sqsubseteq V_{i^{c}}^{i, a^{\prime}}$ and $V_{i^{c}}^{i, b} \sqsubseteq V_{i^{c}}^{i, b^{\prime}}$. Note that $V_{i^{c}}^{i, a} \cap V_{i^{c}}^{i, a^{\prime}}=\emptyset$ and $V_{i^{c}}^{i, b} \cap V_{i^{c}}^{i, b^{\prime}}=\emptyset$ because $V$ does not contain twin pairs. Therefore, by Lemma $9,|V| \geqslant\left|V^{i, a^{\prime}}\right|+\left|V^{i, b^{\prime}}\right| \geqslant 10$, a contradiction.

Thus, we assume that for every $l, s \in\left\{a, a^{\prime}, b, b^{\prime}\right\}, l \notin\left\{s, s^{\prime}\right\}$ there are $i$-siblings in $V^{i, l} \cup V^{i, s}$.

We now consider a graph $G=(V, \mathscr{E})$ of siblings on $V$ (compare Section 2.9). Recall that $N(v)$ denotes the set of all neighbors of the vertex $v \in V$.

It follows from the above assumption on $i$-siblings in $V$ that for every $\{l, s\} \in\{\{b, a\}$, $\left.\left\{b, a^{\prime}\right\},\left\{b^{\prime}, a\right\},\left\{b^{\prime}, a^{\prime}\right\}\right\}$ and every $i \in[d]$ there is an edge $(v, u) \in \mathscr{E}$ such that $\left\{v_{i}, u_{i}\right\}=$ 
$\{l, s\}$. Thus, for every $i \in[d]$ there are at least 4 edges with the colour $i$, and therefore $|\mathscr{E}| \geqslant 4 d$.

Let $u^{0}, v^{0} \in V$ be such that

$$
d\left(v^{0}\right)+d\left(u^{0}\right)=\max \{d(v)+d(u): v, u \in V \text { and } v, u \text { are adjacent }\} .
$$

We will consider three cases: $d\left(u^{0}\right)+d\left(v^{0}\right)=9, \quad d\left(u^{0}\right)+d\left(v^{0}\right)=8$ and finally $d\left(u^{0}\right)+$ $d\left(v^{0}\right) \leqslant 7$.

Let $d\left(u^{0}\right)+d\left(v^{0}\right)=9$. By Lemma 6 (2.8), there are $i \in[d]$ and $l \in S$ such that $\left|V^{i, l} \cup V^{i, l^{\prime}}\right| \geqslant 8$. Since $V^{i, s} \neq \emptyset$ and $V^{i, s^{\prime}} \neq \emptyset$, where $s \notin\left\{l, l^{\prime}\right\}$, it follows that $|V| \geqslant 10$, a contradiction.

Let $d\left(u^{0}\right)+d\left(v^{0}\right)=8$. We first assume that $d \geqslant 5$.

We may assume without loss of generality that $u^{0}=a a a \ldots a$ and $v^{0}=b a^{\prime} a \ldots a$. If there are $i \in[d]$ and $l \in S$ such that $\left|V^{i, l} \cup V^{i, l^{\prime}}\right| \geqslant 8$, then $|V| \geqslant 10$ because $\left|V^{i, s}\right| \geqslant$ 1 and $\left|V^{i, s^{\prime}}\right| \geqslant 1$, where $s \notin\left\{l, l^{\prime}\right\}$, which is impossible. Thus, we may assume that $\left|V^{i, l} \cup V^{i, l^{\prime}}\right| \leqslant 7$ for every $i \in[d]$ and $l \in S$. Note that there are $i, j \in\{2, \ldots, d\}, i \neq j$, such that $\left|V^{i, a} \cup V^{i, a^{\prime}}\right|=7,\left|V^{j, a} \cup V^{j, a^{\prime}}\right|=7$ and the sets $V^{i, a} \cup V^{i, a^{\prime}}$ and $V^{j, a} \cup V^{j, a^{\prime}}$ are subsets of $N\left(u^{0}\right) \cup N\left(v^{0}\right)$. (To see this, observe first that for every $v \in N\left(u^{0}\right) \cup N\left(v^{0}\right)$ the word $v$ has at most one letter from the set $\left\{b, b^{\prime}\right\}$ at the positions $2, \ldots, d$. Since $\left|V^{i, l} \cup V^{i, l^{\prime}}\right| \leqslant 7$ for every $i \in[d]$ and $l \in S$, it follows that for every $i \in[d]$ there is at least one $v \in N\left(u^{0}\right) \cup N\left(v^{0}\right)$ with $v_{i} \in\left\{b, b^{\prime}\right\}$. Therefore, there are at most two positions $n, k \in\{2, \ldots, d\}$ such that $v_{n} \in\left\{b, b^{\prime}\right\}$ for at least two words $v \in N\left(u^{0}\right) \cup N\left(v^{0}\right)$ and $v_{k} \in\left\{b, b^{\prime}\right\}$ for at least two words $v \in N\left(u^{0}\right) \cup N\left(v^{0}\right)$. Consequently, there are two positions $i, j \in\{2, \ldots, d\} \backslash\{n, k\}$ such that $u_{i} \in\left\{b, b^{\prime}\right\}$ for precisely one $u \in N\left(u^{0}\right) \cup N\left(v^{0}\right)$ and $w_{j} \in\left\{b, b^{\prime}\right\}$ for precisely one $w \in N\left(u^{0}\right) \cup N\left(v^{0}\right)$.)

Let $\{u\}=\left(N\left(u^{0}\right) \cup N\left(v^{0}\right)\right) \backslash\left(V^{i, a} \cup V^{i, a^{\prime}}\right)$ and $\{w\}=\left(N\left(u^{0}\right) \cup N\left(v^{0}\right)\right) \backslash\left(V^{j, a} \cup V^{j, a^{\prime}}\right)$. We have $u_{i} \in\left\{b, b^{\prime}\right\}, u_{j} \in\left\{a, a^{\prime}\right\}$ and $w_{j} \in\left\{b, b^{\prime}\right\}, w_{i} \in\left\{a, a^{\prime}\right\}$. Moreover, $\bar{v}_{i}, \bar{v}_{j} \in\left\{b, b^{\prime}\right\}$, where $\{\bar{v}\}=V \backslash\left(N\left(u^{0}\right) \cup N\left(v^{0}\right)\right)$. Assume without loss of generality that $u_{i}=b$ and $w_{i}=a$. Since $v_{i}, v_{j} \in\left\{a, a^{\prime}\right\}$ for every $v \in\left(N\left(u^{0}\right) \cup N\left(v^{0}\right)\right) \backslash\{u, w\}$, the only vertices from the set $N\left(u^{0}\right) \cup N\left(v^{0}\right)$ which can be adjacent to the vertex $\bar{v}$ are $u$ and $w$. This means that, there are no two words $p, q \in V$ which are $i$-siblings such that $p_{i}=b^{\prime}$ and $q_{i}=a^{\prime}$. A contradiction.

Let now $d=4$ and assume that $u^{0}=a a a a$ and $v^{0}=b a^{\prime} a a$. By just considered case, we assume that for every $i, j \in\{2,3,4\}, i \neq j$, we have $\left|V^{i, a} \cup V^{i, a^{\prime}}\right| \leqslant 6$ or $\left|V^{j, a} \cup V^{j, a^{\prime}}\right| \leqslant 6$. Thus, it suffices to consider three cases: $n_{2}=n_{3}=n_{4}=2 ; n_{2}=3, n_{3}=2, n_{4}=1$, and $n_{2}=n_{3}=2, n_{4}=1$, where

$$
n_{i}=\left|\left(V^{i, b} \cup V^{i, b^{\prime}}\right) \cap\left(N\left(u^{0}\right) \cup N\left(v^{0}\right)\right)\right|
$$

for $i \in\{2,3,4\}$.

Let $\left\{v^{1}, \ldots, v^{6}\right\}=\left(N\left(u^{0}\right) \cup N\left(v^{0}\right)\right) \backslash\left\{u^{0}, v^{0}\right\}$. Observe that in the first two cases, if two vertices $v^{n}, v^{m} \in\left\{v^{1}, \ldots, v^{6}\right\}$ are adjacent, then $v^{n}, v^{m} \in\left(V^{i, b} \cup V^{i, b^{\prime}}\right) \cap\left(N\left(u^{0}\right) \cup N\left(v^{0}\right)\right)$ for some $i \in\{2,3,4\}$. (Recall that, since $u^{0}=a a a a$ and $v^{0}=b a^{\prime} a a$, if $v_{i} \in\left\{b, b^{\prime}\right\}$ for some $v \in N\left(v^{0}\right) \cup N\left(u^{0}\right)$ and $i \in\{2,3,4\}$, then $v_{j} \in\left\{a, a^{\prime}\right\}$ for every $j \in\{2,3,4\} \backslash\{i\}$.) 
Therefore, in these two cases the maximal number of edges with endpoints in $N\left(u^{0}\right) \cup N\left(v^{0}\right)$ is achieved if the vertices $v^{1}, \ldots, v^{6}$ are arranged as presented in Figure $11 a$ and $b$ for the first and the second case, respectively (recall that, by Lemma 8, the graph $G$ does not contain triangles). Since $d(\bar{v}) \leqslant 4$, where $\{\bar{v}\}=V \backslash\left(N\left(u^{0}\right) \cup N\left(v^{0}\right)\right)$, we have $|\mathscr{E}|<16$, which contradicts the assumption on the number of edges in $G$.

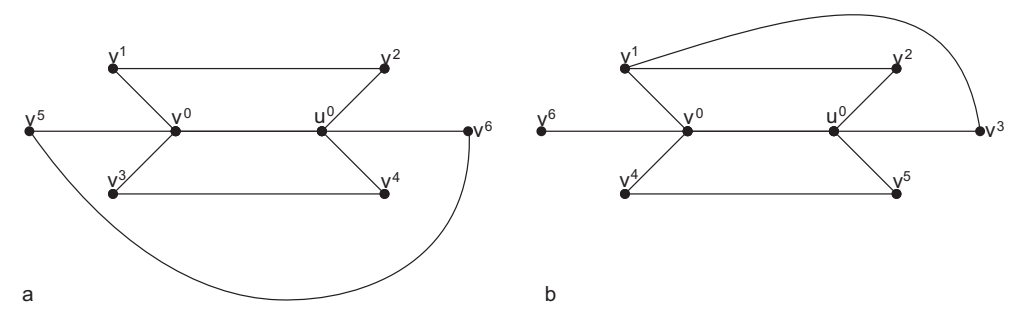

Figure 11: Subgraphs of $G$ with the maximal numbers of edges with endpoints in the set $N\left(u^{0}\right) \cup N\left(v^{0}\right)$ in the first two cases. In the first case $(a)$ we have $v_{2}^{1}, v_{2}^{2} \in\left\{b, b^{\prime}\right\}$, $v_{3}^{3}, v_{3}^{4} \in\left\{b, b^{\prime}\right\}$ and $v_{4}^{5}, v_{4}^{6} \in\left\{b, b^{\prime}\right\}$. In the second case $(b)$ we have $v_{2}^{1}, v_{2}^{2}, v_{2}^{3} \in\left\{b, b^{\prime}\right\}$, $v_{3}^{4}, v_{3}^{5} \in\left\{b, b^{\prime}\right\}$ and $v_{4}^{6} \in\left\{b, b^{\prime}\right\}$.

In the third case we assume that $v_{1}^{1} \in\left\{a, a^{\prime}, b, b^{\prime}\right\}$ and $v_{i}^{1} \in\left\{a, a^{\prime}\right\}$ for $i \in\{2,3,4\}$, $v_{2}^{2}, v_{2}^{3} \in\left\{b, b^{\prime}\right\}, v_{3}^{4}, v_{3}^{5} \in\left\{b, b^{\prime}\right\}$ and $v_{4}^{6} \in\left\{b, b^{\prime}\right\}$. Observe that now the vertices $v^{2}, \ldots, v^{6}$ can be joined with $v^{1}$, but similarly as above if $v^{n}, v^{m} \in\left\{v^{2}, \ldots, v^{6}\right\}$ are adjacent, then $v_{i}^{n}, v_{i}^{m} \in\left(V^{i, b} \cup V^{i, b^{\prime}}\right) \cap\left(N\left(u^{0}\right) \cup N\left(v^{0}\right)\right)$ for some $i \in\{2,3,4\}$. Since $d(\bar{v}) \leqslant 4$, the number of edges with endpoints in the set $N\left(u^{0}\right) \cup N\left(v^{0}\right)$ has to be at least 12 because $|\mathscr{E}| \geqslant 16$. This can be done only if $v^{1}, v^{3}, v^{5} \in N\left(u^{0}\right)$ and $v^{2}, v^{4}, v^{6} \in N\left(v^{0}\right)$ or $v^{1}, v^{2}, v^{4} \in N\left(v^{0}\right)$ and $v^{3}, v^{5}, v^{6} \in N\left(u^{0}\right)$. We will consider the first case (the second case is considered in the same way.) To obtain 12 edges with endpoints in the set $N\left(u^{0}\right) \cup N\left(v^{0}\right)$, which is the maximal number of edges with endpoints in this set, the vertices $v^{1}, \ldots, v^{6}$ have to be arranged as presented in Figure 12.

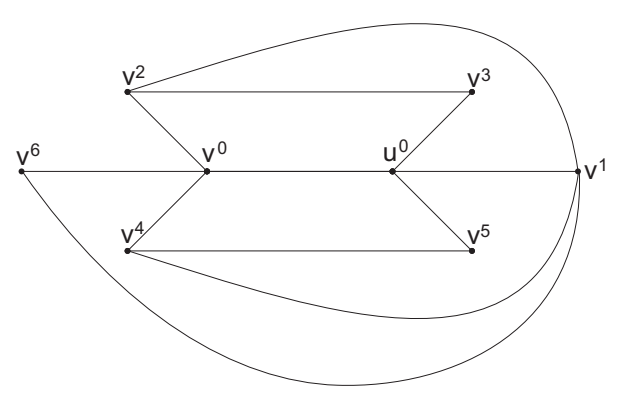

Figure 12: Subgraph of $G$ with the maximal number of edges with endpoints in the set $N\left(u^{0}\right) \cup N\left(v^{0}\right)$ in the third cases.

Since $d(v) \leqslant 4$ for every $v \in V$ and the graph $G$, by Lemma 8 , does not contain triangles, it must be $d(\bar{v})<4$ and then $|\mathscr{E}|<16$, a contradiction. This completes the proof of the lemma in the case $d\left(u^{0}\right)+d\left(v^{0}\right)=8$. 
Finally, let $d\left(v^{0}\right)+d\left(u^{0}\right) \leqslant 7$. It follows from Lemma 7 that

$$
d(G) \leqslant \frac{7}{2}
$$

Since $d(G)|V|=2|\mathscr{E}|$ and $2|\mathscr{E}| \geqslant 32$, we have $|V|>9$, a contradiction.

In the next two lemmas we give forbidden distributions of words in the codes $V$ and $W$ under consideration.

Lemma 17. Let $S=\left\{a, a^{\prime}, b, b^{\prime}\right\}$, and let $V, W \subset S^{d}$ be disjoint sets which are equivalent polybox codes without twin pairs. If $|V|=12$, then for every $i \in[d]$ and every $l, s \in S$, $l \notin\left\{s, s^{\prime}\right\}$, the distribution

$$
\left|V^{i, l}\right|=5,\left|V^{i, l^{\prime}}\right|=1,\left|V^{i, s}\right|=5,\left|V^{i, s^{\prime}}\right|=1
$$

is impossible.

Proof. Recall that $V_{i^{c}}^{i, l}=\left(V^{i, l}\right)_{i^{c}}$ for every $l \in S$ and $i \in[d]$.

Assume on the contrary that there is $i \in[d]$ such that $\left|V^{i, a}\right|=5,\left|V^{i, a^{\prime}}\right|=1$ and $\left|V^{i, b}\right|=5,\left|V^{i, b^{\prime}}\right|=1$. Let $\{v\}=V^{i, a^{\prime}}$ and $\{u\}=V^{i, b^{\prime}}$. Observe that there is $w \in W$ such that $\breve{w} \cap \bigcup E\left(V^{i, a}\right) \neq \emptyset$ and $\breve{w} \cap \bigcup E\left(V^{i, a^{\prime}}\right) \neq \emptyset$, for otherwise, by $(\mathbf{P})$ in Section 2.3, $V^{i, a} \sqsubseteq W^{i, a}$ and $V^{i, a^{\prime}} \sqsubseteq W^{i, a^{\prime}}$. Then, by Lemma 11 and $9,\left|W^{i, a}\right| \geqslant 7$ and $\left|W^{i, a^{\prime}}\right| \geqslant 5$, respectively. Hence, $W^{i, b} \cup W^{i, b^{\prime}}=\emptyset$ which is impossible because, by $(\mathbf{V})$ in Section 2.3, $\left|W^{i, b}\right| \geqslant 4$. Similarly, $\breve{w} \cap \bigcup E\left(V^{i, b}\right) \neq \emptyset$ and $\breve{w} \cap \bigcup E\left(V^{i, b^{\prime}}\right) \neq \emptyset$ for some $w \in W$.

Thus, by Lemma $13, v_{i^{c}} \sqsubseteq V_{i^{c}}^{i, a}$ and $u_{i^{c}} \sqsubseteq V_{i^{c}}^{i, b}$.

The proof splits into two parts. In the first part we assume that $u$ and $v$ are not $i$-siblings. In the second, the words $u, v$ are $i$-siblings.

Assume that $u, v$ are not $i$-siblings. Then the polybox code $\left\{u_{i^{c}}, v_{i^{c}}\right\}$ is rigid (see Figure 10, on the right). By Lemma 5, the polybox codes $V_{i c}^{i, a^{\prime}} \cup V_{i c}^{i, b^{\prime}}$ and $W_{i^{c}}^{i, a^{\prime}} \cup W_{i c}^{i, b^{\prime}}$ are equivalent. Thus, $\left\{v_{i^{c}}, u_{i^{c}}\right\}=W_{i^{c}}^{i, a^{\prime}} \cup W_{i^{c}}^{i, b^{\prime}}$. Since $V \cap W=\emptyset$, it follows that

$$
v_{i^{c}}=w_{i^{c}} \quad \text { and } \quad u_{i^{c}}=q_{i^{c}},
$$

where $\{w\}=W^{i, b^{\prime}}$ and $\{q\}=W^{i, a^{\prime}}$. By (Co) in Section 2.3, we have $v_{i^{c}} \sqsubseteq W_{i^{c}}^{i, b}$, $u_{i^{c}} \sqsubseteq W_{i^{c}}^{i, a}$. Thus, by Lemma $9,\left|W^{i, b}\right| \geqslant 5$ and $\left|W^{i, a}\right| \geqslant 5$. Hence, the code $W$ has the same distribution of words as the code $V:\left|W^{i, a}\right|=5,\left|W^{i, a^{\prime}}\right|=1$ and $\left|W^{i, b}\right|=5$, $\left|W^{i, b^{\prime}}\right|=1$.

Since $v_{i^{c}} \sqsubseteq W_{i^{c}}^{i, b}$ and $v_{i^{c}} \sqsubseteq V_{i^{c}}^{i, a}$, the forms of $W_{i^{c}}^{i, b}$ and $V_{i^{c}}^{i, a}$ are as described in Lemma 9. Similarly, $u_{i^{c}} \sqsubseteq W_{i^{c}}^{i, a}$ and $u_{i^{c}} \sqsubseteq V_{i^{c}}^{i, b}$, and therefore the forms of $W_{i^{c}}^{i, a}$ and $V_{i^{c}}^{i, b}$ are also as in Lemma 9.

Suppose that there is a point $y \in(E S)^{d}$ such that $y_{i^{c}} \notin \breve{v}_{i^{c}}$ and $y_{i^{c}} \in \bigcup E\left(W_{i^{c}}^{i, b}\right) \cap$ $\bigcup E\left(V_{i^{c}}^{i, a}\right)$. Then, by (2.3), we can choose the point $y$ such that $y_{i} \in E b \backslash E a$. Then, $y \in$ $\bigcup E\left(W^{i, b}\right)$ and $y \notin \bigcup E\left(V^{i, a}\right)$. Thus, $y \in \bigcup E(W)$ and $y \notin \bigcup E(V)$ because $\{v\}=V^{i, a^{\prime}}$. A contradiction. 
Therefore, the sets $\bigcup E\left(W_{i^{c}}^{i, b}\right)$ and $\bigcup E\left(V_{i^{c}}^{i, a}\right)$ cannot have a common point outside the box $\breve{v}_{i^{c}}$, and, for the same reason, the sets $\bigcup E\left(W_{i^{c}}^{i, a}\right)$ and $\bigcup E\left(V_{i^{c}}^{i, b}\right)$ cannot have a common point outside the box $\breve{u}_{i^{c}}$. Then, by $(\mathbf{P})$ and $(\mathbf{V})$, it must be

$$
\bigcup E\left(W_{i^{c}}^{i, b}\right) \backslash \breve{v}_{i^{c}}=\bigcup E\left(V_{i^{c}}^{i, b}\right) \backslash \breve{u}_{i^{c}} \text { and } \bigcup E\left(W_{i^{c}}^{i, a}\right) \backslash \breve{u}_{i^{c}}=\bigcup E\left(V_{i^{c}}^{i, a}\right) \backslash \breve{v}_{i^{c}} .
$$

We now examine when the sets $\bigcup E\left(W_{i^{c}}^{i, b}\right)$ and $\bigcup E\left(V_{i^{c}}^{i, a}\right)$ have a common point outside the box $\breve{v}_{i^{c}}$. Assume for simplicity that $v_{i^{c}}=b \ldots b$. Since $v_{i^{c}} \sqsubseteq W_{i^{c}}^{i, b}$, by Lemma 9 , there is a set $A=\left\{i_{1}<i_{2}<i_{3}\right\} \subseteq[d] \backslash\{i\}$ such that $\left(W_{i^{c}}^{i, b}\right)_{A}=\left\{l_{1} l_{2} l_{3}, l_{1}^{\prime} l_{2}^{\prime} l_{3}^{\prime}, b l_{2} l_{3}^{\prime}, l_{1}^{\prime} b l_{3}, l_{1} l_{2}^{\prime} b\right\}$, where $l_{k} \in\left\{a, a^{\prime}\right\}$ for $k=1,2,3$, and $\left(W_{i^{c}}^{i, b}\right)_{A^{c}}=\{b \ldots b\}$ (here $A^{c}=[d] \backslash(A \cup\{i\})$ ). Similarly, since $v_{i^{c}} \sqsubseteq V_{i^{c}}^{i, a}$, there is a set $B=\left\{j_{1}<j_{2}<j_{3}\right\} \subseteq[d] \backslash\{i\}$ such that $\left(V_{i^{c}}^{i, a}\right)_{B}=\left\{s_{1} s_{2} s_{3}, s_{s}^{\prime} s_{2}^{\prime} s_{3}^{\prime}, b s_{2} s_{3}^{\prime}, s_{1}^{\prime} b s_{3}, s_{1} s_{2}^{\prime} b\right\}$, where $s_{k} \in\left\{a, a^{\prime}\right\}$ for $k=1,2,3$, and $\left(V_{i^{c}}^{i, a}\right)_{B^{c}}=\{b \ldots b\}$ (here $B^{c}=[d] \backslash(B \cup\{i\})$ ). We will show that if $A \cap B \neq \emptyset$, then there is a point $y \in(E S)^{d}$ such that $y_{i^{c}} \in \bigcup E\left(W_{i^{c}}^{i, b}\right) \cap \bigcup E\left(V_{i^{c}}^{i, a}\right)$ and $y_{i^{c}} \notin \breve{v}_{i^{c}}$. We consider the case when $A=B$.

We may assume that $l_{1} l_{2} l_{3}=a a a$. If $s_{1} s_{2} s_{3}=a a a$, then taking $y_{i_{k}} \in E a \backslash E b$ for $k=1,2,3$ and $y_{j} \in E b$ for every $j \in A^{c}$ we obtain the point $y_{i^{c}}$ with the above mentioned property. Indeed, $y_{i^{c}} \in \breve{p}_{i^{c}} \cap \breve{t}_{i^{c}}$, where the words $p \in W^{i, b}, t \in V^{i, a}$ are such that $p_{A}=t_{A}=a a a$. Moreover, $y_{i^{c}} \notin \breve{v}_{i^{c}}$ because $y_{i_{k}} \in E a \backslash E b$ for $k=1,2,3$.

Let $s_{1} s_{2} s_{3} \notin\left\{a a a, a^{\prime} a^{\prime} a^{\prime}\right\}$. Since $\left\{s_{1} s_{2} s_{3}, s_{1}^{\prime} s_{2}^{\prime} s_{3}^{\prime}\right\} \in\left\{\left\{a^{\prime} a a, a a^{\prime} a^{\prime}\right\},\left\{a a^{\prime} a, a^{\prime} a a^{\prime}\right\},\left\{a a a^{\prime}\right.\right.$, $\left.\left.a^{\prime} a^{\prime} a\right\}\right\}$, we may assume that $s_{1} s_{2} s_{3}=a^{\prime} a a$. Then the set $\left(V_{i^{c}}^{i, a}\right)_{A}$ (we have $A=B$ ) contains one of the words $a a b$ or $a^{\prime} a^{\prime} b$; if it contains the word $a a b$, we take $y_{i_{k}} \in E a \backslash E b$ for $k=1,2, y_{i_{3}} \in E a \cap E b$ and $y_{j} \in E b$ for every $j \in A^{c}$; if it contains the word $a^{\prime} a^{\prime} b$, we take $y_{i_{k}} \in E a^{\prime} \backslash E b$ for $k=1,2, y_{i_{3}} \in E a^{\prime} \cap E b$ and $y_{j} \in E b$ for every $j \in A^{c}$. Since aaa, $a^{\prime} a^{\prime} a^{\prime} \in\left(W_{i^{c}}^{i, b}\right)_{A}$, in both cases $y_{i^{c}} \in \bigcup E\left(W_{i^{c}}^{i, b}\right) \cap \bigcup E\left(V_{i^{c}}^{i, a}\right)$. Clearly, $y_{i^{c}} \notin \breve{v}_{i^{c}}$ because $y_{i_{k}} \notin E b$ for $k=1,2$.

The rest two cases $|A \cap B|=2$ and $|A \cap B|=1$ are considered in the very similar way.

Thus, we assume that $A \cap B=\emptyset$. To finish this part of the proof we show that the equalities (4.1) cannot hold simultaneously.

We still may assume that $v_{i^{c}}=b \ldots b$. Since $u_{i^{c}}$ and $v_{i^{c}}$ do not form a twin pair, there is $j \in[d] \backslash\{i\}$ such that $u_{j} \notin\left\{b, b^{\prime}\right\}$. If $j \in A^{c}$, then $\bigcup E\left(W_{i^{c}}^{i, b}\right) \backslash \breve{v}_{i^{c}} \neq \bigcup E\left(V_{i^{c}}^{i, b}\right) \backslash \breve{u}_{i^{c}}$ because all words in $W_{i^{c}}^{i, b}$ have the letter $b$ at the position $j$, while, by Lemma 9 , there is a word $p_{i^{c}} \in V_{i^{c}}^{i, b}$ with $p_{j}=u_{j}$ and $\breve{p}_{i^{c}} \backslash \breve{u}_{i^{c}} \neq \emptyset$. To see that $\bigcup E\left(W_{i^{c}}^{i, b}\right) \backslash \breve{v}_{i^{c}} \neq \bigcup E\left(V_{i^{c}}^{i, b}\right) \backslash \breve{u}_{i^{c}}$ let us choose a point $z \in \breve{p}_{i^{c}} \backslash \breve{u}_{i^{c}}$ such that $z_{j} \in E p_{j} \backslash E b$. Then $z \in \bigcup E\left(V_{i^{c}}^{i, b}\right) \backslash \breve{u}_{i^{c}}$ and $z \notin \bigcup E\left(W_{i^{c}}^{i, b}\right) \backslash \breve{v}_{i^{c}}$.

Similarly, $\bigcup E\left(W_{i^{c}}^{i, a}\right) \backslash \breve{u}_{i^{c}} \neq \bigcup E\left(V_{i^{c}}^{i, a}\right) \backslash \breve{v}_{i^{c}}$ when $j \in B^{c}$. Since $A \cap B=\emptyset$, we have $[d] \backslash\{i\}=A^{c} \cup B^{c}$, and thus one of the equalities (4.1) does not hold. A contradiction. This finishes the proof of the lemma in the case when $u$ and $v$ are not $i$-siblings

Let now $u$ and $v$ be $i$-siblings. Then $v_{i^{c}}$ and $u_{i^{c}}$ are a twin pair. We can assume without loss of generality that $v_{i^{c}}=b \ldots b$. It was shown at the beginning of the proof, that $v_{i^{c}} \sqsubseteq V_{i^{c}}^{i, a}$. Thus, by Lemma $9,\left(V_{i^{c}}^{i, a}\right)_{A}=\left\{l_{1} l_{2} l_{3}, l_{1}^{\prime} l_{2}^{\prime} l_{3}^{\prime}, b l_{2} l_{3}^{\prime}, l_{1}^{\prime} b l_{3}, l_{1} l_{2}^{\prime} b\right\}$, where $l_{i} \in\left\{a, a^{\prime}\right\}$ for $i=1,2,3$, and $A=\left\{i_{1}<i_{2}<i_{3}\right\} \subseteq[d] \backslash\{i\}$. Observe that, since the words $v_{i^{c}}, u_{i^{c}}$ form a twin pair, the polybox code $V_{i^{c}}^{i, a} \cup V_{i^{c}}^{i, b^{\prime}}$, where $V^{i, b^{\prime}}=\{u\}$, does not contain a 
twin pair. Indeed, every word in $V_{i^{c}}^{i, a}$ contains at least two letters from the set $\left\{a, a^{\prime}\right\}$, while $u_{j} \in\left\{b, b^{\prime}\right\}$ for all $j \in[d] \backslash\{i\}$. Hence, by Lemma 16, the polybox code $V_{i^{c}}^{i, a} \cup V_{i^{c}}^{i, b^{\prime}}$ is rigid. Thus, by Lemma $5, V_{i^{c}}^{i, a} \cup V_{i^{c}}^{i, b^{\prime}}=W_{i^{c}}^{i, a} \cup W_{i^{c}}^{i, b^{\prime}}$. Since the codes $V$ and $W$ are disjoint, we have $V_{i^{c}}^{i, a}=W_{i^{c}}^{i, b^{\prime}}$. By $(\mathbf{C o})$ in Section 2.3, $V_{i^{c}}^{i, a} \sqsubseteq V_{i^{c}}^{i, a^{\prime}}$, and then, by Lemma $9,\left|V^{i, a^{\prime}}\right| \geqslant 5$, a contradiction.

Lemma 18. Let $V, W \subset S^{d}$ be disjoint sets which are equivalent polybox codes without twin pairs. If there are $i \in[d]$ and $l, s \in S, l \notin\left\{s, s^{\prime}\right\}$, such that $\left|V^{i, l}\right|=\left|V^{i, l^{\prime}}\right|=1$ and $\left|V^{i, s}\right| \neq\left|V^{i, s^{\prime}}\right|$ or $\left|V^{i, l}\right|=1$ and $2 \leqslant\left|V^{i, l^{\prime}}\right| \leqslant 4$, then $|V|>12$.

Proof. By Theorem 15, $|V| \geqslant 12$.

Let $\left|V^{i, l}\right|=\left|V^{i, l^{\prime}}\right|=1$. By Lemma $12, V^{i, l} \sqsubseteq W^{i, l}$ and $V^{i, l^{\prime}} \sqsubseteq W^{i, l^{\prime}}$, and thus, by Lemma $9,\left|W^{i, l}\right| \geqslant 5$ and $\left|W^{i, l^{\prime}}\right| \geqslant 5$.

Since $\left|V^{i, s}\right| \neq\left|V^{i, s^{\prime}}\right|$, we may assume, by $(\mathbf{V})$ in Section 2.3 , that $W^{i, s} \neq \emptyset$. Consequently, we may assume that $\left|W^{i, l}\right|=5$. Observe that, by Lemma 9 , the code $W^{i, l}$ is rigid.

Suppose now that for every $r \in S, r \notin\left\{l, l^{\prime}\right\}$, we have $W^{i, r}=\emptyset$ or $W^{i, r^{\prime}}=\emptyset$. Then, by Lemma 5 , there are $s_{1}, \ldots, s_{k} \in S, s_{n} \notin\left\{l, l^{\prime}, s_{m}, s_{m}^{\prime}\right\}$ for every $n, m \in[k], n \neq m$, such that $W_{i c}^{i, l}$ and $V_{i^{c}}^{i, l} \cup V_{i^{c}}^{i, s_{1}} \cup \cdots \cup V_{i^{c}}^{i, s_{k}}$ are equivalent. Since $W_{i^{c}}^{i, l}$ is rigid, we have $W_{i c}^{i, l}=V_{i^{c}}^{i, l} \cup V_{i^{c}}^{i, s_{1}} \cup \cdots \cup V_{i^{c}}^{i, s_{k}}$. Then $V^{i, l} \cap W^{i, l} \neq \emptyset$, which is a contradiction.

Therefore, there is $r \in S, r \notin\left\{l, l^{\prime}\right\}$ such that the sets $W^{i, r}$ and $W^{i, r^{\prime}}$ are non-empty. Clearly, it is enough to consider the case when $r=s$ because $W^{i, s} \neq \emptyset,\left|W^{i, l}\right|=5$ and $\left|W^{i, l^{\prime}}\right| \geqslant 5$. Suppose that $|V|=12$. Then $\left|W^{i, s}\right|=\left|W^{i, s^{\prime}}\right|=1$ and $\left|W^{i, l}\right|=\left|W^{i, l^{\prime}}\right|=5$. Observe that, by Lemma $12, W^{i, s} \sqsubseteq V^{i, s}$ and $W^{i, s^{\prime}} \sqsubseteq V^{i, s^{\prime}}$, which, by Lemma 9, implies that $\left|V^{i, s}\right| \geqslant 5,\left|V^{i, s^{\prime}}\right| \geqslant 5$. Since $\left|V^{i, s}\right| \neq\left|V^{i, s^{\prime}}\right|$, we have $V^{i, l}=\emptyset$ or $V^{i, l^{\prime}}=\emptyset$, a contradiction. Thus, $|V|>12$.

Let now $\left|V^{i, l}\right|=1$ and $2 \leqslant\left|V^{i, l^{\prime}}\right| \leqslant 4$. Obviously, $V^{i, s} \cup V^{i, s^{\prime}} \neq \emptyset$ for some $s \in S \backslash\left\{l, l^{\prime}\right\}$ because $|V| \geqslant 12$. If the codes $V_{i^{c}}^{i, s}$ and $V_{i^{c}}^{i, s^{\prime}}$ are equivalent, then, by Theorem 15, $|V|>\left|V^{i, s}\right|+\left|V^{i, s^{\prime}}\right| \geqslant 24$. Assume that $V_{i c}^{i, s}$ and $V_{i c^{i}}^{i, s^{\prime}}$ are not equivalent. Then, by $(\mathbf{P})$ in Section $2.3, W^{i, s} \cup W^{i, s^{\prime}} \neq \emptyset$. Since $\left|V^{i, l}\right|=1$ and $2 \leqslant\left|V^{i, l^{\prime}}\right| \leqslant 4$, by Lemma $12, V^{i, l} \sqsubseteq W^{i, l}$ and $V^{i, l^{\prime}} \sqsubseteq W^{i, l^{\prime}}$. Then, by Lemma 9 and $11,\left|W^{i, l}\right| \geqslant 5$ and $\left|W^{i, l^{\prime}}\right| \geqslant 7$, respectively. Thus, $|V|=|W| \geqslant\left|W^{i, l}\right|+\left|W^{i, l^{\prime}}\right|+\left|W^{i, s} \cup W^{i, s^{\prime}}\right|>12$ because $W^{i, s} \cup W^{i, s^{\prime}} \neq \emptyset$.

The following lemma will play an important role in determining the structure of the polybox codes $V$ and $W$.

Lemma 19. Let $S=\left\{a, a^{\prime}, b, b^{\prime}\right\}$, and let $V, W \subset S^{d}$ be disjoint sets which are equivalent polybox codes without twin pairs. Assume that there are $i \in[d]$ and $l, p \in S, l \neq p$, such that the sets $V^{i, l}$ and $V^{i, p}$ are non-empty. If there are letters $l_{1}, l_{2} \in S, l_{1} \notin\left\{l_{2}, l_{2}^{\prime}\right\}$, such that the polybox code $V^{i, l_{1}} \cup V^{i, l_{2}}$ does not contain $i$-siblings, then $|V|>12$.

Proof. By Theorem 15, $|V| \geqslant 12$. In the first part of the proof we show that the theorem is valid if $V^{i, s}=\emptyset$ for at least one $s \in S$. We will consider three cases.

In the first case we assume that $V^{i, s}=\emptyset$ or $V^{i, s^{\prime}}=\emptyset$ for every $s \in S$. Assume that $V^{i, s}=\emptyset$. Then, by Lemma 5 , the codes $V_{i^{c}}^{i, l}$ and $W_{i^{c}}^{i, l} \cup W_{i^{c}}^{i, s}$ are equivalent, where $s \notin\left\{l, l^{\prime}\right\}$. 
Note that $W^{i, s}=\emptyset$, for otherwise, by $(\mathbf{C o})$ in Section 2.3, $W_{i^{c}}^{i, s} \sqsubseteq V_{i^{c}}^{i, l^{\prime}}$, and thus $V^{i, l^{\prime}} \neq \emptyset$, which is a contradiction because $V^{i, l} \neq \emptyset$. Hence, the codes $V_{i^{c}}^{i, l}$ and $W_{i^{c}}^{i, l}$ are equivalent. Similarly, $V_{i^{c}}^{i, p}$ and $W_{i^{c}}^{i, p}$ are equivalent. By Theorem 15, $|V| \geqslant\left|V^{i, l}\right|+\left|V^{i, p}\right| \geqslant 24$.

In the second case we assume that $p=l^{\prime}$ and $V=V^{i, l} \cup V^{i, l^{\prime}}$. If $W=W^{i, l} \cup W^{i, l^{\prime}}$, then the polybox codes $V^{i, l}$ and $W^{i, l}$ are equivalent and similarly, $V^{i, l^{\prime}}$ and $W^{i, l^{\prime}}$ are equivalent. Then, by Theorem 15, $|V|=\left|V^{i, l}\right|+\left|V^{i, l^{\prime}}\right| \geqslant 24$.

If $W^{i, s} \neq \emptyset$, where $s \notin\left\{l, l^{\prime}\right\}$, then, by $(\mathbf{C})$ in Section 2.3, the set $\bigcup E\left(W^{i, s} \cup W^{i, s^{\prime}}\right)$ is an $i$-cylinder because $V^{i, s} \cup V^{i, s^{\prime}}=\emptyset$. Thus, the codes $W_{i^{c}}^{i, s}$ and $W_{i^{c}}^{i, s^{\prime}}$ are equivalent. By Theorem 15, $|W| \geqslant\left|W^{i, s}\right|+\left|W^{i, s^{\prime}}\right| \geqslant 24$, and hence $|V|>12$.

In the third case we assume that there is exactly one letter $s \in S$ such that $V^{i, s}=\emptyset$. Thus, we may assume that $V^{i, a} \neq \emptyset, V^{i, a^{\prime}} \neq \emptyset$ and $V^{i, b} \neq \emptyset, V^{i, b^{\prime}}=\emptyset$. Then, by Lemma 5 , the polybox codes $V_{i^{c}}^{i, a}$ and $W_{i^{c}}^{i, a} \cup W_{i^{c}}^{i, b^{\prime}}$ are equivalent. Similarly, $V_{i^{c}}^{i, a^{\prime}}$ and $W_{i^{c}}^{i, a^{\prime}} \cup W_{i^{c}}^{i, b^{\prime}}$ are equivalent.

If $V^{i, a}$ is not rigid, then, by Lemma $16,\left|V^{i, a}\right| \geqslant 10$. Thus, only the case $\left|V^{i, a}\right|=10$, $\left|V^{i, a^{\prime}}\right|=1$ and $\left|V^{i, b}\right|=1$ has to be considered. Since $V^{i, b^{\prime}}=\emptyset$, by $(\mathbf{P}), V^{i, b} \sqsubseteq W^{i, b}$, and consequently, by Lemma $9,\left|W^{i, b}\right| \geqslant 5$. Note that, by $(\mathbf{V}),\left|W^{i, a}\right| \geqslant 9$ because $\left|V^{i, a}\right|=10$ and $\left|V^{i, a^{\prime}}\right|=1$. Therefore, $|W|>\left|W^{i, a}\right|+\left|W^{i, b}\right| \geqslant 14$, which contradicts the assumption that $|V|=12$. Thus, $|V|>12$.

If the codes $V^{i, a}$ and $V^{i, a^{\prime}}$ are rigid, then $V_{i^{c}}^{i, a}=W_{i^{c}}^{i, a} \cup W_{i^{c}}^{i, b^{\prime}}$ and $V_{i^{c}}^{i, a^{\prime}}=W_{i^{c}}^{i, a^{\prime}} \cup W_{i^{c}}^{i, b^{\prime}}$. Since $V \cap W=\emptyset$, the set $W^{i, a} \cup W^{i, a^{\prime}}$ is empty. Then, by (C) in Section 2.3, the set $\bigcup E\left(V^{i, a} \cup V^{i, a^{\prime}}\right)$ is an $i$-cylinder. Consequently, the codes $V_{i^{c}}^{i, a}$ and $V_{i^{c}}^{i, a^{\prime}}$ are equivalent, and since they are rigid, we have $V_{i^{c}}^{i, a}=V_{i^{c}}^{i, a^{\prime}}$. Hence, the set $V^{i, a} \cup V^{i, a^{\prime}}$ contains a twin pair, which is a contradiction. This completes the first part of the proof.

In the second part of the proof we assume that $V^{i, s} \neq \emptyset$ for every $s \in S$.

Suppose that the set $V^{i, a} \cup V^{i, b}$ does not contain $i$-siblings (we assume that $l_{1}=a$ and $\left.l_{2}=b\right)$. Then $V_{i^{c}}^{i, a} \cup V_{i^{c}}^{i, b}$ does not contain a twin pair.

If $\left|V^{i, a} \cup V^{i, b}\right| \geqslant 10$, then assume that $\left|V^{i, a} \cup V^{i, b}\right|=10$ and $\left|V^{i, a^{\prime}}\right|=\left|V^{i, b^{\prime}}\right|=1$. By Lemma 18, $\left|V^{i, a}\right|=5,\left|V^{i, a^{\prime}}\right|=1$ and $\left|V^{i, b}\right|=5,\left|V^{i, b^{\prime}}\right|=1$, which is, by Lemma 17, impossible. Thus, $|V|>12$.

If $\left|V^{i, a} \cup V^{i, b}\right| \leqslant 9$ then, by Lemma 16 , the polybox code $V_{i c}^{i, a} \cup V_{i c}^{i, b}$ is rigid. By Lemma 5, the codes $V_{i^{c}}^{i, a} \cup V_{i^{c}}^{i, b}$ and $W_{i^{c}}^{i, a} \cup W_{i^{c}}^{i, b}$ are equivalent. Thus, $V_{i^{c}}^{i, a} \cup V_{i^{c}}^{i, b}=W_{i^{c}}^{i, a} \cup W_{i^{c}}^{i, b}$. Consequently, $V_{i^{c}}^{i, a}=W_{i^{c}}^{i, b}$ and $V_{i^{c}}^{i, b}=W_{i^{c}}^{i, a}$ because $V$ and $W$ are disjoint. Therefore, by (Co) in Section 2.3, $V_{i^{c}}^{i, a} \sqsubseteq V_{i^{c}}^{i, a^{\prime}}$ and $V_{i^{c}}^{i, b} \sqsubseteq V_{i^{c}}^{i, b^{\prime}}$. By Lemma 9, $\left|V^{i, a^{\prime}}\right| \geqslant 5,\left|V^{i, b^{\prime}}\right| \geqslant 5$. Since the sets $V^{i, a}$ and $V^{i, b}$ are non-empty and the distribution $\left|V^{i, a^{\prime}}\right|=5,\left|V^{i, a}\right|=1$ and $\left|V^{i, b^{\prime}}\right|=5,\left|V^{i, b}\right|=1$ is, by Lemma 17 , impossible, it follows that $|V|>12$.

From Lemmas 18 and 19 we obtain the following corollary which will be used in the computations.

Corollary 20. Let $S=\left\{a, a^{\prime}, b, b^{\prime}\right\}, V, W \subset S^{d}$ be disjoint sets which are equivalent polybox codes without twin pairs, and let

$$
R=\{(6,5),(6,6),(7,2),(7,3),(7,4),(7,5),(8,0),(8,1),(8,2),(8,3),(8,4),
$$




$$
(9,0),(9,1),(9,2),(9,3),(10,0),(10,1),(10,2),(11,0),(11,1)\} .
$$

Suppose that there are $i \in[d]$ and $s \in S$ such that $\left(\left|V^{i, s}\right|,\left|V^{i, s^{\prime}}\right|\right) \in R$. Then $|V|>12$.

Proof. By Theorem 15, $|V| \geqslant 12$. Thus, by the assumption on $V^{i, s}$ and $V^{i, s^{\prime}}$, there are two letters $l, p \in S$ such that $V^{i, l}$ and $V^{i, p}$ are non-empty sets. Suppose that there is a letter in the set $S$, say it is the letter $a$, such that $V^{i, a}=\emptyset$. Then there are no two words $v, u \in V$ which are $i$-siblings such that $\left\{v_{i}, u_{i}\right\} \in\left\{\{a, b\},\left\{a, b^{\prime}\right\}\right\}$. By Lemma 19, $|V|>12$.

Assume now that all sets $V^{i, a}, V^{i, a^{\prime}}, V^{i, b}$ and $V^{i, b^{\prime}}$ are non-empty and suppose on the contrary that $|V|=12$. Assume that $s=a$. Then $\left(\left|V^{i, b}\right|,\left|V^{i, b^{\prime}}\right|\right) \in\{(1,1),(1,2)\}$. By Lemma 18, $|V|>12$, a contradiction.

In the last lemma we show that the polybox codes $V$ and $W$ can be written down in the alphabet $S=\left\{a, a^{\prime}, b, b^{\prime}\right\}$.

Lemma 21. Let $V, W \subset S^{d}$ be disjoint sets which are equivalent polybox codes without twin pairs. If there are $i \in[d]$ and $l_{1}, l_{2}, l_{3} \in S$, where $l_{1} \notin\left\{l_{2}, l_{2}^{\prime}, l_{3}, l_{3}^{\prime}\right\}, l_{2} \notin\left\{l_{3}, l_{3}^{\prime}\right\}$, such that $V^{i, l_{k}} \cup V^{i, l_{k}^{\prime}} \neq \emptyset$ for $k=1,2,3$, then $|V|>12$. Moreover, if $|V|=12$, then the polybox codes $V, W$ might be written down in the alphabet $\left\{a, a^{\prime}, b, b^{\prime}\right\}$.

Proof. It follows from Theorem 15 that $|V| \geqslant 12$. To prove the first part of the lemma, let us suppose on the contrary that $|V|=12$. Assume that $l_{1}=a, l_{2}=b$ and $l_{3}=c$. Note that

$$
W^{i, l} \cup W^{i, l^{\prime}} \neq \emptyset
$$

for every $l \in\{a, b, c\}$. Indeed, if $W^{i, l} \cup W^{i, l^{\prime}}=\emptyset$ for some $l \in\{a, b, c\}$, then, by (C) in Section 2.3, the set $\bigcup E\left(V^{i, l} \cup V^{i, l^{\prime}}\right)$ is an $i$-cylinder and consequently the codes $V_{i^{i}}^{i, l}$ and $V_{i c^{i}}^{i, l^{\prime}}$ are equivalent. By Theorem $15,\left|V^{i, l}\right|+\left|V^{i, l^{\prime}}\right| \geqslant 24$, a contradiction.

We will show that $V^{i, l} \neq \emptyset$ for every $l \in\left\{a, a^{\prime}, b, b^{\prime}, c, c^{\prime}\right\}$.

Assume that there is a letter in the set $\{a, b, c\}$, say it is the letter $c$, such that $V^{i, c} \neq \emptyset$ and $V^{i, c^{\prime}}=\emptyset$. Then, by $(\mathbf{P})$ in Section 2.3., $V^{i, c} \sqsubseteq W^{i, c}$, and thus, by Lemma $9,\left|W^{i, c}\right| \geqslant 5$. If $\left|W^{i, a} \cup W^{i, a^{\prime}}\right| \geqslant 4$ and $\left|W^{i, b} \cup W^{i, b^{\prime}}\right| \geqslant 4$, then $|W|>12$, a contradiction. Thus, we may assume that $\left|W^{i, b} \cup W^{i, b^{\prime}}\right| \leqslant 3$, and then, by Lemma 18, we may assume that $W^{i, b} \neq \emptyset$ and $W^{i, b^{\prime}}=\emptyset$ or $\left|W^{i, b}\right|=\left|W^{i, b^{\prime}}\right|=1$. We now consider these two cases in detail.

Let $W^{i, b} \neq \emptyset$ and $W^{i, b^{\prime}}=\emptyset$. By $(\mathbf{P}), W^{i, b} \sqsubseteq V^{i, b}$ and then $\left|V^{i, b}\right| \geqslant 5$, by Lemma 9 .

If $W^{i, c^{\prime}}=\emptyset$, then, by $(\mathbf{P}), W^{i, c} \sqsubseteq V^{i, c}$. Since $V^{i, c} \sqsubseteq W^{i, c}$, the codes $V^{i, c}$ and $W^{i, c}$ are equivalent. By Theorem 15, $\left|V^{i, c}\right| \geqslant 12$. Hence, $|V|>\left|V^{i, b}\right|+\left|V^{i, c}\right|>12$, a contradiction.

Therefore, $W^{i, c^{\prime}} \neq \emptyset$. Then $\left|W^{i, c} \cup W^{i, c^{\prime}}\right| \geqslant 6$ because $\left|W^{i, c}\right| \geqslant 5$. Since $V^{i, c^{\prime}}=\emptyset$, by $(\mathbf{P})$, we have $W_{i^{c}}^{i, c^{\prime}} \sqsubseteq W_{i^{c}}^{i, c}$. By Lemma 11, if $\left|W_{i^{c}}^{i, c^{\prime}}\right| \geqslant 2$, then $\left|W_{i^{c}}^{i, c}\right| \geqslant 7$. Clearly, $\left|W^{i, c} \cup W^{i, c^{\prime}}\right|<11$ because (4.2) and $|V|=12$. Thus, $\left|W_{i^{c}}^{i, c^{\prime}}\right| \leqslant 3$. Then, by $(\mathbf{P})$ and $(\mathbf{V})$ in Section 2.3, $\left|V^{i, c}\right| \geqslant 4$. Hence, $\left|V^{i, a} \cup V^{i, a^{\prime}}\right| \leqslant 3$ as $\left|V^{i, b}\right| \geqslant 5$. By Lemma 18, we may assume that $V^{i, a} \neq \emptyset$ and $V^{i, a^{\prime}}=\emptyset$ or $\left|V^{i, a}\right|=\left|V^{i, a^{\prime}}\right|=1$.

In the first case we have $\left|W^{i, a}\right| \geqslant 5$, by $(\mathbf{P})$ and Lemma 9. Note that $W^{i, a^{\prime}} \neq \emptyset$, for otherwise $W^{i, a} \sqsubseteq V^{i, a}$, and then $\left|V^{i, a}\right| \geqslant 7$. Consequently, $V^{i, c} \cup V^{i, c^{\prime}}=\emptyset$ because 
$\left|V^{i, b}\right| \geqslant 5$, a contradiction. Since $\left|W^{i, c} \cup W^{i, c^{\prime}}\right| \geqslant 6,\left|W^{i, a}\right| \geqslant 5$ and $W^{i, a^{\prime}} \neq \emptyset$, it follows that $W^{i, b} \cup W^{i, b^{\prime}}=\emptyset$, a contradiction.

Let $\left|V^{i, a}\right|=\left|V^{i, a^{\prime}}\right|=1$. Since $\left|V^{i, c}\right| \neq\left|V^{i, c^{\prime}}\right|$, by Lemma 18, $|V|>12$. A contradiction.

Let now $\left|W^{i, b}\right|=\left|W^{i, b^{\prime}}\right|=1$. By Lemma 18 , it must be $\left|W^{i, c}\right|=\left|W^{i, c^{\prime}}\right|$, for otherwise $|W|>12$, which contradicts the assumption $|V|=12$. Since $\left|W^{i, c}\right| \geqslant 5$, we have $W^{i, a} \cup$ $W^{i, a^{\prime}}=\emptyset$, a contradiction.

We have shown that the sets $V^{i, l}, V^{i, l^{\prime}}$ are non-empty for every $l \in\{a, b, c\}$. Clearly, in the same way we show that the sets $W^{i, l}, W^{i, l^{\prime}}$ are non-empty for every $l \in\{a, b, c\}$.

Thus, if $\left|V^{i, c} \cup V^{i, c^{\prime}}\right| \leqslant 3$, then, by Lemma 18, $\left|V^{i, c}\right|=1$ and $\left|V^{i, c^{\prime}}\right|=1$. By Lemma $12, V^{i, c} \sqsubseteq W^{i, c}$ and $V^{i, c^{\prime}} \sqsubseteq W^{i, c^{\prime}}$ and consequently $\left|W^{i, c}\right| \geqslant 5$ and $\left|W^{i, c^{\prime}}\right| \geqslant 5$, by Lemma 9. This means that $W^{i, l}=\emptyset$ for some $l \in\left\{a, a^{\prime} b, b^{\prime}\right\}$, which is a contradiction. Hence, $\left|V^{i, l} \cup V^{i, l^{\prime}}\right|=4$, and by Lemma 18,

$$
\left|V^{i, l}\right|=\left|V^{i, l^{\prime}}\right|=2
$$

for $l \in\{a, b, c\}$.

Observe now that for every $l \in\{a, b, c\}$ there is $w \in W$ such that $\breve{w} \cap \bigcup E\left(V^{i, l}\right) \neq \emptyset$ and $\breve{w} \cap \bigcup E\left(V^{i, l^{\prime}}\right) \neq \emptyset$, for otherwise $V^{i, l} \sqsubseteq W^{i, l}$ and $V^{i, l^{\prime}} \sqsubseteq W^{i, l^{\prime}}$ for some $l \in\{a, b, c\}$, and thus, by Lemma $11,\left|W^{i, l}\right| \geqslant 7$ and $\left|W^{i, l^{\prime}}\right| \geqslant 7$. Then $|W|>12$, a contradiction.

Therefore, for every $l \in\{a, b, c$,$\} the form of the polybox code V^{i, l} \cup V^{i, l^{\prime}}$ is as described in Lemma 14. In the next two steps, which are $d=4$ and $d \geqslant 5$, we show that this is impossible.

Let $d=4$. For simplicity assume that $i=1$ and the form of $V^{1, a} \cup V^{1, a^{\prime}}$ is as at the first four positions in Table 1 (compare also Example 4). Let $w \in W$ be such that $\breve{w} \cap \bigcup E\left(V^{1, a}\right) \neq \emptyset$ and $\breve{w} \cap \bigcup E\left(V^{1, a^{\prime}}\right) \neq \emptyset$. Thus, $w_{1} \notin\left\{a, a^{\prime}\right\}$. We may assume that $w_{1}=b$. Clearly, $w_{2}=w_{3}=b$, by Lemma 14 .

Pick $x \in \bigcup E\left(V^{1, a} \cup V^{1, a^{\prime}}\right)$ such that $x_{1} \in E b^{\prime}, x_{2}, x_{3} \in E b$ and $x_{4} \in E a \cap E w_{4}$. The point $x_{1^{c}}$ belongs to $\breve{w}_{1^{c}}$ and therefore, $x$ has to be covered by a box $\breve{u}$ for some $u \in W^{1, b^{\prime}}$. Since $x$ belongs to $\bigcup E\left(V^{1, a} \cup V^{1, a^{\prime}}\right)$ and $u_{1}=b^{\prime}$, we have $\breve{u} \cap \bigcup E\left(V^{1, a}\right) \neq \emptyset$ and $\breve{u} \cap \bigcup E\left(V^{1, a^{\prime}}\right) \neq \emptyset$. Then, $u_{2}=u_{3}=b$, by Lemma 14 . Therefore, $w_{4} \neq u_{4}$, for otherwise $w$ and $u$ form a twin pair, which is impossible.

We first assume that $w_{4} \neq a$. Then, by (2.3), $E a \cap E u_{4} \backslash E w_{4} \neq \emptyset$ (we have $u_{4} \neq a^{\prime}$ because $\left.\breve{u} \cap \bigcup E\left(V^{i, a} \cup V^{1, a^{\prime}}\right) \neq \emptyset\right)$. We pick a point $y \in \bigcup E\left(V^{1, a} \cup V^{1, a^{\prime}}\right)$ such that $y_{1} \in E b, y_{2}, y_{3} \in E b$ and $y_{4} \in E a \cap E u_{4} \backslash E w_{4}$. Then $y \notin \breve{w} \cup \breve{u}$. For the same reason as above, the point $y$ is covered by a box $\breve{v}$ for some word $v \in W^{1, b}$ with $v_{2}=v_{3}=b$. Since $v_{4^{c}}=w_{4^{c}}$ and $v, w$ are dichotomous words, $v_{4}^{\prime}=w_{4}$. Then $v$ and $w$ are a twin pair, a contradiction.

Let $w_{4}=a$. Now, we pick a point $z \in \bigcup E\left(V^{1, a} \cup V^{1, a^{\prime}}\right)$ such that $z_{1} \in E b^{\prime}, z_{2}, z_{3} \in E b$ and $z_{4} \in E a \backslash E u_{4}$. Then $z \notin \breve{w} \cup \breve{u}$. The point $z$ is covered by a box $\breve{q}$ for some $q \in W^{1, b^{\prime}}$ with $q_{2}=q_{3}=b$. Since $u_{4^{c}}=q_{4^{c}}$ and $u, q$ are dichotomous words, $u_{4}^{\prime}=q_{4}$. Then $u$ and $q$ are a twin pair, a contradiction.

The proof of the first part of the lemma for $d=4$ is completed.

Let now $d \geqslant 5$. We may assume without loss of generality that $i=1$ and the form of $V^{1, a} \cup V^{1, a^{\prime}}$ at the first five position is, by Lemma 14, as presented in Table 1 . 
The distributions of words in the polybox codes $V^{1, b} \cup V^{1, b^{\prime}}$ and $V^{1, c} \cup V^{1, c^{\prime}}$ at positions $2, \ldots, d$ are analogous to the distributions of words in $V^{1, a} \cup V^{1, a^{\prime}}$ but the positions of the corresponding columns in these two codes as well as the letters in the columns may be different from those in $V^{1, a} \cup V^{1, a^{\prime}}$. Thus, we may assume that there are $j \in\{4,5\}$ and letters $l, s, r, p \in S$ such that

$$
\left|\left(V^{1, a} \cup V^{1, a^{\prime}}\right)^{j, l}\right|=4, \quad\left|\left(V^{1, b} \cup V^{1, b^{\prime}}\right)^{j, s}\right|=4, \quad\left|\left(V^{1, c} \cup V^{1, c^{\prime}}\right)^{j, r}\right|=4
$$

or

$$
\begin{gathered}
\left|\left(V^{1, a} \cup V^{1, a^{\prime}}\right)^{j, l}\right|=4, \quad\left|\left(V^{1, b} \cup V^{1, b^{\prime}}\right)^{j, s}\right|=4, \\
\left|\left(V^{1, c} \cup V^{1, c^{\prime}}\right)^{j, p}\right|=2, \quad\left|\left(V^{1, c} \cup V^{1, c^{\prime}}\right)^{j, p^{\prime}}\right|=1, \quad\left|\left(V^{1, c} \cup V^{1, c^{\prime}}\right)^{j, r}\right|=1,
\end{gathered}
$$

where $r \notin\left\{p, p^{\prime}\right\}$.

\begin{tabular}{|c|c|c|c|c|c|}
\hline$V^{1, a} \cup V^{1, a^{\prime}}$ & $i=1$ & $i=2$ & $i=3$ & $i=4$ & $i=5$ \\
\hline$v^{1}$ & $a$ & $a^{\prime}$ & $b$ & $a$ & $a$ \\
$v^{2}$ & $a$ & $a$ & $a$ & $a$ & $a$ \\
$v^{3}$ & $a^{\prime}$ & $a^{\prime}$ & $a^{\prime}$ & $a$ & $a$ \\
$v^{4}$ & $a^{\prime}$ & $b$ & $a$ & $a$ & $a$ \\
\hline
\end{tabular}

Table 1.

We first consider the distribution (4.4). There are five cases that have to be examined.

The first case is $l=s=r$, and thus $V=V^{j, l}$, where $\left|V^{j, l}\right|=12$. Then, by induction on $d$, we obtain $\left|V_{j^{c}}\right|>12$, a contradiction.

The second case is $l \notin\left\{s, s^{\prime}, r, r^{\prime}\right\}, s \notin\left\{r, r^{\prime}\right\}$, and thus $V=V^{j, l} \cup V^{j, s} \cup V^{j, r}$, where $\left|V^{j, l}\right|=\left|V^{j, s}\right|=\left|V^{j, r}\right|=4$. By $(\mathbf{P}), W=W^{j, l} \cup W^{j, s} \cup W^{j, r}$. Therefore, the codes $V^{j, l}$ and $W^{j, l}$ are equivalent. By Lemma 9, the polybox code $V^{j, l}$ is rigid because it does not cover even a single word. Hence, $V^{j, l}=W^{j, l}$, and then $V \cap W \neq \emptyset$, which is a contradiction.

Similarly, in the third case $r=s$ and $l \notin\left\{s, s^{\prime}\right\}$, that is, $V=V^{j, l} \cup V^{j, s}$, where $\left|V^{j, l}\right|=4$ and $\left|V^{j, s}\right|=8$, we obtain $V \cap W \neq \emptyset$, which is not possible.

In the fourth case we have $l^{\prime}=s$ and $r \notin\left\{l, l^{\prime}\right\}$. Then $V=V^{j, l} \cup V^{j, l^{\prime}} \cup V^{j, r}$, where $\left|V^{j, l}\right|=\left|V^{j, l^{\prime}}\right|=\left|V^{j, r}\right|=4$. By Lemma 5, there are $s_{1}, \ldots, s_{k} \in S, s_{n} \notin\left\{l, l^{\prime}, s_{m}, s_{m}^{\prime}\right\}$ for every $n, m \in[k], n \neq m$, such that $V_{i^{c}}^{i, l}$ and $W_{i^{c}}^{i, l} \cup W_{i^{c}}^{i, s_{1}} \cup \cdots \cup W_{i^{c}}^{i, s_{k}}$ are equivalent. Since, by Lemma 9, the code $V^{j, l}$ is rigid, we have $V_{i^{c}}^{i, l}=W_{i^{c}}^{i, l} \cup W_{i^{c}}^{i, s_{1}} \cup \cdots \cup W_{i^{c}}^{i, s_{k}}$. The codes $V$ and $W$ are disjoint, and therefore $W^{i, l}=\emptyset$. Then $V_{i^{c}}^{i, l}=W_{i^{c}}^{i, s_{1}} \cup \cdots \cup W_{i^{c}}^{i, s_{k}}$. By $(\mathbf{C})$ in Section 2.3, $W_{i^{c}}^{i, s_{1}} \cup \cdots \cup W_{i^{c}}^{i, s_{k}} \sqsubseteq V_{i^{c}}^{i, l^{\prime}}$ and, by Lemma $11,\left|V^{i, l^{\prime}}\right| \geqslant 7$, a contradiction.

Finally, in the fifth case we have $l=s$ and $r=l^{\prime}$. Then $V=V^{j, l} \cup V^{j, l^{\prime}}$. We may assume that $\left|V^{j, l}\right|=8$ and $\left|V^{j, l^{\prime}}\right|=4$. By Lemma 9, the code $V^{j, l^{\prime}}$ is rigid. Therefore, if $W=W^{j, l} \cup W^{j, l^{\prime}}$, then $V^{j, l^{\prime}}=W^{j, l^{\prime}}$. Hence, $V$ and $W$ are not disjoint, which is not true. Thus, $W^{j, p} \cup W^{j, p^{\prime}} \neq \emptyset$ for some $p \notin\left\{l, l^{\prime}\right\}$. By $(\mathbf{C})$ in Section 2.3, the set 
$\bigcup E\left(W^{j, p} \cup W^{j, p^{\prime}}\right)$ is a $j$-cylinder. Then, the codes $W_{j^{c}}^{j, p}$ and $W_{j^{c}}^{j, p^{\prime}}$ are equivalent. By Theorem 15, $|W| \geqslant\left|W^{j, p}\right|+\left|W^{j, p^{\prime}}\right| \geqslant 24$, a contradiction.

To consider the distribution (4.5) observe first that $p \in\left\{l, l^{\prime}, s, s^{\prime}\right\}$, for otherwise, by Lemma $18,|V|>12$, which contradicts our assumption on $|V|$.

Similarly, $r \in\left\{l, l^{\prime}, s, s^{\prime}\right\}$, for otherwise $V^{j, r} \sqsubseteq W^{j, r}$ and, by Lemma $9,\left|W^{j, r}\right| \geqslant 5$. Since $\left|V^{j, r}\right|=1$, we have, by $(\mathbf{V}),\left|W^{j, r}\right|-\left|W^{j, r^{\prime}}\right| \leqslant 1$. Then $\left|W^{j, r^{\prime}}\right| \geqslant 4$. Note that $W_{j^{c}}^{j, r^{\prime}} \sqsubseteq W_{j^{c}}^{j, r}$, for otherwise, by $(\mathbf{P}), V^{j, r^{\prime}} \neq \emptyset$, which is not true. Therefore, by Lemma $11,\left|W^{j, r}\right| \geqslant 7$ and consequently $\left|W^{j, r^{\prime}}\right| \geqslant 6$. Then $|W|>12$, a contradiction.

Thus, in the rest part of the proof we assume that $l \in\left\{r, r^{\prime}\right\}$ and $s \in\left\{p, p^{\prime}\right\}$ (recall that $r \notin\left\{p, p^{\prime}\right\}$ ). Hence, only two cases have to be considered: $\left|V^{j, r}\right|=5, V^{j, r^{\prime}}=\emptyset$ (and then $\left|V^{j, p}\right|=6,\left|V^{j, p^{\prime}}\right|=1$ or $\left.\left|V^{j, p}\right|=2,\left|V^{j, p^{\prime}}\right|=5\right)$ and $\left|V^{j, r}\right|=1,\left|V^{j, r^{\prime}}\right|=4$.

Let $\left|V^{j, r}\right|=5, V^{j, r^{\prime}}=\emptyset$. Then, by $(\mathbf{P}), V^{j, r} \sqsubseteq W^{j, r}$ and thus, by Lemma 11, $\left|W^{j, r}\right| \geqslant 7$. By $(\mathbf{V}),\left|W^{j, r^{\prime}}\right| \geqslant 2$ because $\left|V^{j, r}\right|=5$.

If $\left|V^{j, p}\right|=6,\left|V^{j, p^{\prime}}\right|=1$, then, by $(\mathbf{V}),\left|W^{j, p}\right| \geqslant 5$, and consequently $|W| \geqslant\left|W^{j, r}\right|+$ $\left|W^{j, r^{\prime}}\right|+\left|W^{j, p}\right| \geqslant 14$, a contradiction.

If $\left|V^{j, p}\right|=2,\left|V^{j, p^{\prime}}\right|=5$, then, by Lemma 11, $V_{j^{c}}^{j, p} \nsubseteq V_{j^{c}}^{j, p^{\prime}}$. Therefore, $\bigcup E\left(V_{j^{c}}^{j, p}\right) \backslash$ $\bigcup E\left(V_{j^{c}}^{j, p^{\prime}}\right) \neq \emptyset$ and then, by $(\mathbf{P}), W^{j, p} \neq \emptyset$. Moreover, by $(\mathbf{V}),\left|W^{j, p^{\prime}}\right| \geqslant 3$. Then $|W| \geqslant\left|W^{j, r}\right|+\left|W^{j, r^{\prime}}\right|+\left|W^{j, p}\right|+\left|W^{j, p^{\prime}}\right| \geqslant 13$, a contradiction.

Let $\left|V^{j, r}\right|=1,\left|V^{j, r^{\prime}}\right|=4$. Then, by Lemma $18,|V|>12$, a contradiction. This completes the proof of the first part of the lemma.

Thus, if $|V|=12$, then for every $i \in[d]$ there are at most two letters $l, s \in S, l \notin\left\{s, s^{\prime}\right\}$ such that the sets $V^{i, l} \cup V^{i, l^{\prime}}$ and $V^{i, s} \cup V^{i, s^{\prime}}$ are non-empty. Since there are no connection between the letters standing at the two different positions $i, j \in[d]$ in the code $V$, we may assume that $V \subset\left\{a, a^{\prime}, b, b^{\prime}\right\}^{d}$.

To show that $W \subset\left\{a, a^{\prime}, b, b^{\prime}\right\}^{d}$ assume on the contrary that there is $i \in[d]$ such that $W^{i, l} \cup W^{i, l^{\prime}} \neq \emptyset$, where $l \notin\left\{a, a^{\prime}, b, b^{\prime}\right\}$. Since $V^{i, l} \cup V^{i, l^{\prime}}=\emptyset$ and $W^{i, l} \cup W^{i, l^{\prime}} \neq \emptyset$, by $(\mathbf{C})$ in Section 2.3, the set $\bigcup E\left(W^{i, l} \cup W^{i, l^{\prime}}\right)$ is an $i$-cylinder. Consequently, by Theorem 15, $|W| \geqslant\left|W^{i, l}\right|+\left|W^{i, l^{\prime}}\right| \geqslant 24$, a contradiction.

\section{Computations}

In this section we describe the computations that allow us to determine all equivalent and disjoint polybox codes $V, W \subset S^{d}$ without twin pairs having 12 words each, where $S=\left\{a, a^{\prime}, b, b^{\prime}\right\}$ and $d \in\{4,5,6\}$.

Before we start let us recall the definition of isomorphic polybox codes.

If $v \in S^{d}$, and $\sigma$ is a permutation of the set $[d]$, then $\sigma^{*}(v)=v_{\sigma(1)} \ldots v_{\sigma(d)}$. For every $i \in[d]$ let $h_{i}: S \rightarrow S$ be a bijection such that $h_{i}\left(l^{\prime}\right)=\left(h_{i}(l)\right)^{\prime}$ for every $l \in S$, and let $h: S^{d} \rightarrow S^{d}$ be defined by the formula $h(v)=h_{1}\left(v_{1}\right) \ldots h_{d}\left(v_{d}\right)$. We say that polybox codes $P, Q \subset S^{d}$ are isomorphic if there are $\sigma$ and $h$ such that $Q=\left\{h_{1}\left(v_{\sigma(1)}\right) \ldots h_{d}\left(v_{\sigma(d)}\right)\right.$ : $v \in P\}$. The composition $h \circ \sigma^{*}$ is an isomorphism between $P$ and $Q$. Let $V$ and $W$ be disjoint, equivalent and twin pair free polybox codes, and let $h \circ \sigma^{*}(V)$ be an isomorphic code to $V$. It follows from the definition of the isomorphism $h \circ \sigma^{*}$ that the codes $h \circ \sigma^{*}(V)$ 
and $h \circ \sigma^{*}(W)$ are also disjoint, equivalent and do not contain a twin pair. To show this it is enough to notice that the definition of $h \circ \sigma^{*}$ guarantees that $h \circ \sigma^{*}(w) \sqsubseteq h \circ \sigma^{*}(V)$, whenever $w \sqsubseteq V$.

\subsection{Covers of a pair of words}

We begin with the following

Lemma 22. Let $S=\left\{a, a^{\prime}, b, b^{\prime}\right\}, d \in\{4,5,6\}$, and let $V, W \subset S^{d}$ be disjoint sets which are equivalent polybox codes without twin pairs. Assume that for every $i \in[d]$ there are two letters $l, p \in S$ such that $W^{i, l} \neq \emptyset$ and $W^{i, p} \neq \emptyset$. If $|V|=12$, then for every $v \in V$ the number of words in the set $W_{v}=\{w \in W: \breve{w} \cap \breve{v} \neq \emptyset\}$ is at most eight for $d=4$, seven for $d=5$ and six for $d=6$.

Proof. We prove the lemma for $d=6$. Suppose on the contrary that $\left|W_{v}\right|=7$. Assume for simplicity that $v=b b b b b b$. For every $s \in S$ we have $E s \cap E s^{\prime}=\emptyset$ and therefore every word in $W_{v}$ has no letter $b^{\prime}$ and every word in $W \backslash W_{v}$ has $b^{\prime}$ at some position $i \in[6]$. Since $|W|=$ 12 , by Lemma 19, for every $i \in[6]$ and every pair $\{l, s\} \in\left\{\{a, b\},\left\{a, b^{\prime}\right\},\left\{a^{\prime}, b\right\},\left\{a^{\prime}, b^{\prime}\right\}\right\}$ there are two words $p, q \in W$ which are $i$-siblings such that $\left\{p_{i}, q_{i}\right\}=\{l, s\}$. We will consider a graph $G$ of siblings on $W$ (compare Section 2.9). Call an edge $(p, q)$ in $G$ of the type $b^{\prime}$ if $\left\{p_{i}, q_{i}\right\} \in\left\{\left\{a, b^{\prime}\right\},\left\{a^{\prime}, b^{\prime}\right\}\right\}$. Since vertices in $W_{v}$ do not contain the letter $b^{\prime}$, both endpoints of any edge of the type $b^{\prime}$ belong to the set $W \backslash W_{v}$ (call such an edge an inner edge) or one of the endpoints lies in $W_{v}$ and the other in $W \backslash W_{v}$ (call such an edge an outer edge) .

Clearly, since vertices in $W_{v}$ do not contain the letter $b^{\prime}$, if $w \in W \backslash W_{v}$ contains at least three letters $b^{\prime}$, then there is no outer edge which is incident to $w$.

Observe that, if $w \in W \backslash W_{v}$ contains two letters $b^{\prime}$, then there is at most one outer edge which is incident to $w$. To see this, assume that $w=b^{\prime} b^{\prime} w_{3} w_{4} w_{5} w_{6}$ and suppose on the contrary that there are two outer edges, say $(w, u)$ and $(w, p)$, where $u, p \in W_{v}$. We may assume that $u=a b w_{3} w_{4} w_{5} w_{6}$. Then $p=a^{\prime} b w_{3} w_{4} w_{5} w_{6}, p=b a w_{3} w_{4} w_{5} w_{6}$ or $p=b a^{\prime} w_{3} w_{4} w_{5} w_{6}$. In the first case $u$ and $p$ form a twin pair, and in the rest two cases $u$ and $p$ are not dichotomous. In both cases we obtain a contradiction.

Recall that the outer and the inner edges in $G$ are coloured with the colours $\{1, \ldots, 6\}$ : An edge $(p, q)$ of the type $b^{\prime}$ has a colour $i \in[6]$ if $\left\{p_{i}, q_{i}\right\} \in\left\{\left\{a, b^{\prime}\right\},\left\{a^{\prime}, b^{\prime}\right\}\right\}$. We need at least two edges of each colour $i \in[6]$ : One edge $(p, q)$ with $\left\{p_{i}, q_{i}\right\}=\left\{a, b^{\prime}\right\}$ and the second one $(u, t)$ with $\left\{u_{i}, t_{i}\right\}=\left\{a^{\prime}, b^{\prime}\right\}$.

Let $\left\{w^{1}, \ldots, w^{5}\right\}=W \backslash W_{v}$. In Figures $13 a, b$, we indicate the forbidden colourings of subgraphs of $G$, where $i, j, k \in[6]$ are pairwise different and all edges are of the type $b^{\prime}$ (slanted edges in Figures 13a, $b$ are outer edges).

To show that the colouring in Figure $13 a$ is forbidden assume on the contrary that $i=1, j=2, k=3$. Then we may assume that $w^{n}=b^{\prime} w_{2}^{n} \ldots w_{6}^{n}$, where $w_{i}^{n} \neq b^{\prime}$ for $i=2, \ldots, 6$, because $w^{n}$ is incident to two outer edges. The edges with the colours $j$ and $k$ are of the type $b^{\prime}$, and therefore $w_{2}^{m}=w_{3}^{m}=b^{\prime}$ (there are no words with $b^{\prime}$ in the set $W_{v}$, and thus $\left.w_{3}^{m}=b^{\prime}\right)$. But then $w^{n}$ and $w^{m}$ cannot be adjacent because $w^{m}$ cannot 

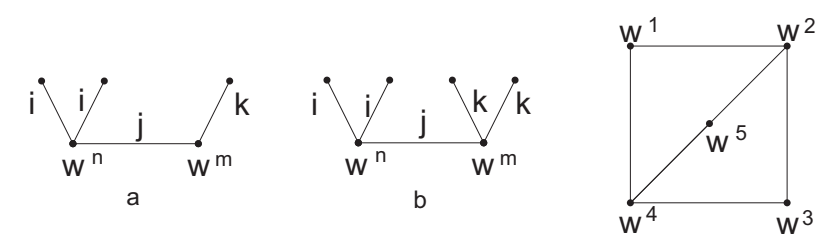

Figure 13: Forbidden colourings ( $a$ and $b)$.

contain more than two letters $b^{\prime}$. A contradiction. The proof that the colouring in Figure $13 b$ is forbidden is similar.

Observe now that the outer edges can be coloured with at most five colours from the set $\{1, \ldots, 6\}$ because if two or more outer edges are incident to some $w \in W \backslash W_{v}$, then they have to be of the same colour. This follows from the fact that every $w \in W \backslash W_{v}$ which is adjacent to at least two vertices from $W_{v}$ contains exactly one letter $b^{\prime}$. On the other hand, if the two outer edges $(w, u)$ and $(w, q)$, where $u, q \in W_{v}$, have the colours $i, j \in[d], i \neq j$, then $w_{i}=w_{j}=b^{\prime}$ because $u$ and $q$ have no letter $b^{\prime}$. A contradiction.

Assume that the outer edges are coloured with the colours $\{1,2,3,4,5\}$. Thus, two inner edges have to be coloured by the colour 6 . Since the colourings in Figures $13 a, b$ are forbidden, each endpoints of an inner edge with the colour 6 can be incident to at most one outer edge. Therefore, the inner edges are coloured by at least four colours: Two inner edges are coloured by the colour 6 and three inner edges are coloured by three colours $i, j, k \in\{1, \ldots, 5\}$. Thus, there are at least five inner edges. By Lemma 8, the graph $G$ cannot contain an odd cycles. Therefore, the inner edges coincide with at least five edges in the graph which is presented in Figure 13 on the right. Since the inner edges are coloured with at least four colours (two edges with the colour 6 and three edges with the colours $i, j, k$ ) there is a cycle in $G$ of length four containing three edges coloured by three colours. This is, by Lemma 8, impossible.

The situation does not improve (is even worse) if the outer edges are coloured with less than five colours or if $\left|W_{v}\right| \geqslant 8$. In the same way we prove the lemma in the cases $d=4,5$.

Let $S=\left\{a, a^{\prime}, b, b^{\prime}\right\}, d \in\{4,5,6\}$, and let $V, W \subset S^{d}$ be disjoint sets which are equivalent polybox codes without twin pairs. By (2.4), there are $v, u \in V$ such that $v_{i} \in\left\{u_{i}, u_{i}^{\prime}\right\}$ for every $i \in[d]$ and $\left|\left\{i \in[d]: v_{i}=u_{i}^{\prime}\right\}\right|=3$ for $d=4$ and $\mid\left\{i \in[d]: v_{i}=\right.$ $\left.u_{i}^{\prime}\right\} \mid \in\{3,5\}$ for $d=5,6$. From now on, without loss of generality, we assume that

$$
\begin{gathered}
v=b b b b, u=b^{\prime} b^{\prime} b^{\prime} b \quad \text { for } \quad d=4, \\
v=b b b b b, u=b^{\prime} b^{\prime} b^{\prime} b b \quad \text { or } \quad v=b b b b b, u=b^{\prime} b^{\prime} b^{\prime} b^{\prime} b^{\prime} \quad \text { for } \quad d=5 \\
v=b b b b b b, u=b^{\prime} b^{\prime} b^{\prime} b b b \quad \text { or } \quad v=b b b b b b, u=b^{\prime} b^{\prime} b^{\prime} b^{\prime} b^{\prime} b \quad \text { for } \quad d=6 .
\end{gathered}
$$

Since $V$ and $W$ are equivalent, by (2.6), we have $\breve{v} \cup \breve{u} \subset \cup E(W)$.

Let $R$ be as in Corollary 20, and let

$$
W_{v, u}=\{w \in W: \breve{w} \cap(\breve{v} \cup \breve{u}) \neq \emptyset\} \quad \text { and } \quad\left(\left|W_{v, u}^{i, l}\right|,\left|W_{v, u}^{i, l^{\prime}}\right|\right) \notin R \cup\{(12,0)\}
$$


for every $i \in[d]$ and $l \in S$. Note that the first equality means, again by (2.6), that $v, u \sqsubseteq W_{v, u}$.

Our further proceedings is described within the following three points:

1. For every $d \in\{4,5,6\}$ we first compute all twin pair free codes $W_{v, u} \subset S^{d}$ with $\left|W_{v, u}\right| \leqslant 12$ satisfying (5.1).

2. Next, we append, if necessary, the missing words $M=M\left(W_{v, u}\right) \subset S^{d}$ to the code $W_{v, u}$ such that the set of words $W_{v, u} \cup M$ is a polybox codes without twin pair, $\left|W_{v, u} \cup M\right|=12$ and $\left|\left(W_{v, u} \cup M\right)^{i, l}\right| \neq 12$ for every $i \in[d]$ and $l \in S$.

A comment: Observe that, if $\left(\left|W_{v, u}^{i, l}\right|,\left|W_{v, u}^{i, l^{\prime}}\right|\right) \in R$ for some $i \in[d]$ and some $l \in S$, then $\left(\left|\left(W_{v, u} \cup M\right)^{i, l}\right|,\left|\left(W_{v, u} \cup M\right)^{i, l^{\prime}}\right|\right) \in R \cup\{(12,0)\}$. If now $V$ is a twin pair free polybox code which is equivalent to $W_{v, u} \cup M$, disjoint with it and $\left(\left|\left(W_{v, u} \cup M\right)^{i, l}\right|,\left|\left(W_{v, u} \cup M\right)^{i, l^{\prime}}\right|\right) \neq$ $(12,0)$, then, by Corollary 20, $\left|W_{v, u} \cup M\right|>12$, a contradiction. That is why we may consider only the codes $W_{v, u}$ whose distributions of the words satisfy (5.1). As we see the codes $W_{v, u} \cup M$ such that $\left|\left(W_{v, u} \cup M\right)^{i, l}\right|=12$ for some $i \in[d]$ and some $l \in S$ will not considered during the computations.

3. Finally, we select only these codes $W_{v, u} \cup M$ which, by Lemma 19, contain all siblings: For every $i \in[d]$ and every $\{l, s\} \in\left\{\{a, b\},\left\{a, b^{\prime}\right\},\left\{a^{\prime}, b\right\},\left\{a^{\prime}, b^{\prime}\right\}\right\}$ there are two words $p, q \in W_{v, u} \cup M$ which are $i$-siblings such that $\left\{p_{i}, q_{i}\right\}=\{l, s\}$. (Every code of the form $W_{v, u} \cup M$ will our candidate for the code $W$ defined at the beginning of Section 5 . The code $V$ will be find in the proof of Theorem 27.)

Let

$$
W_{v}=\{w \in W: \breve{v} \cap \breve{w} \neq \emptyset\} \quad \text { and } \quad W_{u}=\{w \in W: \breve{u} \cap \breve{w} \neq \emptyset\} .
$$

Then, $v \sqsubseteq W_{v}$ and $u \sqsubseteq W_{u}$. Since $W_{v, u}=W_{v} \cup\left(W_{u} \backslash W_{v}\right)$ for some $W_{v}$ and $W_{u}$, to determine all covers $W_{v, u}$ with $\left|W_{v, u}\right| \leqslant 12$ we first compute all covers $W_{v}$ and $W_{u}$ with the number of words described in Lemma 22: $\left|W_{v}\right|,\left|W_{u}\right| \leqslant 8$ for $d=4,\left|W_{v}\right|,\left|W_{u}\right| \leqslant 7$ for $d=5$ and $\left|W_{v}\right|,\left|W_{u}\right| \leqslant 6$ for $d=6$. Obviously, in all cases, by Lemma $9,\left|W_{v}\right|,\left|W_{u}\right| \geqslant 5$.

By $\operatorname{Cov}_{v}$ and $\operatorname{Cov}_{u}$ we denote the family of all covers $W_{v}$ and $W_{u}$, respectively with the above described number of words.

By $\operatorname{Cov}_{v, u}$ we denote the family of all covers $W_{v, u}$ with $\left|W_{v, u}\right| \leqslant 12$ satisfying (5.1).

The family $\mathrm{Cov}_{v, u}$ can be easily computed from $\mathrm{Cov}_{v}$ and $\mathrm{Cov}_{u}$ as follows: For every $W_{v} \in \operatorname{Cov}_{v}$ and $W_{u} \in \operatorname{Cov}_{u}$ if the code $W_{v, u}=W_{v} \cup\left(W_{u} \backslash W_{v}\right)$ consists of at most twelve pairwise dichotomous words, does not contain a twin pair and its distribution of words satisfies (5.1), then $W_{v, u}$ is added to $\operatorname{Cov}_{v, u}$.

For every $d \in\{4,5,6\}$ we give non-isomorphic codes in $\operatorname{Cov}_{v}$ and $\operatorname{Cov}_{u}$ but to compute $\operatorname{Cov}_{v, u}$ we use all codes in $\operatorname{Cov}_{v}$ and $\operatorname{Cov}_{u}$. Having computed the family $\operatorname{Cov}_{v, u}$ we will find non-isomorphic codes $W_{v, u}$ in the family $\operatorname{Cov}_{v, u}$, and to these non-isomorphic codes we will try attach the missing words $M$ in the fashion described previously. Obviously, if the code $W_{v, u} \cup M$ contains all siblings and has twelve words without twin pairs, and $h \circ \sigma^{*}\left(W_{v, u}\right) \in \operatorname{Cov}_{v, u}$ is isomorphic to $W_{v, w}$, then the code $h \circ \sigma^{*}\left(W_{v, u} \cup M\right)$ also contains all siblings and does not contain a twin pair.

We now describe in details the way of computing the codes $W_{v, u} \cup M$ for $d=4$. 


\section{Case $d=4$}

By (2.5) and (2.6), for every $w \in W_{b b b b}$ we have

$$
g(w, b b b b)=2^{i} \text { for } i \in\{0, \ldots, 3\}
$$

and

$$
\sum_{w \in W_{b b b b}} g(w, b b b b)=16 .
$$

Let $x_{i}$ denote the number of words in $W_{b b b b}$ with $i$ letters $b$ (compare the last part of Section 2.8). Thus, admissible values of $x_{i}$ for $i=0,1,2,3$ are the solutions of the systems of the equations

$$
x_{0}+2 x_{1}+4 x_{2}+8 x_{3}=16, \quad x_{0}+x_{1}+x_{2}+x_{3}=m
$$

where $x_{0}, x_{1}, x_{2}, x_{3}$ are non-negative integers and $m=\left|W_{b b b b}\right|$. Below we list the positive solutions of (5.2) for $m=7,8$ (for $m=5,6$ the form of the cover $W_{b b b b}$ is described in Lemma 9 and 10):

For $m=7$ we have

$$
\begin{gathered}
x_{1}=6, x_{2}=1 \quad \text { or } \quad x_{0}=2, x_{1}=3, x_{2}=2 \quad \text { or } \\
x_{0}=4, x_{2}=3 \quad \text { or } \quad x_{0}=4, x_{1}=2, x_{3}=1 .
\end{gathered}
$$

For $m=8$ we have

$$
\begin{gathered}
x_{1}=8 \text { or } x_{0}=2, x_{1}=5, x_{2}=1 \quad \text { or } \\
x_{0}=4, x_{1}=2, x_{2}=2 \text { or } x_{0}=6, x_{1}=1, x_{3}=1 .
\end{gathered}
$$

Every solution of (5.2) gives a necessary distribution of words in a cover $W_{b b b b}$ with respect to a number of the letters $b$ in every word. For example, if $\left|W_{b b b b}\right|=8$ and $W_{b b b b}(i b)$ denotes the set of all words in $W_{b b b b}$ having $i$ letters $b$, then the distribution $\left(\left|W_{b b b b}(0 b)\right|,\left|W_{b b b b}(1 b)\right|,\left|W_{b b b b}(2 b)\right|,\left|W_{b b b b}(3 b)\right|\right)$ can take only one of the forms given by the solutions of $(5.2)$ for $m=8$ : $(0,8,0,0),(2,5,1,0),(4,2,2,0)$ or $(6,1,0,1)$. By $\operatorname{Cov}_{b b b b}\left(i_{1}, i_{2}, i_{3}, i_{4}\right)$ we denote the family of all covers $W_{b b b b}$ such that

$$
\left(\left|W_{b b b b}(0 b)\right|,\left|W_{b b b b}(1 b)\right|,\left|W_{b b b b}(2 b)\right|,\left|W_{b b b b}(3 b)\right|\right)=\left(i_{1}, i_{2}, i_{3}, i_{4}\right) .
$$

In Table 2 we present the results of the computations for $d=4$ (we present only non-empty sets $\left.\operatorname{Cov}_{b b b b}\left(i_{1}, i_{2}, i_{3}, i_{4}\right)\right)$ :

\begin{tabular}{|l|l|}
\hline Total & Non-isomorphic codes \\
\hline$\left|\operatorname{Cov}_{b b b b}(0,2,3,0)\right|=32$ & $a a a b, a^{\prime} a^{\prime} a^{\prime} b, b a a^{\prime} b, a^{\prime} b a b, a a^{\prime} b b$ \\
\hline$\left|\operatorname{Cov}_{b b b b}(2,3,0,1)\right|=64$ & $a a a a, a^{\prime} a^{\prime} a^{\prime} a, b a a^{\prime} a, a^{\prime} b a a, a a^{\prime} b a, b b b a^{\prime}$ \\
\hline & $a a a a, a a a^{\prime} b, a a^{\prime} a^{\prime} a^{\prime}, a^{\prime} a b a, a^{\prime} b a^{\prime} a^{\prime}, b a^{\prime} b a, b b a a^{\prime} ;$ \\
$\left|\operatorname{Cov}_{b b b b}(2,3,2,0)\right|=576$ & $a a a b, a a^{\prime} b a, a^{\prime} b a a^{\prime}, a a a^{\prime} a, a a^{\prime} a a^{\prime}, a^{\prime} b b a, b b a^{\prime} a^{\prime}$ \\
\hline$\left|\operatorname{Cov}_{b b b b}(2,5,1,0)\right|=192$ & $a a a b, a b a^{\prime} a^{\prime}, a^{\prime} a a a, a^{\prime} a b a^{\prime}, a^{\prime} a^{\prime} a^{\prime} a^{\prime}, b a a^{\prime} a, b a^{\prime} a a^{\prime}, b a^{\prime} b a$ \\
\hline$\left|\operatorname{Cov}_{b b b b}(0,8,0,0)\right|=8$ & $a a a b, a a^{\prime} b a^{\prime}, a b a^{\prime} a, a^{\prime} a b a, a^{\prime} a^{\prime} a^{\prime} b, a^{\prime} b a a^{\prime}, b a a^{\prime} a^{\prime}, b a^{\prime} a a$ \\
\hline
\end{tabular}


Table 2 .

If we now make the substitution $b \rightarrow b^{\prime}$ at the first three positions in every word in the covers from Table 2, we obtain $\operatorname{Cov}_{b^{\prime} b^{\prime} b^{\prime} b}\left(i_{1}, i_{2}, i_{3}, i_{4}\right)$ for every $\left(i_{1}, i_{2}, i_{3}, i_{4}\right)$ from the set $\{(0,2,3,0),(2,3,0,1),(2,3,2,0),(2,5,1,0),(0,8,0,0)\}$. Let $v=b b b b$ and $u=b^{\prime} b^{\prime} b^{\prime} b$, and let

$\operatorname{Cov}_{v}=\operatorname{Cov}_{v}(0,2,3,0) \cup \operatorname{Cov}_{v}(2,3,0,1) \cup \operatorname{Cov}_{v}(2,3,2,0) \cup \operatorname{Cov}_{v}(2,5,1,0) \cup \operatorname{Cov}_{v}(0,8,0,0)$, $\operatorname{Cov}_{u}=\operatorname{Cov}_{u}(0,2,3,0) \cup \operatorname{Cov}_{u}(2,3,0,1) \cup \operatorname{Cov}_{u}(2,3,2,0) \cup \operatorname{Cov}_{u}(2,5,1,0) \cup \operatorname{Cov}_{u}(0,8,0,0)$.

The simple computations show that $\left|\mathrm{Cov}_{v, u}\right|=312$ from which five codes are nonisomorphic:

$$
\begin{gathered}
W_{v, u}^{0}=\left\{a^{\prime} a^{\prime} a^{\prime} b, a^{\prime} b a a^{\prime}, b a a^{\prime} a, a a^{\prime} a^{\prime} a, a^{\prime} a a^{\prime} a^{\prime}, a b b a^{\prime}, b b a a, a b^{\prime} a^{\prime} a^{\prime}, b^{\prime} a b^{\prime} a, b^{\prime} a^{\prime} a a, b^{\prime} b^{\prime} a a^{\prime}\right\} \\
W_{v, u}^{1}=\left\{a a a a, a a a^{\prime} b, a a^{\prime} a^{\prime} a^{\prime}, a a^{\prime} b a, a b a a^{\prime}, a^{\prime} a b^{\prime} a, a^{\prime} b b b, a^{\prime} b^{\prime} a^{\prime} a^{\prime}, b^{\prime} a^{\prime} b^{\prime} a, b^{\prime} b^{\prime} a a^{\prime}\right\} \\
W_{v, u}^{2}=\left\{a a b a, a a b^{\prime} a^{\prime}, a a^{\prime} b b, a b^{\prime} b^{\prime} a, a^{\prime} a b^{\prime} b, a^{\prime} a^{\prime} b a^{\prime}, a^{\prime} a^{\prime} b^{\prime} a, a^{\prime} b b a, b a b a^{\prime}, b^{\prime} a^{\prime} b^{\prime} a^{\prime}\right\} \\
W_{v, u}^{3}=\left\{a a b a, a a b^{\prime} b, a a^{\prime} b b, a a^{\prime} b^{\prime} a^{\prime}, a^{\prime} a b^{\prime} a, a^{\prime} a^{\prime} b a^{\prime}, a^{\prime} b b a, a^{\prime} b^{\prime} b^{\prime} a^{\prime}, b a b a^{\prime}, b^{\prime} a^{\prime} b^{\prime} a\right\} \\
W_{v, u}^{4}=\left\{a a b a, a a b^{\prime} b, a a^{\prime} b b, a a^{\prime} b^{\prime} a, a^{\prime} a b^{\prime} a^{\prime}, a^{\prime} a^{\prime} b a^{\prime}, a^{\prime} b b a, a^{\prime} b^{\prime} b^{\prime} a, b a b a^{\prime}, b^{\prime} a^{\prime} b^{\prime} a^{\prime}\right\} .
\end{gathered}
$$

Finally, to these five codes we append the missing words $M^{j}, j \in\{0, \ldots, 4\}$, according the rules we gave before: The code $W_{v, u}^{0}$ has to be extended by one word, and the rest four codes by two words each. In all resulting polybox codes without twin pairs we check whether they posses all siblings (see the point 3 below (5.1)). The following two claims summarize our computations in dimension four.

Claim 23. The word $b b^{\prime} a b^{\prime}$ is the sole for which the set $W=W_{v, u}^{0} \cup M^{0}$, where $M^{0}=$ $\left\{b b^{\prime} a b^{\prime}\right\}$, is a polybox code without twin pair containing all siblings.

Claim 24. For every pair $t, r \in S^{4}$ of dichotomous words and every $j \in[4]$ if the set $W_{v, u}^{j} \cup\{t, r\}$ is a polybox code without twin pairs, then it does not contain all siblings: There is $i \in[4]$ and a pair of letters $\{l, s\} \in\left\{\{a, b\},\left\{a, b^{\prime}\right\},\left\{a^{\prime}, b\right\},\left\{a^{\prime}, b^{\prime}\right\}\right\}$ such that there are no two words $p, w \in W_{v, u}^{j} \cup\{t, r\}$ which are $i$-siblings and $\left\{p_{i}, w_{i}\right\}=\{l, s\}$. Thus, by Lemma 19, for every $j \in[4]$ the code $W_{v, u}^{j}$ cannot be extended to a polybox code $W_{v, u}^{j} \cup M^{j}$ with twelve words without twin pairs such that there is a code $V$ without twin pairs which is disjoint with $W_{v, u}^{j} \cup M^{j}$ and equivalent to it.

\section{Case $d=5$}

For $d=5$, by Lemmas 9, 10 and 22 we consider only one system of the equations:

$$
x_{0}+2 x_{1}+4 x_{2}+8 x_{3}+16 x_{4}=32, \quad x_{0}+x_{1}+x_{2}+x_{3}+x_{4}=7
$$

where $x_{0}, x_{1}, x_{2}, x_{3}, x_{4}$ are non-negative integers. It has the following positive solutions: 


$$
\begin{gathered}
x_{2}=6, x_{3}=1 \quad \text { or } x_{1}=2, x_{2}=5, x_{3}=1 \quad \text { or } \quad x_{1}=2, x_{2}=3, x_{3}=2 \\
\text { or } \quad x_{0}=2, x_{1}=1, x_{2}=1, x_{3}=3 \quad \text { or } \quad x_{0}=2, x_{1}=1, x_{2}=1, x_{3}=3 \\
\text { or } \quad x_{0}=2, x_{1}=1, x_{2}=3, x_{4}=1 \quad \text { or } \quad x_{0}=2, x_{1}=3, x_{3}=1, x_{4}=1 \\
\quad \text { or } \quad x_{1}=4, x_{2}=2, x_{4}=1 \quad \text { or } \quad x_{0}=4, x_{2}=1, x_{3}=1, x_{4}=1
\end{gathered}
$$

The results of the computations for $d=5$ are presented in Table 3 .

\begin{tabular}{|l|l|}
\hline Total & Non-isomorphic codes \\
\hline$\left|\operatorname{Cov}_{b b b b b}(0,0,2,3,0)\right|=80$ & $a a a b b, a^{\prime} a^{\prime} a^{\prime} b b, b a a^{\prime} b b, a^{\prime} b a b b, a a^{\prime} b b b$ \\
\hline$\left|\operatorname{Cov}_{b b b b b}(0,2,3,0,1)\right|=320$ & $a a a b a, a^{\prime} a^{\prime} a^{\prime} b a, b a a^{\prime} b a, a^{\prime} b a b a, a a^{\prime} b b a, b b b b a^{\prime}$ \\
\hline$\left|\operatorname{Cov}_{b b b b b}(2,3,1,0,1)\right|=640$ & $a a a a a, a^{\prime} a^{\prime} a^{\prime} a a, b a a^{\prime} a a, a^{\prime} b a a a, a a^{\prime} b a a, b b b a a^{\prime}, b b b a^{\prime} b$ \\
\hline$\left|\operatorname{Cov}_{b b b b b}(0,2,3,2,0)\right|=2880$ & $\begin{array}{l}a a a a b, a a a^{\prime} b b, a a^{\prime} a^{\prime} a^{\prime} b, a^{\prime} a b a b, a^{\prime} b a^{\prime} a^{\prime} b, b a^{\prime} b a b, b b a a^{\prime} b ; \\
a a a b b, a a^{\prime} b a b, a^{\prime} b a a^{\prime} b, a a a^{\prime} a b, a a^{\prime} a a^{\prime} b, a^{\prime} b b a b, b b a^{\prime} a^{\prime} b\end{array}$ \\
\hline
\end{tabular}

Table 3 .

For the pair $v=b b b b b, u=b^{\prime} b^{\prime} b^{\prime} b b$ we obtained $\left|\operatorname{Cov}_{v, u}\right|=2400$ from which fifteen

\begin{tabular}{|c|c|}
\hline No. & Non-isomorphic codes in $\operatorname{Cov}_{v, u}$ for $v=b b b b b, u=b^{\prime} b^{\prime} b^{\prime} b b$ \\
\hline 1. & $a a b a b, a a b^{\prime} b a, a a^{\prime} b b b, a b^{\prime} b^{\prime} b a^{\prime}, a^{\prime} a b^{\prime} b b, a^{\prime} a^{\prime} b a^{\prime} b, a^{\prime} a^{\prime} b^{\prime} b a^{\prime}, a^{\prime} b b a b, b a b a^{\prime} b, b^{\prime} a^{\prime} b^{\prime} b a$ \\
\hline 2. & $a a b a b, a a b^{\prime} b b, a a^{\prime} b b b, a a^{\prime} b^{\prime} b a, a^{\prime} a b^{\prime} b a^{\prime}, a^{\prime} a^{\prime} b a^{\prime} b, a^{\prime} b b a b, a^{\prime} b^{\prime} b^{\prime} b a, b a b a^{\prime} b, b^{\prime} a^{\prime} b^{\prime} b a^{\prime}$ \\
\hline 3. & $a a b a b, a a^{\prime} b b b, a b^{\prime} b^{\prime} a a, a b^{\prime} b^{\prime} a^{\prime} b, a^{\prime} a^{\prime} b a^{\prime} b, a^{\prime} b b a b, a^{\prime} b^{\prime} b^{\prime} a^{\prime} a^{\prime}, a^{\prime} b^{\prime} b^{\prime} b a, b a b a^{\prime} b, b^{\prime} b^{\prime} b^{\prime} a a^{\prime}$ \\
\hline 4. & $a a b a b, a a^{\prime} b b b, a b^{\prime} b^{\prime} a a, a b^{\prime} b^{\prime} b a^{\prime}, a^{\prime} a^{\prime} b a^{\prime} b, a^{\prime} b b a b, a^{\prime} b^{\prime} b^{\prime} a b, a^{\prime} b^{\prime} b^{\prime} a^{\prime} a^{\prime}, b a b a^{\prime} b, b^{\prime} b^{\prime} b^{\prime} a^{\prime} a$ \\
\hline 5. & $a a b a b, a a^{\prime} b b b, a b^{\prime} b^{\prime} a b, a b^{\prime} b^{\prime} a^{\prime} a, a^{\prime} a^{\prime} b a^{\prime} b, a^{\prime} b b a b, a^{\prime} b^{\prime} b^{\prime} a a^{\prime}, a^{\prime} b^{\prime} b^{\prime} b a, b a b a^{\prime} b, b^{\prime} b^{\prime} b^{\prime} a^{\prime} a^{\prime}$ \\
\hline 6. & $a a b a b, a a^{\prime} b b b, a b^{\prime} b^{\prime} a^{\prime} a, a b^{\prime} b^{\prime} b a^{\prime}, a^{\prime} a^{\prime} b a^{\prime} b, a^{\prime} b b a b, a^{\prime} b^{\prime} b^{\prime} a a^{\prime}, a^{\prime} b^{\prime} b^{\prime} a^{\prime} b, b a b a^{\prime} b, b^{\prime} b^{\prime} b^{\prime} a a$ \\
\hline 7. & $a b b a a, a b b a^{\prime} b, a b^{\prime} b^{\prime} a a, a b^{\prime} b^{\prime} a^{\prime} b, a^{\prime} b b a^{\prime} a^{\prime}, a^{\prime} b b b a, a^{\prime} b^{\prime} b^{\prime} a^{\prime} a^{\prime}, a^{\prime} b^{\prime} b^{\prime} b a, b b b a a^{\prime}, b^{\prime} b^{\prime} b^{\prime} a a^{\prime}$ \\
\hline 8. & $a b b a a, a b b a^{\prime} b, a b^{\prime} b^{\prime} a a, a b^{\prime} b^{\prime} b a^{\prime}, a^{\prime} b b a^{\prime} a^{\prime}, a^{\prime} b b b a, a^{\prime} b^{\prime} b^{\prime} a b, a^{\prime} b^{\prime} b^{\prime} a^{\prime} a^{\prime}, b b b a a^{\prime}, b^{\prime} b^{\prime} b^{\prime} a^{\prime} a$ \\
\hline 9. & $a b b a a, a b b a^{\prime} b, a b^{\prime} b^{\prime} a a^{\prime}, a b^{\prime} b^{\prime} a^{\prime} b, a^{\prime} b b a^{\prime} a^{\prime}, a^{\prime} b b b a, a^{\prime} b^{\prime} b^{\prime} a^{\prime} a, a^{\prime} b^{\prime} b^{\prime} b a^{\prime}, b b b a a^{\prime}, b^{\prime} b^{\prime} b^{\prime} a a$ \\
\hline 10. & $a b b a a, a b b a^{\prime} b, a b^{\prime} b^{\prime} a a^{\prime}, a b^{\prime} b^{\prime} b a, a^{\prime} b b a^{\prime} a^{\prime}, a^{\prime} b b b a, a^{\prime} b^{\prime} b^{\prime} a b, a^{\prime} b^{\prime} b^{\prime} a^{\prime} a, b b b a a^{\prime}, b^{\prime} b^{\prime} b^{\prime} a^{\prime} a^{\prime}$ \\
\hline 11. & $a b b a a, a b b a^{\prime} b, a b^{\prime} b^{\prime} a b, a b^{\prime} b^{\prime} a^{\prime} a^{\prime}, a^{\prime} b b a^{\prime} a^{\prime}, a^{\prime} b b b a, a^{\prime} b^{\prime} b^{\prime} a a, a^{\prime} b^{\prime} b^{\prime} b a^{\prime}, b b b a a^{\prime}, b^{\prime} b^{\prime} b^{\prime} a^{\prime} a$ \\
\hline 12. & $a b b a a, a b b a^{\prime} b, a b^{\prime} b^{\prime} a^{\prime} a^{\prime}, a b^{\prime} b^{\prime} b a, a^{\prime} b b a^{\prime} a^{\prime}, a^{\prime} b b b a, a^{\prime} b^{\prime} b^{\prime} a a, a^{\prime} b^{\prime} b^{\prime} a^{\prime} b, b b b a a^{\prime}, b^{\prime} b^{\prime} b^{\prime} a a^{\prime}$ \\
\hline 13. & $a b b a a, a b b a^{\prime} b, a^{\prime} b b a^{\prime} a^{\prime}, a^{\prime} b b b a, b b b a a^{\prime}, b^{\prime} a b^{\prime} a a, b^{\prime} a b^{\prime} a^{\prime} b, b^{\prime} a^{\prime} b^{\prime} a^{\prime} a^{\prime}, b^{\prime} a^{\prime} b^{\prime} b a, b^{\prime} b^{\prime} b^{\prime} a a^{\prime}$ \\
\hline 14. & $a b b a a, a b b a^{\prime} b, a^{\prime} b b a^{\prime} a^{\prime}, a^{\prime} b b b a, b b b a a^{\prime}, b^{\prime} a b^{\prime} a a, b^{\prime} a b^{\prime} b a^{\prime}, b^{\prime} a^{\prime} b^{\prime} a b, b^{\prime} a^{\prime} b^{\prime} a^{\prime} a^{\prime}, b^{\prime} b^{\prime} b^{\prime} a^{\prime} a$ \\
\hline 15. & $a b b a a, a b b a^{\prime} b, a^{\prime} b b a^{\prime} a^{\prime}, a^{\prime} b b b a, b b b a a^{\prime}, b^{\prime} a b^{\prime} a a^{\prime}, b^{\prime} a b^{\prime} a^{\prime} b, b^{\prime} a^{\prime} b^{\prime} a^{\prime} a, b^{\prime} a^{\prime} b^{\prime} b a^{\prime}, b^{\prime} b^{\prime} b^{\prime} a a$ \\
\hline
\end{tabular}
codes are non-isomorphic (Table 4), and for the pair $v=b b b b b, q=b^{\prime} b^{\prime} b^{\prime} b^{\prime} b^{\prime}$ we obtained $\left|\operatorname{Cov}_{v, q}\right|=4480$ from which seven codes are non-isomorphic (Table 5). Every code $Q \in$ $\operatorname{Cov}_{v, u} \cup \operatorname{Cov}_{v, q}$ has ten words.

Table 4. 


\begin{tabular}{|l|l|}
\hline No. & Non-isomorphic codes in $\operatorname{Cov}_{v, q}$ for $v=b b b b b, q=b^{\prime} b^{\prime} b^{\prime} b^{\prime} b^{\prime}$ \\
\hline 1. & $a a a b b, a a a b^{\prime} b^{\prime}, a a^{\prime} b b b, a a^{\prime} b^{\prime} b^{\prime} b^{\prime}, a^{\prime} a^{\prime} a^{\prime} b b, a^{\prime} a^{\prime} a^{\prime} b^{\prime} b^{\prime}, a^{\prime} b a b b, a^{\prime} b^{\prime} a b^{\prime} b^{\prime}, b a a^{\prime} b b, b^{\prime} a a^{\prime} b^{\prime} b^{\prime}$ \\
\hline 2. & $a a a b b, a a a b^{\prime} b^{\prime}, a a^{\prime} b b b, a b^{\prime} a^{\prime} b^{\prime} b^{\prime}, a^{\prime} a b^{\prime} b^{\prime} b^{\prime}, a^{\prime} a^{\prime} a^{\prime} b b, a^{\prime} a^{\prime} a^{\prime} b^{\prime} b^{\prime}, a^{\prime} b a b b, b a a^{\prime} b b, b^{\prime} a^{\prime} a b^{\prime} b^{\prime}$ \\
\hline 3. & $a a a b b, a a a^{\prime} b^{\prime} b^{\prime}, a a^{\prime} b b b, a a^{\prime} b^{\prime} b^{\prime} b^{\prime}, a^{\prime} a^{\prime} a b^{\prime} b^{\prime}, a^{\prime} a^{\prime} a^{\prime} b b, a^{\prime} b a b b, a^{\prime} b^{\prime} a^{\prime} b^{\prime} b^{\prime}, b a a^{\prime} b b, b^{\prime} a a b^{\prime} b^{\prime}$ \\
\hline 4. & $a a a b b, a a a^{\prime} b^{\prime} b^{\prime}, a a^{\prime} b b b, a b^{\prime} a b^{\prime} b^{\prime}, a^{\prime} a b^{\prime} b^{\prime} b^{\prime}, a^{\prime} a^{\prime} a b^{\prime} b^{\prime}, a^{\prime} a^{\prime} a^{\prime} b b, a^{\prime} b a b b, b a a^{\prime} b b, b^{\prime} a^{\prime} a^{\prime} b^{\prime} b^{\prime}$ \\
\hline 5. & $a a a b b, a a b^{\prime} a b^{\prime}, a a^{\prime} b b b, a a^{\prime} b^{\prime} b^{\prime} b^{\prime}, a^{\prime} a^{\prime} a^{\prime} b b, a^{\prime} a^{\prime} b^{\prime} a^{\prime} b^{\prime}, a^{\prime} b a b b, a^{\prime} b^{\prime} b^{\prime} a b^{\prime}, b a a^{\prime} b b, b^{\prime} a b^{\prime} a^{\prime} b^{\prime}$ \\
\hline 6. & $a a a b b, a a b^{\prime} a b^{\prime}, a a^{\prime} b b b, a b^{\prime} b^{\prime} a^{\prime} b^{\prime}, a^{\prime} a b^{\prime} b^{\prime} b^{\prime}, a^{\prime} a^{\prime} a^{\prime} b b, a^{\prime} a^{\prime} b^{\prime} a^{\prime} b^{\prime}, a^{\prime} b a b b, b a a^{\prime} b b, b^{\prime} a^{\prime} b^{\prime} a b^{\prime}$ \\
\hline 7. & $a a a b b, a a b^{\prime} b^{\prime} b^{\prime}, a a^{\prime} b b b, a a^{\prime} b^{\prime} a b^{\prime}, a^{\prime} a b^{\prime} a^{\prime} b^{\prime}, a^{\prime} a^{\prime} a^{\prime} b b, a^{\prime} b a b b, a^{\prime} b^{\prime} b^{\prime} a b^{\prime}, b a a^{\prime} b b, b^{\prime} a^{\prime} b^{\prime} a^{\prime} b^{\prime}$ \\
\hline
\end{tabular}

Table 5 .

Claim 25. Let $v=b b b b b, u=b^{\prime} b^{\prime} b^{\prime} b b$ and $q=b^{\prime} b^{\prime} b^{\prime} b^{\prime} b^{\prime}$. For every pair $t, r \in S^{5}$ of dichotomous words and every code $Q \in \operatorname{Cov}_{v, u} \cup \operatorname{Cov}_{v, q}$ if the set $Q \cup\{t, r\}$ is a polybox code without twin pairs, then it does not contain all siblings: There is $i \in[5]$ and a pair of letters $\{l, s\} \in\left\{\{a, b\},\left\{a, b^{\prime}\right\},\left\{a^{\prime}, b\right\},\left\{a^{\prime}, b^{\prime}\right\}\right\}$ such that there are no two words $p, w \in Q \cup\{t, r\}$ which are $i$-siblings and $\left\{p_{i}, w_{i}\right\}=\{l, s\}$. Thus, by Lemma 19, for every $Q \in \operatorname{Cov}_{v, u} \cup \operatorname{Cov}_{v, q}$ the code $Q$ cannot be extended to a polybox code $Q \cup M$ with twelve words without twin pairs such that there is a code $V$ without twin pairs which is disjoint with $Q \cup M$ and equivalent to it.

\section{Case $d=6$}

By Lemma 22, for $d=6$ we need all covers of the word bbbbbb by codes without twin pairs consisting of five and six words. By Lemmas 9 and 10, these codes have the forms (Table 6):

\begin{tabular}{|l|l|}
\hline Total & Non-isomorphic codes \\
\hline$\left|\operatorname{Cov}_{b b b b b b}(0,0,0,2,3,0)\right|=160$ & $a a a b b b, a^{\prime} a^{\prime} a^{\prime} b b b, b a a^{\prime} b b b, a^{\prime} b a b b b, a a^{\prime} b b b b$ \\
\hline$\left|\operatorname{Cov}_{b b b b b b}(0,0,2,3,0,1)\right|=960$ & $a a a b b a, a^{\prime} a^{\prime} a^{\prime} b b a, b a a^{\prime} b b a, a^{\prime} b a b b a, a a^{\prime} b b b a, b b b b b a^{\prime}$ \\
\hline
\end{tabular}

Table 6 .

For the pair $v=b b b b b b, u=b^{\prime} b^{\prime} b^{\prime} b b b$ we obtained $\left|\operatorname{Cov}_{v, u}\right|=8128$ from which fifteen codes are non-isomorphic (Table 7), and for the pair $v=b b b b b b, q=b^{\prime} b^{\prime} b^{\prime} b^{\prime} b^{\prime} b$ we obtained $\left|\operatorname{Cov}_{v, q}\right|=17918$ from which fifteen codes are non-isomorphic (Table 8). Every code $Q \in \operatorname{Cov}_{v, u} \cup \operatorname{Cov}_{v, q}$ has ten words. 


\begin{tabular}{|c|c|}
\hline No. & . \\
\hline 1. & $\begin{array}{l}a^{\prime} b^{\prime} b b b, a^{\prime} a^{\prime} b^{\prime} a^{\prime} b b, a^{\prime} b^{\prime} b^{\prime} a b b, b a b b a a^{\prime}, b a b b b a, b a^{\prime} b b a b, b a^{\prime} b b a^{\prime} a, \\
\prime a b^{\prime} a^{\prime} b b\end{array}$ \\
\hline 2. & $\begin{array}{l}b b b b, a^{\prime} a^{\prime} b a^{\prime} b b, a^{\prime} b b a b b, b a b a^{\prime} b b, b^{\prime} b^{\prime} b^{\prime} a a a, b^{\prime} b^{\prime} b^{\prime} a a^{\prime} b, b^{\prime} b^{\prime} b^{\prime} a^{\prime} a^{\prime} a^{\prime}, \\
{ }^{\prime} b^{\prime} b^{\prime} b a a^{\prime}\end{array}$ \\
\hline 3. & $\begin{array}{l}a^{\prime} b b, a b^{\prime} b^{\prime} a b a, a b^{\prime} b^{\prime} a^{\prime} b b, a^{\prime} b b a^{\prime} a^{\prime} b, a^{\prime} b b b a b, a^{\prime} b^{\prime} b^{\prime} a^{\prime} b a^{\prime}, a^{\prime} b^{\prime} b^{\prime} b b a, \\
b^{\prime} a b a^{\prime}\end{array}$ \\
\hline 4. & $\begin{array}{l}\text { bb, } a b^{\prime} b^{\prime} a b a, a b^{\prime} b^{\prime} b b a^{\prime}, a^{\prime} b b a^{\prime} a^{\prime} b, a^{\prime} b b b a b, a^{\prime} b^{\prime} b^{\prime} a b b, a^{\prime} b^{\prime} b^{\prime} a^{\prime} b a^{\prime}, \\
a^{\prime} b a\end{array}$ \\
\hline 5. & $\begin{array}{l}{ }^{\prime} b b, a b^{\prime} b^{\prime} a b b, a b^{\prime} b^{\prime} a^{\prime} b a, a^{\prime} b b a^{\prime} a^{\prime} b, a^{\prime} b b b a b, a^{\prime} b^{\prime} b^{\prime} a b a^{\prime}, a^{\prime} b^{\prime} b^{\prime} b b a \\
a^{\prime} b a^{\prime}\end{array}$ \\
\hline 6. & $\begin{array}{l}a^{\prime} b b, a b^{\prime} b^{\prime} a^{\prime} b a, a b^{\prime} b^{\prime} b b a^{\prime}, a^{\prime} b b a^{\prime} a^{\prime} b, a^{\prime} b b b a b, a^{\prime} b^{\prime} b^{\prime} a b a^{\prime}, a^{\prime} b^{\prime} b^{\prime} a^{\prime} b b, \\
b^{\prime} a b a\end{array}$ \\
\hline 7. & $\begin{array}{l}a^{\prime} b b, a^{\prime} b b a^{\prime} a^{\prime} b, a^{\prime} b b b a b, b b b a a^{\prime} b, b^{\prime} a b^{\prime} a b a, b^{\prime} a b^{\prime} a^{\prime} b b, b^{\prime} a^{\prime} b^{\prime} a^{\prime} b a^{\prime}, \\
b^{\prime} a b a^{\prime}\end{array}$ \\
\hline 8. & $\begin{array}{l}a^{\prime} b b, a^{\prime} b b a^{\prime} a^{\prime} b, a^{\prime} b b b a b, b b b a a^{\prime} b, b^{\prime} a b^{\prime} a b a, b^{\prime} a b^{\prime} b b a^{\prime}, b^{\prime} a^{\prime} b^{\prime} a b b, \\
b^{\prime} b^{\prime} a^{\prime} b a\end{array}$ \\
\hline 9 . & $\begin{array}{l}\prime b b, a^{\prime} b b a^{\prime} a^{\prime} b, a^{\prime} b b b a b, b b b a a^{\prime} b, b^{\prime} b^{\prime} b^{\prime} a a a, b^{\prime} b^{\prime} b^{\prime} a a^{\prime} b, b^{\prime} b^{\prime} b^{\prime} a^{\prime} a^{\prime} a^{\prime}, \\
b^{\prime} b a a^{\prime}\end{array}$ \\
\hline 10 . & $\begin{array}{l}{ }^{\prime} b b, a^{\prime} b b a^{\prime} a^{\prime} b, a^{\prime} b b b a b, b b b a a^{\prime} b, b^{\prime} b^{\prime} b^{\prime} a a c \\
b^{\prime} b^{\prime} b a^{\prime} a\end{array}$ \\
\hline 11. & $\begin{array}{l}b b, a^{\prime} b b a^{\prime} a^{\prime} b, a^{\prime} b b b a b, b b b a a^{\prime} b, b^{\prime} b^{\prime} b^{\prime} a a b, b^{\prime} b^{\prime} b^{\prime} a a^{\prime} a, b^{\prime} b^{\prime} b^{\prime} a^{\prime} a a^{\prime}, \\
b^{\prime} b a^{\prime} a^{\prime}\end{array}$ \\
\hline 12 . & $\begin{array}{l}a^{\prime} b, b b b a^{\prime} a^{\prime} a^{\prime}, b b b a^{\prime} b a, b b b b a a^{\prime}, b^{\prime} b^{\prime} b^{\prime} a a a, b^{\prime} b^{\prime} b^{\prime} a a^{\prime} b, b^{\prime} b^{\prime} b^{\prime} a^{\prime} a^{\prime} a^{\prime}, \\
\prime^{\prime} b a a^{\prime}\end{array}$ \\
\hline 13. & $\begin{array}{l}a a^{\prime} b, b b b a^{\prime} a^{\prime} a^{\prime}, b b b a^{\prime} b a, b b b b a a^{\prime}, b^{\prime} b^{\prime} b^{\prime} a a a, b^{\prime} b^{\prime} b^{\prime} a b a^{\prime}, b^{\prime} b^{\prime} b^{\prime} a^{\prime} a b \\
b^{\prime} b^{\prime} b^{\prime} b a^{\prime} a\end{array}$ \\
\hline 14. & $\begin{array}{l}b b b a a a, b b b a a^{\prime} b, b b b a^{\prime} a^{\prime} a^{\prime}, b b b a^{\prime} b a, b b b b a a^{\prime}, b^{\prime} b^{\prime} b^{\prime} a a a^{\prime}, b^{\prime} b^{\prime} b^{\prime} a a^{\prime} b, b^{\prime} b^{\prime} b^{\prime} a^{\prime} a^{\prime} a, \\
b^{\prime} b^{\prime} b^{\prime} a^{\prime} b a^{\prime}, b^{\prime} b^{\prime} b^{\prime} b a a\end{array}$ \\
\hline 15 & $b a a^{\prime} b, b b b a^{\prime} a^{\prime} a^{\prime}, b b b a^{\prime} b a, b b b b a a^{\prime}, b^{\prime} b^{\prime} b^{\prime} a a a^{\prime}, b^{\prime} b^{\prime} b^{\prime} a b a, b^{\prime} b^{\prime} b$ \\
\hline
\end{tabular}

Table 7 . 


\begin{tabular}{|c|c|}
\hline No. & on-isomorphic codes in $\mathrm{Cov}_{2}$ \\
\hline 1. & $\begin{array}{l}a a a b b b, a a b^{\prime} b^{\prime} b^{\prime} a, a a^{\prime} b b b b, a a^{\prime} b^{\prime} b^{\prime} b^{\prime} b, a^{\prime} a^{\prime} a^{\prime} b b b, a^{\prime} a^{\prime} b^{\prime} b^{\prime} b^{\prime} a^{\prime}, a^{\prime} b a b b b, a^{\prime} b^{\prime} b^{\prime} b^{\prime} b^{\prime} a \\
b a a^{\prime} b b b, b^{\prime} a b^{\prime} b^{\prime} b^{\prime} a^{\prime}\end{array}$ \\
\hline 2. & $\begin{array}{l}a a a b b b, a a b^{\prime} b^{\prime} b^{\prime} a, a a^{\prime} b b b b, a b^{\prime} b^{\prime} b^{\prime} b^{\prime} a^{\prime}, a^{\prime} a b^{\prime} b^{\prime} b^{\prime} b, a^{\prime} a^{\prime} a^{\prime} b b b, a^{\prime} a^{\prime} b^{\prime} b^{\prime} b^{\prime} a^{\prime}, a^{\prime} b a b b b \\
b a a^{\prime} b b b, b^{\prime} a^{\prime} b^{\prime} b^{\prime} b^{\prime} a\end{array}$ \\
\hline 3. & $\begin{array}{l}a a a b b b, a a b^{\prime} b^{\prime} b^{\prime} b, a a^{\prime} b b b b, a a^{\prime} b^{\prime} b^{\prime} b^{\prime} a, a^{\prime} a b^{\prime} b^{\prime} b^{\prime} a^{\prime}, a^{\prime} a^{\prime} a^{\prime} b b b, a^{\prime} b a b b b, a^{\prime} b^{\prime} b^{\prime} b^{\prime} b^{\prime} a \\
b a a^{\prime} b b b, b^{\prime} a^{\prime} b^{\prime} b^{\prime} b^{\prime} a^{\prime}\end{array}$ \\
\hline 4. & $\begin{array}{l}a a a b b b, a a^{\prime} b b b b, a b^{\prime} b^{\prime} a b^{\prime} a, a b^{\prime} b^{\prime} a^{\prime} b^{\prime} b, a^{\prime} a^{\prime} a^{\prime} b b b, a^{\prime} b a b b b, a^{\prime} b^{\prime} b^{\prime} a^{\prime} b^{\prime} a^{\prime}, a^{\prime} b^{\prime} b^{\prime} b^{\prime} b^{\prime} a \\
b a a^{\prime} b b b, b^{\prime} b^{\prime} b^{\prime} a b^{\prime} a^{\prime}\end{array}$ \\
\hline 5. & $\begin{array}{l}a a b b b a, a a b^{\prime} b^{\prime} b^{\prime} a, a a^{\prime} b b b b, a a^{\prime} b^{\prime} b^{\prime} b^{\prime} b, a^{\prime} a^{\prime} b b b a^{\prime}, a^{\prime} a^{\prime} b^{\prime} b^{\prime} b^{\prime} a^{\prime}, a^{\prime} b b b b a, a^{\prime} b^{\prime} b^{\prime} b^{\prime} b^{\prime} a \\
b a b b b a^{\prime}, b^{\prime} a b^{\prime} b^{\prime} b^{\prime} a^{\prime}\end{array}$ \\
\hline 6. & $\begin{array}{l}a a b b b a, a a b^{\prime} b^{\prime} b^{\prime} a, a a^{\prime} b b b b, a b^{\prime} b^{\prime} b^{\prime} b^{\prime} a^{\prime}, a^{\prime} a b^{\prime} b^{\prime} b^{\prime} b, a^{\prime} a^{\prime} b b b a^{\prime}, a^{\prime} a^{\prime} b^{\prime} b^{\prime} b^{\prime} a^{\prime}, a^{\prime} b b b b a \\
b a b b b a^{\prime}, b^{\prime} a^{\prime} b^{\prime} b^{\prime} b^{\prime} a\end{array}$ \\
\hline 7. & $\begin{array}{l}a a b b b a, a a b^{\prime} b^{\prime} b^{\prime} a^{\prime}, a a^{\prime} b b b b, a a^{\prime} b^{\prime} b^{\prime} b^{\prime} b, a^{\prime} a^{\prime} b b b a^{\prime}, a^{\prime} a^{\prime} b^{\prime} b^{\prime} b^{\prime} a, a^{\prime} b b b b a, a^{\prime} b^{\prime} b^{\prime} b^{\prime} b^{\prime} a^{\prime}, \\
b a b b b a^{\prime}, b^{\prime} a b^{\prime} b^{\prime} b^{\prime} a\end{array}$ \\
\hline 8. & $\begin{array}{l}a a b b b a, a a b^{\prime} b^{\prime} b^{\prime} a^{\prime}, a a^{\prime} b b b b, a b^{\prime} b^{\prime} b^{\prime} b^{\prime} a, a^{\prime} a b^{\prime} b^{\prime} b^{\prime} b, a^{\prime} a^{\prime} b b b a^{\prime}, a^{\prime} a^{\prime} b^{\prime} b^{\prime} b^{\prime} a, a^{\prime} b b b b a \\
b a b b b a^{\prime}, b^{\prime} a^{\prime} b^{\prime} b^{\prime} b^{\prime} a^{\prime}\end{array}$ \\
\hline 9. & $\begin{array}{l}a a b b b a, a a b^{\prime} b^{\prime} b^{\prime} b, a a^{\prime} b b b b, a a^{\prime} b^{\prime} b^{\prime} b^{\prime} a, a^{\prime} a b^{\prime} b^{\prime} b^{\prime} a^{\prime}, a^{\prime} a^{\prime} b b b a^{\prime}, a^{\prime} b b b b a, a^{\prime} b^{\prime} b^{\prime} b^{\prime} b^{\prime} a \\
b a b b b a^{\prime}, b^{\prime} a^{\prime} b^{\prime} b^{\prime} b^{\prime} a^{\prime}\end{array}$ \\
\hline 10. & $\begin{array}{l}a a b b b a, a a b^{\prime} b^{\prime} b^{\prime} b, a a^{\prime} b b b b, a a^{\prime} b^{\prime} b^{\prime} b^{\prime} a^{\prime}, a^{\prime} a b^{\prime} b^{\prime} b^{\prime} a, a^{\prime} a^{\prime} b b b a^{\prime}, a^{\prime} b b b b a, a^{\prime} b^{\prime} b^{\prime} b^{\prime} b^{\prime} a^{\prime} \\
b a b b b a^{\prime}, b^{\prime} a^{\prime} b^{\prime} b^{\prime} b^{\prime} a\end{array}$ \\
\hline 11. & $\begin{array}{l}a a b b b a, a a^{\prime} b b b b, a b^{\prime} a b^{\prime} b^{\prime} a, a b^{\prime} a^{\prime} b^{\prime} b^{\prime} b, a^{\prime} a^{\prime} b b b a^{\prime}, a^{\prime} b b b b a, a^{\prime} b^{\prime} a^{\prime} b^{\prime} b^{\prime} a^{\prime}, a^{\prime} b^{\prime} b^{\prime} b^{\prime} b^{\prime} a \\
b a b b b a^{\prime}, b^{\prime} b^{\prime} a b^{\prime} b^{\prime} a^{\prime}\end{array}$ \\
\hline 12. & $\begin{array}{l}a a b b b a, a a^{\prime} b b b b, a b^{\prime} a b^{\prime} b^{\prime} a, a b^{\prime} b^{\prime} b^{\prime} b^{\prime} a^{\prime}, a^{\prime} a^{\prime} b b b a^{\prime}, a^{\prime} b b b b a, a^{\prime} b^{\prime} a b^{\prime} b^{\prime} b, a^{\prime} b^{\prime} a^{\prime} b^{\prime} b^{\prime} a^{\prime} \\
b a b b b a^{\prime}, b^{\prime} b^{\prime} a^{\prime} b^{\prime} b^{\prime} a\end{array}$ \\
\hline 13. & $\begin{array}{l}a a b b b a, a a^{\prime} b b b b, a b^{\prime} a b^{\prime} b^{\prime} a^{\prime}, a b^{\prime} a^{\prime} b^{\prime} b^{\prime} b, a^{\prime} a^{\prime} b b b a^{\prime}, a^{\prime} b b b b a, a^{\prime} b^{\prime} a^{\prime} b^{\prime} b^{\prime} a, a^{\prime} b^{\prime} b^{\prime} b^{\prime} b^{\prime} a^{\prime}, \\
b a b b b a^{\prime}, b^{\prime} b^{\prime} a b^{\prime} b^{\prime} a\end{array}$ \\
\hline 14. & $\begin{array}{l}a a b b b a, a a^{\prime} b b b b, a b^{\prime} a b^{\prime} b^{\prime} a^{\prime}, a b^{\prime} b^{\prime} b^{\prime} b^{\prime} a, a^{\prime} a^{\prime} b b b a^{\prime}, a^{\prime} b b b b a, a^{\prime} b^{\prime} a b^{\prime} b^{\prime} b, a^{\prime} b^{\prime} a^{\prime} b^{\prime} b^{\prime} a \\
b a b b b a^{\prime}, b^{\prime} b^{\prime} a^{\prime} b^{\prime} b^{\prime} a^{\prime}\end{array}$ \\
\hline 15. & $\begin{array}{l}a a b b b a, a a^{\prime} b b b b, a^{\prime} a^{\prime} b b b a^{\prime}, a^{\prime} b b b b a, b a b b b a^{\prime}, b^{\prime} b^{\prime} a a b^{\prime} a, b^{\prime} b^{\prime} a a^{\prime} b^{\prime} b, b^{\prime} b^{\prime} a^{\prime} a^{\prime} b^{\prime} a^{\prime}, \\
b^{\prime} b^{\prime} a^{\prime} b^{\prime} b^{\prime} a, b^{\prime} b^{\prime} b^{\prime} a b^{\prime} a^{\prime}\end{array}$ \\
\hline
\end{tabular}

Table 8 .

For the same reason as for $d=5$ we have the following

Claim 26. Let $v=b b b b b b, u=b^{\prime} b^{\prime} b^{\prime} b b b$ and $q=b^{\prime} b^{\prime} b^{\prime} b^{\prime} b^{\prime} b$. For every $Q \in \operatorname{Cov}_{v, u} \cup \operatorname{Cov}_{v, q}$ the code $Q$ cannot be extended to a polybox code $Q \cup M$ with twelve words without twin pairs such that there is a code $V$ without twin pairs which is disjoint with $Q \cup M$ and equivalent to it.

We now can prove our key result on the form of two disjoint and equivalent polybox codes without twin pairs having twelve words each. 
Theorem 27. Let $d \in\{4,5,6\}$, and let $V, W \subset S^{d}$ be disjoint sets which are equivalent polybox codes without twin pairs. If $|V|=12$, then, up to an isomorphism,

$$
V_{A}=\left\{a a^{\prime} b b^{\prime}, a b b^{\prime} a, a b^{\prime} b^{\prime} b^{\prime}, a^{\prime} a b^{\prime} b^{\prime}, a^{\prime} a^{\prime} a b^{\prime}, a^{\prime} b b^{\prime} b, b a b b^{\prime}, b b b b, b b^{\prime} a^{\prime} b, b^{\prime} a b a^{\prime}, b^{\prime} a^{\prime} b b, b^{\prime} b^{\prime} b^{\prime} b\right\},
$$

$W_{A}=\left\{a^{\prime} a^{\prime} a^{\prime} b, a^{\prime} b a a^{\prime}, b a a^{\prime} a, a a^{\prime} a^{\prime} a, a^{\prime} a a^{\prime} a^{\prime}, a b b a^{\prime}, b b a a, a b^{\prime} a^{\prime} a^{\prime}, b^{\prime} a b^{\prime} a, b^{\prime} a^{\prime} a a, b^{\prime} b^{\prime} a a^{\prime}, b b^{\prime} a b^{\prime}\right\}$, where $A=\{1,2,3,4\} \subseteq[d]$ and $V_{A^{c}}=W_{A^{c}}=\left\{r_{A^{c}}\right\}$ for some $r \in S^{d}$.

Proof. Let $d=4$. We may assume, by (2.4), that $b b b b, b^{\prime} b^{\prime} b^{\prime} b \in V$. By Lemma 19, Claims 23 and 24, the code $W$ must be, up to an isomorphism, of the form

$$
W=\left\{a^{\prime} a^{\prime} a^{\prime} b, a^{\prime} b a a^{\prime}, b a a^{\prime} a, a a^{\prime} a^{\prime} a, a^{\prime} a a^{\prime} a^{\prime}, a b b a^{\prime}, b b a a, a b^{\prime} a^{\prime} a^{\prime}, b^{\prime} a b^{\prime} a, b^{\prime} a^{\prime} a a, b^{\prime} b^{\prime} a a^{\prime}, b b^{\prime} a b^{\prime}\right\} .
$$

It can be easily computed that the code $W$ extends to a partition code in only one fashion:

$$
W \cup\left\{a b b^{\prime} a^{\prime}, a^{\prime} a^{\prime} a^{\prime} b^{\prime}, b b^{\prime} a b, b^{\prime} a b a\right\} .
$$

Similarly, the code $\left\{a b b^{\prime} a^{\prime}, a^{\prime} a^{\prime} a^{\prime} b^{\prime}, b b^{\prime} a b, b^{\prime} a b a\right\}$ can be extended to a partition code which is different from (5.4) in only one fashion:

$$
V \cup\left\{a b b^{\prime} a^{\prime}, a^{\prime} a^{\prime} a^{\prime} b^{\prime}, b b^{\prime} a b, b^{\prime} a b a\right\} .
$$

Thus, in dimension four the pair $V, W$ is the only pair of polybox codes, up to an isomorphism, which are disjoint, equivalent and do not contain a twin pair.

For $d=5,6$, by Claims 25 and 26, every cover of a pair of words

$$
\{v, u\} \in\left\{\left\{b b b b b, b^{\prime} b^{\prime} b^{\prime} b b\right\},\left\{b b b b b, b^{\prime} b^{\prime} b^{\prime} b^{\prime} b^{\prime}\right\},\left\{b b b b b b, b^{\prime} b^{\prime} b^{\prime} b b b\right\},\left\{b b b b b b, b^{\prime} b^{\prime} b^{\prime} b^{\prime} b^{\prime} b\right\}\right\}
$$

by pairwise dichotomous words without twin pairs cannot be completed to a twin pair free polybox code $W$ with twelve words which has all siblings. Therefore, in dimensions five and six we have, by Lemma 19, $V_{A^{c}}=W_{A^{c}}=\left\{r_{A^{c}}\right\}$ for some $r \in S^{d}$, where the codes $V_{A}, W_{A}$ are as in dimension four.

Remark 28. The codes $V$ and $W$ were used by Lagarias and Shor[12, 13] and later on by Mackey[17] to construct the counterexamples to Keller's cube tiling conjecture. In the context of this conjecture one of these codes was given first by Corrádi and Szabó in [3], as an example of the maximum clique in a 4-dimensional Keller graph.

\section{Twin pairs in cube tilings of $\mathbb{R}^{7}$}

From Theorem 15 and 27 we obtain the following

Theorem 29. Let $U \subset S^{7}$ be a partition code. If there are $i \in[7]$ and $l \in S$ such that $\left|U^{i, l}\right| \leqslant 12$, then there is a twin pair in $U$. 
Proof. Without loss of generality we may assume that $i=7$ and $l=a$. Since $\bigcup E\left(U^{7, a} \cup\right.$ $\left.U^{7, a^{\prime}}\right)$ is a 7-cylinder in $(E S)^{7}$, the codes $U_{7^{c}}^{7, a}$ and $U_{7 c^{c}}^{7, a^{\prime}}$ are equivalent, where recall that $U_{7^{c}}^{7, a}=\left(U^{7, a}\right)_{7^{c}}$ and $U_{7^{c}}^{7, a^{\prime}}=\left(U^{7, a^{\prime}}\right)_{7^{c}}$. If $U^{7, a}$ or $U^{7, a^{\prime}}$ contains a twin pair, then clearly $U$ does. Thus, we assume that these two codes do not contain a twin pair. If $U_{7^{c}}^{7, a} \cap U_{7^{c}}^{7, a^{\prime}} \neq \emptyset$ and $v_{7^{c}} \in U_{7^{c}}^{7, a} \cap U_{7^{c}}^{7, a^{\prime}}$, then the words $w \in U^{7, a}$ and $p \in U^{7, a^{\prime}}$ such that $v_{7^{c}}=w_{7^{c}}=p_{7^{c}}$ are a twin pair. Therefore, we may assume that $U_{7^{c}}^{7, a}$ and $U_{7 c}^{7, a^{\prime}}$ are disjoint and do not contain a twin pair. It follows from Theorem 15 that $\left|U^{7, a}\right|=12$, and Theorem 27 precisely describes the structure of the codes $U_{7 c}^{7, a}$ and $U_{7 c^{c}}^{7, a^{\prime}}$. Thus, we may assume that $U_{7^{c}}^{7, a}=W$, $A=\{1,2,3,4\}$ and $r_{A^{c}}=a a$, where $W, A$ and $r$ are as in Theorem 27.

From $U^{7, a}$ we choose four words:

$$
\begin{array}{lllllll}
v=a & a^{\prime} & a^{\prime} & a & a & a & a \\
u=a^{\prime} & a & a^{\prime} & a^{\prime} & a & a & a \\
p=b & a & a^{\prime} & a & a & a & a \\
q=b^{\prime} & a & b^{\prime} & a & a & a & a
\end{array}
$$

Since $U$ is a partition code, there is a set $Q \subset U^{1, b^{\prime}}$ such that $p_{1^{c}} \sqsubseteq Q_{1^{c}}$ and $\breve{w}_{1^{c}} \cap \breve{p}_{1^{c}} \neq \emptyset$ for every $w \in Q$. Clearly, for every $i \in\{2, \ldots, 7\}$ and $w \in Q$ we have $w_{i} \neq p_{i}^{\prime}$, for otherwise $\breve{w}_{1^{c}} \cap \breve{p}_{1^{c}}=\emptyset$ which contradicts the definition of $Q$. Every $w \in Q \backslash\{q\}$ is dichotomous to the words $v, u$ and $q$, and therefore $w_{1}=b^{\prime}, w_{2}=a, w_{3}=b$ and $w_{4}=a$ for every $w \in Q \backslash\{q\}$. Let $P=Q \backslash\{q\}$. Then

$$
a a a \sqsubseteq P_{\{5,6,7\}} .
$$

To show this, suppose on the contrary that it is not so. Then there is a point $\left(x_{5}, x_{6}, x_{7}\right) \in$ $E a \times E a \times E a$ such that $\left(x_{5}, x_{6}, x_{7}\right) \notin \bigcup E\left(P_{\{5,6,7\}}\right)$. Taking $x_{1} \in E b, x_{2} \in E a, x_{3} \in$ $E a^{\prime} \backslash E b^{\prime}$ and $x_{4} \in E a$ we obtain the point $x=\left(x_{1}, \ldots, x_{7}\right)$ which belongs to $\breve{p}$ and $x_{1^{c}} \notin \bigcup E\left(Q_{1^{c}}\right)$. Then, $p_{1^{c}} \nsubseteq Q_{1^{c}}$, a contradiction.

If aaa $\in P_{\{5,6,7\}}$, then the words $b^{\prime}$ abaaaa $\in P$ and $q$ form a twin pair.

Let $a a a \notin P_{\{5,6,7\}}$. If $P_{\{5,6,7\}}$ does not contain a twin pair, then its structure is as in Lemma 9 (the case $d=3$ ):

$$
P_{\{5,6,7\}}=\left\{s_{1} s_{2} s_{3}, s_{1}^{\prime} s_{2}^{\prime} s_{3}^{\prime}, a s_{2}^{\prime} s_{3}, s_{1} a s_{3}^{\prime}, s_{1}^{\prime} s_{2} a\right\},
$$

where $s_{i} \notin\left\{a, a^{\prime}\right\}$ for $i=1,2,3$. But then $b^{\prime} a b a s_{1}^{\prime} s_{2} a \in U^{7, a}$ which is not true because $s_{2} \neq a$. Therefore, by Lemma 9 (the case $d=3$ ), the code $P_{\{5,6,7\}}$ contains a twin pair, and hence the code $P$ contains a twin pair because $w_{1}=b^{\prime}, w_{2}=a, w_{3}=b$ and $w_{4}=a$ for every $w \in P$. Since $P \subset U$, the code $U$ contains a twin pair.

We now prove Theorem 1

Proof of Theorem 1 As it was showed in Section 2.3, the set of boxes $\mathscr{F}_{x}=\left\{\left([0,1)^{7}+t\right) \cap\right.$ $\left.\left([0,1]^{7}+x\right) \neq \emptyset: t \in T\right\}$ is a minimal partition of $[0,1]^{7}+x$. Let $U$ be a partition code such that $\mathscr{F}_{x}$ is an exact realization of $U$ (this code can be obtained in the manner described in Section 2.7). Since $r^{+}(T)=5$, there is $i \in[7]$ such that $U=U^{i, l_{1}} \cup U^{i, l_{1}^{\prime}} \cup \cdots \cup U^{i, l_{5}} \cup U^{i, l_{5}^{\prime}}$ and $\left|U^{i, l_{j}}\right| \leqslant 12$ for some $j \in[5]$ because $|U|=128$. By Theorem 29, there is a twin pair 
in $U$ and consequently there is a twin pair in $\mathscr{F}_{x}$. Then the tiling $[0,1)^{7}+T$ contains a twin pair.

From the result of Debroni et al., [10, Theorem 1.1] and Theorm 1 we obtain the following

Corollary 30. If $[0,1)^{7}+T$ is a counterexample to Keller's conjecture in dimension seven, then $r^{-}(T), r^{+}(T) \in\{3,4\}$.

Proof. Theorem 1.1 in [10] says that every cube tiling $[0,1)^{7}+T$ of $\mathbb{R}^{7}$ such that $r^{+}(T) \geqslant 6$ contains a twin pair, and the result of Debroni et al. says that a cube tiling $[0,1)^{7}+T$ with $r^{-}(T) \leqslant 2$ contains a twin pair. Since, by just proved Theorem 1.1, there is a twin pair in every cube tiling $[0,1)^{7}+T$ of $\mathbb{R}^{7}$ such that $r^{+}(T)=5$, in a counterexample tiling $[0,1)^{7}+T$ to Keller's conjecture it must be $r^{-}(T), r^{+}(T) \in\{3,4\}$.

Theorem 27 can be extended to all dimensions $d \geqslant 4$. Since in the presented paper we did not need this result for $d \geqslant 7$ we give this theorem with only a brief sketch of the proof.

Theorem 31. Let $d \geqslant 4$, and let $V, W \subset S^{d}$ be disjoint sets which are equivalent polybox codes without twin pairs. If $|V|=12$, then, up to an isomorphism,

$$
V_{A}=\left\{a a^{\prime} b b^{\prime}, a b b^{\prime} a, a b^{\prime} b^{\prime} b^{\prime}, a^{\prime} a b^{\prime} b^{\prime}, a^{\prime} a^{\prime} a b^{\prime}, a^{\prime} b b^{\prime} b, b a b b^{\prime}, b b b b, b b^{\prime} a^{\prime} b, b^{\prime} a b a^{\prime}, b^{\prime} a^{\prime} b b, b^{\prime} b^{\prime} b^{\prime} b\right\},
$$

$W_{A}=\left\{a^{\prime} a^{\prime} a^{\prime} b, a^{\prime} b a a^{\prime}, b a a^{\prime} a, a a^{\prime} a^{\prime} a, a^{\prime} a a^{\prime} a^{\prime}, a b b a^{\prime}, b b a a, a b^{\prime} a^{\prime} a^{\prime}, b^{\prime} a b^{\prime} a, b^{\prime} a^{\prime} a a, b^{\prime} b^{\prime} a a^{\prime}, b b^{\prime} a b^{\prime}\right\}$, where $A=\{1,2,3,4\} \subseteq[d]$ and $V_{A^{c}}=W_{A^{c}}=\left\{r_{A^{c}}\right\}$ for some $r \in S^{d}$.

A sketch of the proof. We procced by induction on $d$. By Theorem 27 the theorem is true for $d \in\{4,5,6\}$. Let $d \geqslant 7$ and assume that for every $i \in[d]$ there are two letters $l, s \in S$ such that $V^{i, l}$ and $V^{i, s}$ are non-empty sets. Then, by Theorem 19, we may assume that $V$ has all siblings (by Theorem 21 we assume that $V$ and $W$ are written down in the alphabet $\left.\left\{a, a^{\prime} b, b^{\prime}\right\}\right)$. We now consider a graph $G=(V, \mathscr{E})$ of siblings on $V$. Let $u^{0}, v^{0} \in V$ be such that

$$
d\left(v^{0}\right)+d\left(u^{0}\right)=\max \{d(v)+d(u): v, u \in V \text { and } v, u \text { are adjacent }\} .
$$

If $d\left(v^{0}\right)+d\left(u^{0}\right) \geqslant 10$, then in the similar manner as in the proof of Lemma 16 we show that the polybox code $V$ does not contain all siblings, and then, by Theorem $19,|V|>12$, a contradiction.

If $d\left(v^{0}\right)+d\left(u^{0}\right) \leqslant 9$, then, by Lemma $6, d(G) \leqslant 9 / 2$. Since $d(G)|V|=2|\mathscr{E}|$ and $2|\mathscr{E}| \geqslant 8 d \geqslant 56$ (recall that $|\mathscr{E}| \geqslant 4 d$ ), we have $|V|>12$, which is a contradiction.

Therefore, there is $i \in[d]$ and a letter $l \in S$ such that $V=V^{i, l}$. By the inductive hypothesis, $V_{i^{c}}$ and $W_{i^{c}}$ are of the forms given in the theorem.

A set $V \subset\{0,1,2,3\}^{d}$ is a clique in a $d$-dimensional Keller graph if and only if $V$ is a polybox code without twin pairs (recall that in the alphabet $S=\{0,1,2,3\}$ the complementation is given by $0^{\prime}=2$ and $1^{\prime}=3$ ). Thus, we may speak about equivalent 
cliques in a $d$-dimensional Keller graph ([10]): Cliques $V$ and $W$ in a $d$-dimensional Keller graph are equivalent if $\sum_{v \in V} g(v, w)=2^{d}$ for every $w \in W$ and $\sum_{w \in W} g(w, v)=2^{d}$ for every $v \in V$.

Theorem 31 for cliques in a $d$-dimensional Keller graph reads as follows:

Theorem 32. Let $d \geqslant 4$, and let $V, W \subset\{0,1,2,3\}^{d}$ be two disjoint sets which are equivalent cliques in the d-dimensional Keller graph. If $|V|=12$, then, up to an isomorphism (of polybox codes),

$$
\begin{gathered}
V_{A}=\{0213,0130,0333,2033,2203,2131,1013,1111,1321,3012,3211,3331\}, \\
W_{A}=\{2221,2102,1020,0220,2022,0112,1100,0322,3030,3200,3302,1303\},
\end{gathered}
$$

where $A=\{1,2,3,4\} \subseteq[d]$ and $V_{A^{c}}=W_{A^{c}}=\left\{r_{A^{c}}\right\}$ for some $r \in\{0,1,2,3\}^{d}$.

In [10] we extended the notion of a $d$-dimensional Keller graph: If $S$ is an alphabet with a complementation, then a d-dimensional Keller graph on the set $S^{d}$ is the graph in which two vertices $u, v \in S^{d}$ are adjacent if they are dichotomous but do not form a twin pair.

From Theorem 29 we obtain the following

Corollary 33. Every clique in a 7-dimensional Keller graph on $S^{7}$ which contains at least five vertices $u^{1}, \ldots, u^{5}$ such that $u_{i}^{n} \notin\left\{u_{i}^{m},\left(u_{i}^{m}\right)^{\prime}\right\}$ for some $i \in[7]$ and every $n, m \in$ $\{1, \ldots, 5\}, n \neq m$, has less than $2^{7}$ elements.

Proof. Assume on the contrary that there is a clique $U$ containing vertices $u^{1}, \ldots, u^{5}$ and $|U|=2^{7}$. Thus, $U$ is a partition code without twin pairs. Since $u_{i}^{n} \notin\left\{u_{i}^{m},\left(u_{i}^{m}\right)^{\prime}\right\}$ for every $n, m \in\{1, \ldots, 5\}, n \neq m$, it follows that $\left|U^{i, u_{i}^{m}}\right| \leqslant 12$ for some $m \in[5]$. By Theorem 29, there is a twin pair in $U$, a contradiction.

Remark 34. What next? Our computer experiments made together with our colleague Krzysztof Przesławski show that there is a chance that the case $r^{+}(T)=4$ can be resolve using the same methods as the case $r^{+}(T)=5$, but a scale of the computations will be much more larger than that for $r^{+}(T)=5$. Moreover, to obtain initial configurations for the computations we need some new results on the rigidity of polyboxes.

\section{References}

[1] N. Alon, T. Bohman, R. Holzman and D. J. Kleitman, On partitions of discrete boxes, Discrete Math. 257 (2002), 255-258.

[2] K. Corrádi and S. Szabó, Cube tiling and covering a complete graph, Discrete Math. 85 (1990), 319-321.

[3] K. Corrádi and S. Szabó, A combinatorial approach for Keller's conjecture, Period. Math. Hungar. 21 (1990), 95-100. 
[4] J. Debroni, J.D Eblen, M.A. Langston, W. Myrvold, P. Shor and D. Weerapurage, A complete resolution of the Keller maximum clique problem, Proceedings of the Twenty-Second Annual ACM-SIAM Symposium on Discrete Algorithms, 2011.

[5] J. Grytczuk, A. P. Kisielewicz and K. Przesławski, Minimal Partitions of a Box into Boxes, Combinatorica 24 (2004), 605-614.

[6] G. Hajós, Uber einfache und mehrfache Bedeckung des n-dimensionalen Raumes mit einem Würfelgitter, Math. Z. 47 (1941), 427-467.

[7] O. H. Keller, Über die lückenlose Erfüllung des Raumes mit Würfeln, J. Reine Angew. Math. 163 (1930), 231-248.

[8] A. P. Kisielewicz, On the structure of cube tilings in $\mathbb{R}^{3}$ and $\mathbb{R}^{4}$, J. Combin. Theory Ser. A 120 (2013), 1-10.

[9] A. P. Kisielewicz, Partitions and balanced matchngs if an $n$-dimensional cube, European J. Combin. 40 (2014), 93-107.

[10] A. P. Kisielewicz, Rigid polyboxes and Keller's conjecture, submitted, arXiv:1304.1639v5

[11] A. P. Kisielewicz, K. Przesławski, Polyboxes, cube tilings and rigidity, Discrete Comput. Geom. 40 (2008), 1-30.

[12] J. C. Lagarias and P. W. Shor, Keller's cube-tiling conjecture is false in high dimensions, Bull. Amer. Math. Soc. 27 (1992), 279-287.

[13] J. C. Lagarias and P. W. Shor, Cube tilings of $\mathbb{R}^{d}$ and nonlinear codes, Discrete Comput. Geom. 11 (1994), 359-391.

[14] J. Lawrence, Tiling $\mathbb{R}^{d}$ by translates of orthants, Convexity and Related Comb. Geometry Proc. of the Second Univ. of Oklahoma Conf. (1982), 203-207.

[15] M. Łysakowska and K. Przesławski, Keller's conjecture on the existence of columns in cube tilings of $\mathbb{R}^{n}$, Adv. Geom. 12 (2012), 329-352.

[16] M. Eysakowska and K. Przesławski, On the structure of cube tilings and unextendible systems of cubes in low dimension, European J. Combin. 32 (2011), 14171427.

[17] J. Mackey, A cube tiling of dimension eight with no facesharing, Discrete Comput. Geom. 28 (2002), 275-279.

[18] H. Minkowski, Diophantische Approximationen, Teubner, Leipzig, 1907.

[19] O. Perron, Über lückenlose Ausfüllung des n-dimensionalen Raumes durch kongruente Würfeln, Math. Z. 46 (1940), 1-26.

[20] S. Szabó, A reduction of Keller's conjecture, Period. Math. Hungar. 17 (1986), 265-277. 